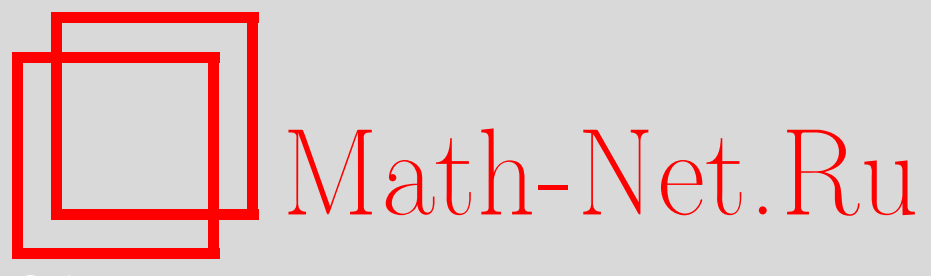

А. А. Гайфуллин, Построение комбинаторных многообразий с заданными наборами линков вершин, Изв. РАН. Сер. матем., 2008, том 72, выпуск 5, 3-62

DOI: https://doi.org/10.4213/im2681

Использование Общероссийского математического портала Math-Net.Ru подразумевает, что вы прочитали и согласны с пользовательским соглашением http://www . mathnet.ru/rus/agreement

Параметры загрузки:

IP : 18.234 .156 .22

26 апреля 2023 г., 17:39:22

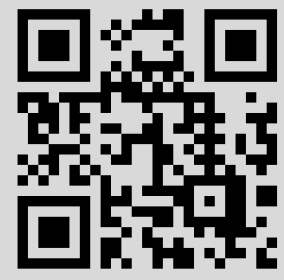




\section{А. А. Гайфуллин \\ Построение комбинаторных многообразий с заданными наборами линков вершин}

Изучается преобразование $\mathcal{L}$, сопоставляющее каждому ориентированному замкнутому комбинаторному многообразию набор классов изоморфизма линков его вершин. Ставится задача об обращении преобразования $\mathcal{L}$. Показано, что эта задача тесно связана с классической проблемой Стинрода о реализации циклов и конструкцией Рохлина-Шварца-Тома комбинаторных классов Понтрягина. Получено условие сбалансированности, являющееся необходимым для того, чтобы набор классов изоморфизма комбинаторных сфер принадлежал образу преобразования $\mathcal{L}$. Дана явная конструкция, показывающая, что каждый набор классов изоморфизма комбинаторных сфер, удовлетворяющий этому условию сбалансированности, попадает в образ преобразования $\mathcal{L}$ после перехода к кратному набору и добавления некоторого количества пар вида $(Z,-Z)$, где $-Z$ есть сфера $Z$ с обращенной ориентацией. Эта конструкция позволяет по каждому сингулярному симплициальному циклу $\xi$ пространства $X$ явно построить комбинаторное многообразие $M$ и отображение $\varphi: M \rightarrow X$ такие, что $\varphi_{*}[M]=r[\xi]$ для некоторого натурального числа $r$. Построение проведено с помощью разрешения особенностей цикла $\xi$. Даны приложения основной конструкции к изучению кобордизмов многообразий с особенностями и кобордизмов простых клеток. В частности, доказано существование локальных формул для всех рациональных аддитивных инвариантов кобордизмов с особенностями. В качестве приложения построены явные, хотя и неэффективные, локальные комбинаторные формулы для полиномов от рациональных классов Понтрягина комбинаторных многообразий.

Библиография: 38 наименований.

\section{§1. Введение}

Комбинаторной сферой называется симплициальный комплекс, кусочно линейно гомеоморфный границе симплекса; комбинаторным многообразием называется симплициальный комплекс, линк каждой вершины которого является комбинаторной сферой. Все рассматриваемые многообразия предполагаются замкнутыми. Изоморфизмом ориентированных комбинаторных многообразий мы называем симплициальное отображение, имеющее симплициальное обратное и сохраняющее ориентацию.

Каждой триангуляции многообразия можно сопоставлять различные характеризующие ее комбинаторные данные. Простейшим примером таких данных является $f$-вектор $\left(f_{0}, f_{1}, \ldots, f_{n}\right)$, где через $f_{i}$ обозначено количество $i$-мерных

Работа выполнена при финансовой поддержке РФФИ (грант № 08-01-91855-КО-а) и Программы Президента РФ "Поддержка ведущих научных школ" (грант НШ-1824.2008.1).

(C) А. А. ГАйФуллин, 2008 
симплексов в триангуляции. В более сложных случаях комбинаторные данные тем или иным образом описывают взаимное расположение симплексов. Некоторые функции от комбинаторных данных дают инварианты многообразия, не зависящие от триангуляции. Например, эйлерова характеристика многообразия выражается через его $f$-вектор. В настоящей статье мы будем сопоставлять каждому ориентированному комбинаторному многообразию неупорядоченный набор классов изоморфизма линков его вершин. Интерес к таким комбинаторным данным обусловлен в том числе тем, что числа Понтрягина многообразия могут быть вычислены по набору классов изоморфизма линков его вершин. Существование функций, вычисляющих числа Понтрягина по набору классов изоморфизма линков, следует из результата Н. Левитта и К. Рурка [1] (см. также [2], [3]). Вопрос о явных формулах будет обсуждаться ниже.

Таким образом, основным объектом изучения в настоящей статье является преобразование $\mathcal{L}$, сопоставляющее каждому ориентированному комбинаторному многообразию $K$ неупорядоченный набор классов изоморфизма линков его вершин. Линки вершин многообразия $K$ наделяются индуцированными ориентациями. Таким образом, набор классов изоморфизма линков вершин - это набор классов изоморфизма ориентированных комбинаторных сфер. В дальнейшем мы часто не будем различать изоморфные комбинаторные сферы. Мы будем обозначать класс изоморфизма комбинаторной сферы так же, как саму эту сферу, и, допуская вольность речи, будем говорить, что преобразование $\mathcal{L}$ сопоставляет каждому ориентированному комбинаторному многообразию набор линков его вершин.

Изучая преобразование $\mathcal{L}$, поставим задачу о его обращении.

Вопрос 1.1. Для каких наборов $\left(Y_{1}, Y_{2}, \ldots, Y_{k}\right)$ ориентированных $(n-1)$ мерных комбинаторных сбер существует ориентированное $n$-мерное комбинаторное многообразие, набор линков вершин которого совпадает с точностью до изоморбизма с набором $\left(Y_{1}, Y_{2}, \ldots, Y_{k}\right)$ ?

Рассмотрим свободную абелеву группу, порожденную классами изоморфизма ориентированных $(n-1)$-мерных комбинаторных сфер. Профакторизуем эту группу по соотношениям $Y+(-Y)=0$, где через $-Y$ обозначена комбинаторная сфера, получающаяся из $Y$ заменой ориентации на противоположную. Полученную абелеву группу мы будем обозначать через $\mathcal{T}_{n}$. Формула

$$
d Y=\sum \operatorname{link} y
$$

где суммирование ведется по всем вершинам $y$ комбинаторной сферы $Y$, определяет дифференциал $d: \mathcal{T}_{n} \rightarrow \mathcal{T}_{n-1}$, превращающий градуированную группу $\mathcal{T}_{*}$ в цепной комплекс. Комплекс $\mathcal{T}_{*}$ был впервые определен автором в [2].

Ввиду наличия групповой структуры в множестве $\mathcal{T}_{n}$ нам будет удобнее работать не с преобразованием $\mathcal{L}$, а с преобразованием $\mathcal{L}_{\mathcal{T}}$, сопоставляющим каждому ориентированному $n$-мерному комбинаторному многообразию сумму линков его вершин в группе $\mathcal{T}_{n}$. Еще одно преимущество преобразования $\mathcal{L}_{\mathcal{T}}$ состоит в том, что оно коммутирует с операцией взятия барицентрического подразделения с точностью до элементов порядка 2. Задача об обращении преобразования $\mathcal{L}_{\mathcal{T}}$ формулируется следующим образом. 
Вопрос 1.2. Для каких наборов $\left(Y_{1}, Y_{2}, \ldots, Y_{k}\right)$ ориентированных $(n-1)$ мерных комбинаторных сфер существует ориентированное $n$-мерное комбинаторное многообразие, набор линков вершин которого совпадает с точностью до изоморбизма с набором

$$
\left(Y_{1}, Y_{2}, \ldots, Y_{k}, Z_{1}, Z_{2}, \ldots, Z_{l},-Z_{1},-Z_{2}, \ldots,-Z_{l}\right)
$$

для некоторого набора $\left(Z_{1}, Z_{2}, \ldots, Z_{l}\right)$ ориентированных $(n-1)$-мерных комбинаторных сфер?

НЕОБХодимое условиЕ. Для того чтобы ответ на вопрос 1.2 (и, тем более, на вопрос 1.1) был утвердительным, необходимо, чтобы вершины несвязного объединения $Y_{1} \sqcup Y_{2} \sqcup \cdots \sqcup Y_{k}$ могли быть разбиты на пары так, что линки вершин в каждой паре изоморфны с изменением ориентации.

Наборы ориентированных комбинаторных сфер, удовлетворяющие этому необходимому условию, мы будем называть сбалансированными. Очевидно, что сбалансированные наборы комбинаторных сфер - это в точности циклы комплекса $\mathcal{T}_{*}$.

Основным результатом настоящей статьи является явная конструкция, дающая следующий частичный ответ на вопрос 1.2 .

Теорема 1.1. Пусть $\left(Y_{1}, Y_{2}, \ldots, Y_{k}\right)$ - сбалансированный набор ориентированных $(n-1)$-мерных комбинаторных сфер. Тогда существует ориентированное $n$-мерное комбинаторное многообразие $K$, набор линков вершин которого совпадает с точностью до изоморфизма с набором

$$
(\underbrace{Y_{1}, \ldots, Y_{1}}_{r}, \underbrace{Y_{2}, \ldots, Y_{2}}_{r}, \ldots, \underbrace{Y_{k}, \ldots, Y_{k}}_{r}, Z_{1}, Z_{2}, \ldots, Z_{l},-Z_{1},-Z_{2}, \ldots,-Z_{l})
$$

для некоторого натурального числа $r$ и некоторых ориентированных $(n-1)$ мерных комбинаторных сфер $Z_{1}, Z_{2}, \ldots, Z_{l}$.

Вопросы 1.1 и 1.2 можно ставить не только для триангуляций, но и для кубических разбиений многообразий, а также для более широкого класса кубически клеточных разбиений многообразий. Кубически клеточный комплекс (или кубически клеточное разбиение) - это разбиение на кубы, в котором два куба могут пересекаться не только по одной грани, но и по целому подкомплексу их границы. Линк вершины в кубически клеточном комплексе определяется как симплициально клеточный комплекс, высекаемый кубами исходного комплекса на малой сфере с центром в рассматриваемой вершине. Кубически клеточным комбинаторным многообразием мы называем кубически клеточный комплекс, линк каждой вершины которого кусочно линейно гомеоморфен границе симплекса. (Строгие определения будут даны в п. 2.1.)

Для кубически клеточных комбинаторных многообразий мы получаем следующий частичный ответ на вопрос 1.1 .

ТЕОРема 1.2. Пусть $\left(Y_{1}, Y_{2}, \ldots, Y_{k}\right)$ - сбалансированный набор ориентированных $(n-1)$-мерных комбинаторных сфер. Тогда существует ориентированное $n$-мерное кубически клеточное комбинаторное многообразие $X$, набор 
линков вершин которого совпадает с точностью до изоморфизма с набором

$$
(\underbrace{Y_{1}^{\prime}, \ldots, Y_{1}^{\prime}}_{r}, \underbrace{Y_{2}^{\prime}, \ldots, Y_{2}^{\prime}}_{r}, \ldots, \underbrace{Y_{k}^{\prime}, \ldots, Y_{k}^{\prime}}_{r})
$$

для некоторого натурального числа $r$. Здесь $Y_{i}^{\prime}$ - первое барицентрическое подразделение комбинаторной сферь $Y_{i}$.

Явному построению такого кубически клеточного комбинаторного многообразия $X$ посвящена бо́льшая часть $\S 2$. В дальнейшем мы будем ссылаться на эту конструкцию как на основную конструкцию настоящей работы. В пп. 2.7, 2.8 мы, используя основную конструкцию, даем явную конструкцию комбинаторного многообразия $K$ из теоремы 1.1 .

При построении комплексов $X$ и $K$ мы нигде не используем условие, что комплексы $Y_{i}$ являются комбинаторными сферами. Вместо сбалансированного набора комбинаторных сфер можно рассматривать произвольный сбалансированный набор нормальных псевдомногообразий (определение см. в п. 2.1). Конечно, в этом случае комплексы $X$ и $K$ будут не комбинаторными многообразиями, а лишь нормальными псевдомногообразиями. В $\S 2$ мы описываем основную конструкцию в максимальной общности, т. е. для нормальных псевдомногообразий. Формулировки аналогов теорем 1.1 и 1.2 в этом случае приведены в п. 2.2.

Пусть $Y_{1}, Y_{2}, \ldots, Y_{k}$ - сбалансированный набор комбинаторных сфер, причем задано конкретное разбиение множества вершин комбинаторных сфер $Y_{1}, Y_{2}, \ldots, Y_{k}$ на пары и конкретные изменяющие ориентацию изоморфизмы линков вершин в каждой паре. Тогда теорема 1.2 может быть усилена: кубически клеточное многообразие $X$ может быть выбрано согласованно с заданными разбиением на пары и изоморфизмами (см. теорему 2.3).

В п. 2.9 мы устанавливаем связь нашей основной конструкции с конструкцией мальх накрытий, принадлежащей М. Дэвису и Т. Янушкевичу [4] (см. также [5]).

ЗАМЕчАНИЕ 1.1. Вопросы 1.1 и 1.2 являются примерами часто встречающейся в топологии проблемы характеризации наборов локальных данных, которые могут быть реализованы как локальные инварианты некоторого глобального объекта. Другим примером такой задачи является задача о соотношениях между классами кобордизмов циклов, реализующих классы Понтрягина стабильно комплексного многообразия, решенная В. М. Бухштабером и А. П. Веселовым [6]. Еще один пример проблемы такого типа - проблема характеризации возможных наборов локальных весов действия группы $\mathbb{Z}_{p}$ с изолированными неподвижными точками на замкнутом стабильно комплексном многообразии (см., например, [7]).

В $\S 3-5$ приведены приложения основной конструкции.

В 33 мы изучаем кобордизмы многообразий с особенностями. Под многообразием с особенностями из класса $\mathrm{C}$ мы понимаем псевдомногообразие, линки всех симплексов которого принадлежат классу С. В качестве С можно брать 
произвольный класс псевдомногообразий, удовлетворяющий некоторым естественным аксиомам. Отметим, что группы кобордизмов многообразий с особенностями, определенные с помощью нашей конструкции, не совпадают с группами, определенными с помощью классической конструкции Сулливана-Бааса (см. [8]-[10]). Подробнее связь этих двух конструкций обсуждается в п. 3.5. Рассматривая класс С вместо класса комбинаторных сфер, можно определить аналог цепного комплекса $\mathcal{T}_{*}$, который мы обозначаем через $\mathcal{T}_{*}^{\mathrm{C}}$. Мы применяем конструкцию из $\S 2$ для изучения связи между группами кобордизмов ориентированных многообразий с особенностями из класса С и группами гомологий комплекса $\mathcal{T}_{*}^{\mathrm{C}}$. Основным результатом $\S 3$ является утверждение о том, что эти группы изоморфны по модулю класса групп кручения. В п. 3.4 мы изучаем двойственный к $\mathcal{T}_{*}^{\mathrm{C}}$ коцепной комплекс $\mathcal{T}_{\mathrm{C}}^{*}(A)=\operatorname{Hom}\left(\mathcal{T}_{*}^{\mathrm{C}}, A\right)$, где $A-$ абелева группа. Коциклы этого комплекса задают локальные формулы для $A$-значных аддитивных инвариантов кобордизма ориентированных многообразий с особенностями из класса С. Мы доказываем, что для любого $\mathbb{Q}$-значного инварианта кобордизмов многообразий с особенностями существует локальная формула, единственная с точностью до кограницы комплекса $\mathcal{T}_{\mathrm{C}}^{*}(\mathbb{Q})$. В частности, мы получаем более прямое и простое доказательство теоремы автора [2] о том, что градуированная группа когомологий комплекса $\mathcal{T}^{*}(\mathbb{Q})=\operatorname{Hom}\left(\mathcal{T}_{*}, \mathbb{Q}\right)$ аддитивно изоморфна кольцу полиномов от классов Понтрягина с рациональными коэффициентами.

В $\S 4$ основная конструкция применяется к задаче о разрешении особенностей псевдомногообразий и к проблеме Стинрода о реализации классов гомологий посредством образов фундаментальных циклов многообразий. Подход к проблеме Стинрода, основанный на разрешении особенностей циклов, был предложен Д. Сулливаном [11]. Пусть $Z$ - псевдомногообразие, $\Sigma \subset Z$ - подмножество такое, что $Z \backslash \Sigma$ - ориентированное многообразие. Согласно [11] разрешение особенностей псевдомногообразия $Z$ - это отображение $g: M \rightarrow Z$ (где $M-$ многообразие) такое, что ограничение

$$
\left.g\right|_{g^{-1}(Z \backslash \Sigma)}: g^{-1}(Z \backslash \Sigma) \rightarrow Z \backslash \Sigma
$$

является диффеоморфизмом (или кусочно гладким гомеоморфизмом - в зависимости от рассматриваемой категории многообразий). Первым примером псевдомногообразия, не допускающего разрешения особенностей, является 7-мерный цикл, представляющий построенный Р. Томом [12] 7-мерный целочисленный класс гомологий, не реализуемый по Стинроду.

Раздутием nары $(\underset{\widetilde{Z}}{Z}, \Sigma)$ Д. Сулливан называет отображение $g:(\widetilde{Z}, \widetilde{\Sigma}) \rightarrow$ $(Z, \Sigma)$ такое, что $\widetilde{Z} \backslash \widetilde{\Sigma}-$ многообразие и $\left.g\right|_{g^{-1}(Z \backslash \Sigma)}-$ диффеоморфизм (соответственно, кусочно гладкий гомеоморфизм). В [11] он построил полное препятствие $\mathfrak{v}_{s} \in H_{s}\left(Z ; \Omega_{n-s-1}\right)$ к существованию раздутия $(\widetilde{Z}, \widetilde{\Sigma})$ пары $(Z, \Sigma)$ такого, что $\operatorname{dim} \widetilde{\Sigma}<\operatorname{dim} \Sigma\left(\right.$ см. также [13]). Здесь $s=\operatorname{dim} \Sigma, n=\operatorname{dim} Z, \Omega_{q}-$ группа кобордизмов гладких (соответственно, кусочно линейных) ориентированных $q$-мерных многообразий. Если все препятствия Сулливана последовательно оказываются нулевыми, мы можем, уменьшая размерность множества $\Sigma$, последовательно разрешить особенности псевдомногообразия $Z$. Еще Д. Сулливан заметил, что эти препятствия дают геометрическую интерпретацию дифференциалов спектральной последовательности Атья-Хирцебруха 
в теории гладких (соответственно, кусочно линейных) бордизмов. Действительно, фундаментальный класс $[Z] \in H_{n}(Z ; \mathbb{Z})=H_{n}\left(Z ; \Omega_{0}\right)$ является циклом дифференциалов $d_{2}, \ldots, d_{s-1}$ и $d_{s}([Z])=\mathfrak{v}_{s}$. Следовательно, препятствия Сулливана являются элементами конечного порядка. Неулучшаемые оценки порядков дифференциалов спектральной последовательности Атья-Хирцебруха в теории гладких ориентированных бордизмов были получены В. М. Бухштабером [14]. В [15] С. Буонкристиано и М. Дедо непосредственно геометрически оценили порядки препятствий Сулливана. Эти оценки гораздо слабее, чем оценки В.М. Бухштабера. Отметим, что в случае, когда препятствия к разрешению особенностей равны нулю, результаты работ [11], [13], [15] не дают явной конструкции многообразия $M$ и отображения $g: M \rightarrow Z$, разрешающего особенности.

Мы используем более общее понятие разрешения особенностей, которое можно назвать разрешением особенностей с кратностями. Разрешением особенностей псевдомногообразия $Z$ с кратностью $r$ мы будем называть кусочно гладкое отображение $g: M \rightarrow Z$ (где $M$ - кусочно линейное многообразие) такое, что ограничение

$$
\left.g\right|_{g^{-1}(Z \backslash \Sigma)}: g^{-1}(Z \backslash \Sigma) \rightarrow Z \backslash \Sigma
$$

является $r$-листным накрытием. Из конечности порядков препятствий Сулливана несложно выводится, что разрешение особенностей с некоторой кратностью возможно всегда. Основным результатом $\S 4$ является явная комбинаторная конструкция, сопоставляющая каждому ориентированному псевдомногообразию $Z$, разбитому на простые клетки, комбинаторное многообразие $M$ и отображение $g: M \rightarrow Z$, являющееся разрешением особенностей с некоторой кратностью $r$. При этом в качестве множества $\Sigma$ берется остов коразмерности 2 разбиения $Z$. (Определение разбиения на простые клетки см. в п. 4.1; в частности, любая триангуляция является разбиением на простые клетки.)

При разрешении особенностей одного и того же ориентированного $n$-мерного псевдомногообразия могут получаться комбинаторные многообразия, представляющие разные классы в группе ориентированных кусочно линейных кобордизмов $\Omega_{n}^{\mathrm{SPL}}$. Такое же явление имеет место при разрешении особенностей алгебраических многообразий по Хиронаке. Тем не менее, оказывается, что наша конструкция из $\S 4$ приводит к вполне определенному классу

$$
\frac{[M]}{r} \in \Omega_{n}^{\mathrm{SPL}} \otimes \mathbb{Q}=\Omega_{n}^{\mathrm{SO}} \otimes \mathbb{Q},
$$

зависящему лишь от разбиения псевдомногообразия $Z$ на простые клетки (см. п. 4.5). В частности, если $Z$ - симплициальное или кубическое разбиение, многообразие $M$ будет представлять нулевой класс в группе $\Omega_{n}^{\mathrm{SPL}} \otimes \mathbb{Q}$.

Пусть теперь $R$ - топологическое пространство, $x \in H_{n}(R ; \mathbb{Z})$. Класс гомологий $x$ всегда может быть реализован как образ фундаментального класса некоторого ориентированного псевдомногообразия $Z$. Применив к псевдомногообразию $Z$ нашу конструкцию разрешения особенностей с кратностью $r$, мы получим ориентированное кусочно линейное многообразие $M$ и отображение

$$
\varphi: M \stackrel{g}{\longrightarrow} Z \longrightarrow R,
$$

реализующее класс гомологий $r x$. 
Таким образом, мы получаем чисто комбинаторное конструктивное доказательство того, что каждый целочисленный класс гомологий $x \in H_{n}(R ; \mathbb{Z})$ с некоторой кратностью $r$ может быть реализован образом фундаментального класса кусочно линейного многообразия. Это доказательство не использует теорем трансверсальности и каких бы то ни было алгебро-топологических результатов. K сожалению, при таком комбинаторном подходе нам не удается проследить за числом $r$ : оно у нас будет существенно зависеть от комбинаторики клеточного разбиения $Z$, реализующего класс гомологий $x$, и может быть сколь угодно большим при фиксированном $n$.

Интересно поставить вопрос об описании класса бордизмов

$$
\frac{[\varphi]}{r} \in \Omega_{n}^{\mathrm{SPL}}(R) \otimes \mathbb{Q}=\Omega_{n}^{\mathrm{SO}}(R) \otimes \mathbb{Q} .
$$

Содержательный ответ получается в двух случаях:

1) $Z$ - симплициальное псевдомногообразие, т. е. $Z \rightarrow R$ - сингулярный симплициальный цикл (см. п. 4.6);

2) $R$ - комбинаторное многообразие и отображение $Z \rightarrow R$ задает клеточный цикл в двойственном разбиении $R^{*}$ (см. п. 4.7).

В каждом из этих случаев класс $\frac{[\varphi]}{r}$ зависит только от класса гомологий $x$.

Если отображение $Z \rightarrow R$ задает сингулярный симплициальный цикл, то многообразие $M$ имеет нулевые рациональные классы Понтрягина. Поэтому класс $\frac{[\varphi]}{r}$ является образом класса гомологий $x$ при отображении

$$
H_{*}(R ; \mathbb{Z}) \stackrel{\eta}{\longrightarrow} H_{*}\left(R ; \Omega_{*}^{\mathrm{SPL}} \otimes \mathbb{Q}\right) \stackrel{\left(\mathrm{ch}^{\mathrm{SPL}}\right)^{-1}}{\longrightarrow} \Omega_{*}^{\mathrm{SPL}}(R) \otimes \mathbb{Q}
$$

где $\eta$ - гомоморфизм, индуцированный вложением $\mathbb{Z}=\Omega_{0}^{\mathrm{SPL}} \subset \Omega_{0}^{\mathrm{SPL}} \otimes \mathbb{Q}$, и

$$
\operatorname{ch}^{\mathrm{SPL}}: \Omega_{*}^{\mathrm{SPL}}(R) \rightarrow H_{*}\left(R ; \Omega_{*}^{\mathrm{SPL}} \otimes \mathbb{Q}\right)
$$

- характер Чженя-Дольда в теории ориентированных кусочно линейных бордизмов.

Если отображение $Z \rightarrow R$ задает клеточный цикл в разбиении $R^{*}$, двойственном комбинаторному многообразию $R$, то рациональные классы Понтрягина многообразия $M$ совпадают с образами рациональных классов Понтрягина многообразия $R$ при отображении $\varphi^{*}$. Поэтому класс $\frac{[\varphi]}{r}$ является образом класса гомологий $x$ при отображении

$$
\begin{aligned}
H_{*}(R ; \mathbb{Z}) \stackrel{D}{\longrightarrow} H^{*}(R ; \mathbb{Q}) \stackrel{\eta}{\longrightarrow} H^{*}\left(R ; \Omega_{\mathrm{SPL}}^{*} \otimes \mathbb{Q}\right) \stackrel{\mathrm{ch}_{\mathrm{SPL}}^{-1}}{\longrightarrow} \\
\stackrel{\mathrm{ch}_{\mathrm{SPL}}^{-1}}{\longrightarrow} \Omega_{\mathrm{SPL}}^{*}(R) \otimes \mathbb{Q} \stackrel{D_{\mathrm{SPL}}^{-1} \otimes \mathbb{Q}}{\longrightarrow} \Omega_{*}^{\mathrm{SPL}}(R) \otimes \mathbb{Q},
\end{aligned}
$$

где $\eta$ - гомоморфизм, индуцированный вложением $\mathbb{Z}=\Omega_{\mathrm{SPL}}^{0} \subset \Omega_{\mathrm{SPL}}^{0} \otimes \mathbb{Q}$, $D$ и $D_{\mathrm{SPL}}$ - операторы двойственности Пуанкаре в когомологиях и в ориентированных кусочно линейных кобордизмах соответственно и

$$
\operatorname{ch}_{\mathrm{SPL}}: \Omega_{\mathrm{SPL}}^{*}(R) \rightarrow H^{*}\left(R ; \Omega_{\mathrm{SPL}}^{*} \otimes \mathbb{Q}\right)
$$


- характер Чженя-Дольда в теории ориентированных кусочно линейных кобордизмов.

В п. 4.2 мы изучаем кольцо $\mathcal{P}_{*}$ кобордизмов ориентированных простых клеток. Простая клетка - это клетка, двойственная комбинаторной сфере (строгое определение см. в п. 4.1). В частности, простыми клетками являются простые многогранники. Границей ориентированной простой клетки называется формальная сумма ее гиперграней, взятых с индуцированными ориентациями. Таким образом, кобордизмами простых клеток являются простые клетки на единицу большей размерности. Существует канонический гомоморфизм $\Omega_{*}^{\mathrm{SPL}} \rightarrow \mathcal{P}_{*}$, где $\Omega_{*}^{\mathrm{SPL}}-$ кольцо ориентированных кусочно линейных кобордизмов. Вопрос о строении этого гомоморфизма тесно связан с вопросом 1.2. В частности, из теоремы 1.1 следует, что этот гомоморфизм является изоморфизмом по модулю класса групп кручения.

Еще одним приложением основной конструкции из 2 является построение явных локальных комбинаторных формул для рациональных классов Понтрягина комбинаторных многообразий (см. § 5). Задача формулируется следующим образом: по заданному комбинаторному многообразию построить явно симплициальный цикл, класс гомологий которого двойствен по Пуанкаре заданному рациональному классу Понтрягина (или полиному от классов Понтрягина) этого многообразия. Слово “явно" означает, что мы должны предъявить алгоритм, который вычисляет искомый цикл только по комбинаторному строению заданного многообразия. Первая комбинаторная формула для первого рационального класса Понтрягина была получена А. М. Габриэловым, И. М. Гельфандом и М. В. Лосиком в 1975 г. в работе [16]. Немного позже этот результат был улучшен Р. МакФерсоном [17]. Наиболее простая и эффективная формула для первого класса Понтрягина была получена автором [2] в 2004 г. Для старших классов Понтрягина известно два подхода к построению комбинаторных формул. Один из них принадлежит И. М. Гельфанду и Р. МакФерсону [18], другой - Дж. Чигеру [19]. К сожалению, ни один из этих подходов не приводит к полностью комбинаторной формуле, т. е. такой формуле, которая могла бы быть применена для произвольного комбинаторного многообразия без каких бы то ни было дополнительных структур и давала бы явный комбинаторный алгоритм для вычисления искомого цикла. Еще один подход к задаче комбинаторного вычисления классов Понтрягина, развивающий идеи М. Громова, принадлежит А. С. Мищенко. В работе [20] он построил локальную комбинаторную формулу Хирцебруха, что позволило ему дать локальное определение рациональных классов Понтрягина кусочно линейного многообразия. Однако получить на этом пути явную формулу, вычисляющую характеристический цикл по триангуляции многообразия, пока никому не удалось. Сравнению различных формул для классов Понтрягина триангулированных многообразий посвящен обзор автора [3].

В настоящей статье мы строим комбинаторные формулы для всех полиномов от рациональных классов Понтрягина. К сожалению, полученные формулы являются очень неэффективными. Это связано со сложностью основной конструкции из $\S 2$. Тем не менее, полученные формулы являются первыми формулами для старших классов Понтрягина, которые могут быть применены для произвольного комбинаторного многообразия без каких-либо дополнительных 
структур, и дают комбинаторный алгоритм для вычислений. Подход, который мы применяем, отличен от подхода, использованного в [2]. Заметим, что для первого класса Понтрягина формула, построенная в [2], намного проще, чем формула, которую мы строим в данной статье.

Следуя [1]-[3], в настоящей работе мы предполагаем, что искомый характеристический цикл комбинаторного многообразия $K$ задается универсальной локальной формулой вида

$$
f_{\sharp}(K)=\sum_{\substack{\sigma-\operatorname{cuмплекс~} K \\ \text { codim } \sigma=n}} f(\operatorname{link} \sigma) \sigma,
$$

где $f$ - выбранная функция на классах изоморфизма ориентированных $(n-1)$ мерных комбинаторных сфер, меняющая знак при изменении ориентации комбинаторной сферы. Свойство универсальности заключается в том, что функция $f$ не зависит от комбинаторного многообразия $K$, а цепь $f_{\sharp}(K)$ является циклом для любого комбинаторного многообразия $K$. Базисный результат заключается в том, что для любого полинома от рациональных классов Понтрягина существует формула указанного вида. Этот результат был получен автором в [2] и представляет собой усиление результата Н. Левитта и К. Рурка [1]. Более того, в [2] доказано, что локальные формулы для полиномов от рациональных классов Понтрягина - это в точности коциклы комплекса $\mathcal{T}^{*}(\mathbb{Q})$ и локальная формула для каждого полинома от классов Понтрягина определена однозначно с точностью до кограницы комплекса $\mathcal{T}^{*}(\mathbb{Q})$. Таким образом, наша задача сводится к тому, чтобы для каждого полинома от классов Понтрягина научиться явно вычислять значение $f(Y)$ соответствующей функции $f$ по заданной комбинаторной сфере $Y$.

Определение рациональных классов Понтрягина кусочно линейных многообразий было дано в конце 1950-х годов В. А. Рохлиным, А. С. Шварцем [21] и, независимо, Р. Томом [22] (см. также [23]). Согласно их конструкции рациональные классы Понтрягина ориентированного кусочно линейного многообразия $K$ однозначно характеризуются системой всевозможных уравнений вида

$$
\left\langle\pi^{*} L_{l}\left(p_{1}(K), p_{2}(K), \ldots, p_{l}(K)\right),[M]\right\rangle=\operatorname{sign} M,
$$

где $M \subset K \times S^{q}$ - ориентированное $4 l$-мерное подмногообразие с тривиальным нормальным расслоением, $\pi: K \times S^{q} \rightarrow K$ - проекция на первый сомножитель, $L_{l}$ есть $l$-й многочлен Хирцебруха, $\operatorname{sign} M-$ сигнатура многообразия $M$. Тот факт, что это условие полностью определяет классы Хирцебруха

$$
L_{l}(K)=L_{l}\left(p_{1}(K), p_{2}(K), \ldots, p_{l}(K)\right)
$$

и, следовательно, рациональные классы Понтрягина $p_{l}(K)$, вытекает из классической теоремы Р. Тома [12], утверждающей, что каждый целочисленный класс гомологий $x \in H_{4 l}(K ; \mathbb{Z})$ после домножения на некоторое натуральное число $r$ представляется в виде $\pi_{*}[M]$, где $M \subset K \times S^{q}$ - ориентированное подмногообразие с тривиальным нормальным расслоением. "Неявность" конструкции Рохлина-Шварца-Тома заключается в отсутствии явной комбинаторной конструкции такого подмногообразия $M$. Наш подход основан на том, что вместо вложения $M \subset K \times S^{q}$ с тривиальным нормальным расслоением достаточно 
построить отображение $\varphi: M \rightarrow K$ такое, что гомоморфизм $\varphi^{*}$ переводит рациональные классы Понтрягина многообразия $K$ в рациональные классы Понтрягина многообразия $M$. Такие многообразие $M$ и отображение $\varphi$ могут быть явно построены с помощью конструкции из п. 4.7.

Основной результат о локальных формулах для $L$-классов Хирцебруха заключается в том, что функция $f: \mathcal{T}_{4 l} \rightarrow \mathbb{Q}$ такая, что $f(-Y)=-f(Y)$, является локальной формулой для класса $L_{l}$ тогда и только тогда, когда она удовлетворяет системе линейных уравнений

$$
f\left(Y_{1}\right)+f\left(Y_{2}\right)+\cdots+f\left(Y_{k}\right)=\frac{\operatorname{sign} X}{r},
$$

где $\left(Y_{1}, Y_{2}, \ldots, Y_{k}\right)$ - сбалансированный набор $(4 l-1)$-мерных комбинаторных cфер, $X$ и $r$ - кубически клеточное комбинаторное многообразие и натуральное число, полученные с помощью основной конструкции из $\S 2$, примененной к набору $\left(Y_{1}, Y_{2}, \ldots, Y_{k}\right)$. Сигнатура многообразия $X$ может быть явно вычислена либо непосредственно, либо по формуле Раницкого-Сулливана (см. [24], а также п. 5.1). Таким образом, система (1.1) дает явное комбинаторное описание всех локальных формул для $l$-го полинома Хирцебруха. Выбор канонической локальной формулы, т. е. канонического решения $f_{0}$ системы $(1.1)$, описан в п. 5.2 .

В пп. 5.3-5.8 проведено комбинаторное построение умножения в группе коциклов комплекса $\mathcal{T}^{*}(\mathbb{Q})$. Это умножение позволяет по известным локальным формулам для рациональных характеристических классов получить локальную формулу для их произведения, что сразу дает явные локальные формулы для всех полиномов от рациональных классов Понтрягина, так как $L$-классы Хирцебруха порождают кольцо $\mathbb{Q}\left[p_{1}, p_{2}, \ldots\right]$. Однако построенное умножение не является ни билинейным, ни ассоциативным, ни коммутативным, не удовлетворяет формуле Лейбница и, по-видимому, не имеет никакого естественного продолжения на весь комплекс $\mathcal{T}^{*}(\mathbb{Q})$. Вопрос о существовании в комплексе $\mathcal{T}^{*}(\mathbb{Q})$ билинейного ассоциативного умножения, удовлетворяющего формуле Лейбница, остается открытым.

Я хочу выразить глубокую признательность В. М. Бухштаберу за постановки задач и постоянное внимание к моей работе.

\section{§ 2. Основная конструкция}

2.1. Основные определения. Нам будет удобно использовать следующее определение симплициального комплекса.

ОПРЕДЕЛЕНИЕ 2.1. Конечным симплициальным комплексом называется факторпространство несвязного объединения конечного количества замкнутых симплексов $\Delta_{1}, \Delta_{2}, \ldots, \Delta_{q}$ по отношению эквивалентности $\sim$ такому, что:

1) отношение эквивалентности $\sim$ не отождествляет никакие две различные точки одного симплекса $\Delta_{i}$;

2 ) ограничение отношения эквивалентности $\sim$ на каждое несвязное объединение $\Delta_{i} \sqcup \Delta_{j}, i \neq j$, либо пусто, либо совпадает с отождествлением вдоль линейного гомеоморфизма некоторой грани $F_{1} \subset \Delta_{i}$ на некоторую грань $F_{2} \subset \Delta_{j}$.

Образы граней симплексов $\Delta_{i}$ при такой факторизации называются $\kappa л e m$ ками или симплексами этого симплициального комплекса. 
Пересечение любых двух симплексов симплициального комплекса либо пусто, либо является гранью каждого из них. Если два симплекса имеют несколько общих граней, мы придем к более общему понятию симплициалъно клеточного комплекса. Строгое определение симплициально клеточного комплекса получается, если в определении 2.1 заменить условие 2) на следующее условие:

$2^{\prime}$ ) если отношение эквивалентности $\sim$ отождествляет точку $x_{1} \in \Delta_{i}$ с точкой $x_{2} \in \Delta_{j}$, то оно отождествляет некоторую грань $F_{1} \subset \Delta_{i}$, содержащую точку $x_{1}$, с некоторой гранью $F_{2} \subset \Delta_{j}$, содержащей точку $x_{2}$, вдоль некоторого линейного гомеоморфизма.

Пусть $X_{1}$ и $X_{2}$ - симплициально клеточные комплексы. Отображение $X_{1} \rightarrow X_{2}$ называется симплициальным, если оно отображает каждый симплекс комплекса $X_{1}$ линейно и сюръективно на некоторый симплекс комплекса $X_{2}$. Изоморфизмом симплициально клеточных комплексов называется симплициальное отображение, имеющее симплициальное обратное.

Если во всех сформулированных определениях везде рассматривать кубы вместо симплексов, мы получим определения кубического комплекса, кубически клеточного комплекса, кубического отображения и изоморфизма кубически клеточных комплексов. Все рассматриваемые комплексы предполагаются конечными.

ЗАмечАниЕ 2.1. Термин "симплициально клеточный комплекс" использовался, например, в работах В. М. Бухштабера и Т. Е. Панова (см. [5]). Другие авторы для обозначения того же понятия использовали различные термины, в частности "псевдокомплекс" и "псевдотриангуляция". Автору эти термины кажутся менее удобными. Термин "кубически клеточный комплекс" является естественным аналогом термина "симплициально клеточный комплекс". Насколько известно автору, ранее этот термин в публикациях не встречался.

Если $X$ - симплициально клеточный комплекс, то конус cone $(X)$ и неприведенная надстройка $\Sigma X$ также являются симплициально клеточными комплексами. Барицентрическое подразделение симплициально клеточного или кубически клеточного комплекса $X$ обозначим через $X^{\prime}$. Заметим, что $X^{\prime}$ всегда является симплициальным комплексом. Через $\operatorname{Vert}(X)$ обозначим множество вершин комплекса $X$, через $C_{*}(X ; A)$ и $C^{*}(X ; A)$ - комплексы симплициальных (или кубических) цепей и коцепей симплициально клеточного (или кубически клеточного) комплекса $X$ с коэффициентами в абелевой группе $A$. Для обозначения изоморфизма симплициально клеточных или кубически клеточных комплексов мы будем использовать символ $\cong$.

Линк симплекса $\sigma$ симплициального комплекса $X$ обычно определяется как подкомплекс комплекса $X$, состоящий из всех симплексов $\tau$ таких, что $\sigma \cap \tau=\varnothing$ и существует симплекс комплекса $X$, содержащий $\sigma$ и $\tau$. В данной работе нам будет удобнее использовать другое определение, которое подходит и для симплициально клеточных, и для кубически клеточных комплексов.

ОПРЕДЕЛЕНИЕ 2.2. Пусть $X$ - симплициально клеточный или кубически клеточный комплекс, $\sigma$ - его клетка размерности $k$. Для каждой $l$-мерной клетки $\tau \supset \sigma, \tau \neq \sigma$, существует ровно $l-k$ клеток $\rho$ таких, что $\operatorname{dim} \rho=k+1$ и $\sigma \subset \rho \subset \tau$. Выпуклая оболочка барицентров всех таких клеток $\rho$ является $(l-k-1)$-мерным симплексом, который мы обозначим через $\Delta_{\sigma, \tau}$. Объединив 
симплексы $\Delta_{\sigma, \tau}$ для всех клеток $\tau \supset \sigma, \tau \neq \sigma$, мы получим симплициально клеточный комплекс, который мы будем называть линком клетки $\sigma$ в комплексе $X$ и обозначать через $\operatorname{link} \sigma$ или $\operatorname{link}_{X} \sigma$.

Несложно заметить, что в случае, когда $X$ - симплициальный комплекс, два определения линка дают канонически изоморфные симплициальные комплексы. Отметим также, что частично упорядоченное множество симплексов комплекса $\operatorname{link} \sigma$ (включающее пустой симплекс $\varnothing$ ) канонически изоморфно частично упорядоченному множеству клеток комплекса $X$, содержащих клетку $\sigma$.

Пусть $X$ - симплициально клеточный комплекс, $\sigma$ - его симплекс. Звездой симплекса $\sigma$ называется подкомплекс $\operatorname{star} \sigma \subset X$, являющийся объединением всех замкнутых симплексов комплекса $X$, содержащих симплекс $\sigma$. Если $X-$ симплициальный комплекс, то $\operatorname{star} \sigma \cong \sigma * \operatorname{link} \sigma$.

Симплициальный комплекс называется комбинаторной сферой, если он кусочно линейно гомеоморфен границе симплекса. Симплициальный комплекс называется $n$-мерным комбинаторным многообразием, если линк каждой его вершины является $(n-1)$-мерной комбинаторной сферой. Аналогично, кубический (соответственно, симплициально клеточный или кубически клеточный) комплекс называется $n$-мерным кубическим (соответственно, симплициально клеточным или кубически клеточным) комбинаторным многообразием, если линки всех его вершин кусочно линейно гомеоморфны границе $n$-мерного симплекса.

Симплициальный (соответственно, симплициально клеточный, кубический или кубически клеточный) комплекс $X$ называется $n$-мерным симплициальным (соответственно, симплициально клеточным, кубическим или кубически клеточным) псевдомногообразием, если каждая клетка комплекса $X$ содержится в некоторой $n$-мерной клетке, причем каждая $(n-1)$-мерная клетка комплекса $X$ содержится ровно в двух $n$-мерных клетках.

Все псевдомногообразия, рассматриваемые в настоящей работе, будут так называемыми нормалъными псевдомногообразиями (см. [25]). Симплициально клеточное или кубически клеточное $n$-мерное псевдомногообразие $X$ называется нормальным, если $H_{n}(X, X \backslash x) \cong \mathbb{Z}$ для всех точек $x \in X$. Эквивалентное определение: $X$ нормально, если линк каждой его клетки $\sigma$ связен при $\operatorname{dim} \sigma \leqslant n-2$. Компоненты связности нормального псевдомногообразия $X$ являются сильно связными: для любых двух $n$-мерных клеток $\tau_{1}$ и $\tau_{2}$ существует последовательность $n$-мерных клеток $\tau_{1}=\rho_{1}, \rho_{2}, \ldots, \rho_{r}=\tau_{2}$ такая, что для любого $i$ клетки $\rho_{i}$ и $\rho_{i+1}$ имеют общую $(n-1)$-мерную грань. Несложно проверить, что при $\operatorname{dim} \sigma \leqslant n-2$ линк клетки $\sigma$ нормального псевдомногообразия $X$ является связным нормальным псевдомногообразием. В частности, линк клетки $\sigma$ является сильно связным.

В дальнейшем мы будем рассматривать только нормальные псевдомногообразия и всегда будем понимать под псевдомногообразием нормальное псевдомногообразие, не оговаривая это особо. Класс нормальных псевдомногообразий включает в себя все наиболее интересные примеры псевдомногообразий: комбинаторные многообразия, гомологические многообразия, многообразия с коническими особенностями. Для произвольного $n$-мерного псевдомногообразия $X$ можно построить его нормализацию $X^{\text {norm }}[25]$. Пусть $\sigma_{1}, \sigma_{2}, \ldots, \sigma_{q}-$ 
все $n$-мерные симплексы (соответственно, кубы) псевдомногообразия $X$. Рассмотрим их несвязное объединение и произведем в нем следующие отождествления: для любых двух симплексов (кубов) $\sigma_{i}$ и $\sigma_{j}$, имеющих в $X$ общую гипергрань, отождествим их соответствующие гиперграни вдоль соответствующего изоморфизма. Несложно проверить, что полученное псевдомногообразие $X^{\text {norm }}$ является нормальным. При этом существует симплициальное (кубическое) отображение $X^{\text {norm }} \rightarrow X$, являющееся гомеоморфизмом на дополнениях к $(n-2)$-мерным остовам комплексов $X^{\text {norm }}$ и $X$.

Далее мы будем рассматривать, в основном, ориентированные псевдомногообразия. Под изоморфизмом ориентированных псевдомногообразий мы всегда понимаем изоморфизм, сохраняющий ориентацию. Изоморфизм, обращающий ориентацию, мы будем называть антиизоморфизмом. Ориентацию симплекса удобно задавать посредством упорядочения его вершин. Пусть $\sigma$ и $\tau-$ ориентированные симплексы. Тогда их джойн $\sigma * \tau$ наделяется ориентацией, задаваемой последовательностью вершин, которая получается, если к последовательности вершин, задающей ориентацию симплекса $\sigma$, приписать последовательность вершин, задающую ориентацию симплекса $\tau$. Таким же образом наделяется ориентацией джойн двух ориентированных симплициально клеточных псевдомногообразий. Пусть $\sigma$ - ориентированная клетка ориентированного псевдомногообразия $X$. Тогда линк link $\sigma$ наделяется такой ориентацией, что индуцированная ориентация комплекса $\sigma * \operatorname{link} \sigma \subset X$ совпадает с ограничением заданной ориентации псевдомногообразия $X$. Koнус $\operatorname{cone}(X)$ над ориентированным симплициально клеточным псевдомногообразием $X$ всегда наделяется такой ориентацией, что линк вершины конуса изоморфен псевдомногообразию $X$ с сохранением ориентации.

2.2. Формулировки результатов. Пусть $\left(Y_{1}, Y_{2}, \ldots, Y_{k}\right)$ - набор связных ориентированных симплициальных псевдомногообразий одинаковой размерности. Набор $\left(Y_{1}, Y_{2}, \ldots, Y_{k}\right)$ мы будем называть сбалансированным, если вершины псевдомногообразия $Y=Y_{1} \sqcup Y_{2} \sqcup \cdots \sqcup Y_{k}$ могут быть разбиты на пары так, что линки вершин в каждой паре антиизоморфны. (Иногда мы будем говорить, что сбалансированным является псевдомногообразие $Y$.) Сформулируем аналоги теорем 1.1 и 1.2 для псевдомногообразий. (Напомним, что под псевдомногообразием мы всегда понимаем нормальное псевдомногообразие.)

Теорема 2.1. Пусть $\left(Y_{1}, Y_{2}, \ldots, Y_{k}\right)$ - сбалансированный набор связных ориентированных $(n-1)$-мерных симплициальных псевдомногообразий. Тогда существует ориентированное п-мерное симпличиальное псевдомногообразие $K$, набор линков вершин которого совпадает с точностью до изоморфизма с набором

$$
(\underbrace{Y_{1}, \ldots, Y_{1}}_{r}, \underbrace{Y_{2}, \ldots, Y_{2}}_{r}, \ldots, \underbrace{Y_{k}, \ldots, Y_{k}}_{r}, Z_{1}, Z_{2}, \ldots, Z_{l},-Z_{1},-Z_{2}, \ldots,-Z_{l})
$$

для некоторого натурального числа $r$ и некоторых связных ориентированных $(n-1)$-мерных симплициальных псевдомногообразий $Z_{1}, Z_{2}, \ldots, Z_{l}$.

ТеОРема 2.2. Пусть $\left(Y_{1}, Y_{2}, \ldots, Y_{k}\right)$ - сбалансированный набор связных ориентированных $(n-1)$-мерных симплициальных псевдомногообразий. Тогда 
существует ориентированное $п$-мерное кубически клеточное псевдомногообразие $X$, набор линков вершин которого совпадает с точностью до изоморфизма с набором

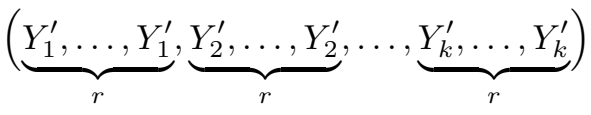

для некоторого натуралъного числа $r$.

Наша главная задача в этом параграфе - дать явные конструкции таких псевдомногообразий $K$ и $X$. Теорема 1.2 является непосредственным следствием теоремы 2.2. Действительно, если $Y_{1}, Y_{2}, \ldots, Y_{k}$ - комбинаторные сферы, псевдомногообразие $X$ автоматически будет кубически клеточным комбинаторным многообразием. Явная конструкция псевдомногообразия $X$ будет дана в пп. 2.3-2.5.

Теорема 1.1 не следует непосредственно из теоремы 2.1: даже если $Y_{1}$, $Y_{2}, \ldots, Y_{k}$ - комбинаторные сферы, псевдомногообразие $K$ может не быть комбинаторным многообразием в силу того, что какое-нибудь из псевдомногообразий $Z_{1}, Z_{2}, \ldots, Z_{l}$ не будет комбинаторной сферой. Тем не менее, имеется явная конструкция, которая дает искомый симплициальный комплекс $K$ и в условиях теоремы 1.1, и в условиях теоремы 2.1. Отметим, что основная сложность этой конструкции заключена в построении комплекса $X$; комплекс $K$ строится по комплексу $X$ довольно легко. Чтобы не загромождать изложение, мы в пп. 2.7, 2.8 рассматриваем случай комбинаторных сфер $Y_{1}, Y_{2}, \ldots, Y_{k}$. Случай псевдомногообразий полностью аналогичен. Теоремы, аналогичные теоремам 1.1 и 2.1, имеют место и для некоторых промежуточных классов между классом комбинаторных многообразий и классом всех симплициальных псевдомногообразий, например, для симплициальных гомологических многообразий (см. п. 3.3).

Пусть $X$ - симплициально клеточное или кубически клеточное псевдомногообразие. Введем обозначение

$$
\mathcal{L}(X)=\bigsqcup_{x \in \operatorname{Vert}(X)} \operatorname{link} x .
$$

Вершины псевдомногообразия $\mathcal{L}(X)$ находятся во взаимно однозначном соответствии с ориентированными ребрами псевдомногообразия $X$. Операция изменения ориентации ребра задает инволюцию $\lambda_{X}: z \mapsto \tilde{z}$ на множестве вершин псевдомногообразия $\mathcal{L}(X)$ и набор антиизоморфизмов

$$
\chi_{X, z}: \operatorname{star} z \rightarrow \operatorname{star} \tilde{z}, \quad z \in \operatorname{Vert}(\mathcal{L}(X)),
$$

такой, что $\chi_{X, \tilde{z}}=\chi_{X, z}^{-1}$.

С другой стороны, пусть $\left(Y_{1}, Y_{2}, \ldots, Y_{k}\right)$ - сбалансированный набор $(n-1)$ мерных симплициальных псевдомногообразий. Вершины псевдомногообразия $Y=Y_{1} \sqcup Y_{2} \sqcup \cdots \sqcup Y_{k}$ могут быть разбиты на пары с антиизоморфными линками, или, что то же самое, с антиизоморфными звездами. Значит, существуют инволюция $\lambda: y \mapsto \tilde{y}$ на множестве $\operatorname{Vert}(Y)$ и набор антиизоморфизмов

$$
\chi_{y}: \operatorname{star} y \rightarrow \operatorname{star} \tilde{y}, \quad y \in \operatorname{Vert}(Y),
$$


такой, что $\chi_{\tilde{y}}=\chi_{y}^{-1}$. Очевидно, что инволюция $\lambda$ и антиизоморфизмы $\chi_{y}$ могут быть определены неоднозначно. Фиксируем какой-нибудь выбор инволюции $\lambda$ и антиизоморфизмов $\chi_{y}$. Рассмотрим множество вершин псевдомногообразия $Y^{\prime}$. Его можно представить в виде

$$
\operatorname{Vert}\left(Y^{\prime}\right)=\bigsqcup_{j=1}^{n} W_{j}(Y),
$$

где $W_{j}(Y)$ - множество барицентров $(j-1)$-мерных симплексов комплекса $Y$ (в частности, $\left.W_{1}(Y)=\operatorname{Vert}(Y)\right)$.

Конструкция, описанная в пп. 2.3-2.5, даст нам более сильное утверждение, чем теорема 2.2: в построенном псевдомногообразии $X$ инволюция $\lambda_{X}$ и набор антиизоморфизмов $\chi_{X, z}$ будут согласованы с инволюцией $\lambda$ и набором антиизоморфизмов $\chi_{y}$. Более точно, мы получим следующее утверждение.

ТеОрема 2.3. Пусть $Y$ - ориентированное $(n-1)$-мерное симплициальное псевдомногообразие, $\lambda: y \mapsto \tilde{y}-$ инволюиия на множестве $\operatorname{Vert}(Y), \chi_{y}$ : $\operatorname{star} y \rightarrow \operatorname{star} \tilde{y}$ - антиизоморфизмы такие, что $\chi_{\tilde{y}}=\chi_{y}^{-1}$. Тогда существуют ориентированное $n$-мерное кубически клеточное псевдомногообразие $X u$ изоморбизм $\mathcal{L}(X) \cong Y^{\prime} \times S$, где $S$ - конечное множество, такие, что:

1) подмножества $W_{j}(Y) \times S \subset \operatorname{Vert}\left(Y^{\prime}\right) \times S=\operatorname{Vert}(\mathcal{L}(X))$ инвариантны относительно инволюиии $\lambda_{X}$;

2) если $y \in \operatorname{Vert}(Y), s \in S$, mо $\lambda_{X}(y, s)=\left(\lambda(y), s_{1}\right)$ для некоторого элемента $s_{1} \in S$ (зависящего от у и $\left.s\right)$;

3) если $y \in \operatorname{Vert}(Y), s \in S$, то антиизоморфизм

$$
\chi_{X,(y, s)}: \operatorname{star}_{Y^{\prime} \times S}(y, s) \rightarrow \operatorname{star}_{Y^{\prime} \times S}\left(\lambda(y), s_{1}\right)
$$

индущирован антиизоморфизмом $\chi_{y}: \operatorname{star}_{Y} y \rightarrow \operatorname{star}_{Y} \lambda(y)$.

Заметим, что теорема 2.3 является содержательной, даже если все комбинаторные сферы $Y_{i}$ изоморфны границе $n$-мерного симплекса. Именно в этом случае она будет использоваться в п. 4.6.

2.3. Псевдомногообразия, построенные по графам. В настоящей статье граф - это всегда неориентированный конечный граф, который может содержать кратные ребра, но не содержит петель. Граф называется однородным, если степени всех его вершин одинаковы. Пусть Г - однородный граф степени $n$. Будем говорить, что ребра графа Г раскрашены правильным образом в цвета из некоторого $n$-элементного множества $A$, если каждому ребру графа $\Gamma$ поставлен в соответствие цвет - элемент множества $A$ - так, что из каждой вершины выходит $n$ ребер попарно различных цветов.

Пусть $V$ - множество вершин графа Г. Каждому цвету $a \in A$ поставим в соответствие отображение $\Phi_{a}: V \rightarrow V$, переводящее вершину $v \in V$ в вершину, соединенную с $v$ ребром цвета $a$. Тогда $\Phi_{a}-$ инволюция без неподвижных точек. Обратно, если нам заданы произвольное конечное множество $V$ и набор инволюций без неподвижных точек $\Phi_{a}: V \rightarrow V, a \in A$, то мы можем построить однородный граф $Г$ на множестве вершин $V$ с ребрами, раскрашенными правильным образом в цвета из множества $A$. Для этого нужно для любой 
вершины $v \in V$ и любого элемента $a \in A$ соединить вершины $v$ и $\Phi_{a}(v)$ ребром цвета $a$.

Пусть $Y-(n-1)$-мерное симплициально клеточное псевдомногообразие. Раскраска вершин псевдомногообразия $Y$ в цвета из $n$-элементного множества $A$ называется правильной, если вершины каждого симплекса раскрашены в попарно различные цвета. Есть один важный случай, когда вершины псевдомногообразия $Y$ могут быть естественным образом раскрашены в $n$ цветов. Это случай, когда псевдомногообразие $Y$ является барицентрическим подразделением некоторого симплициально клеточного псевдомногообразия $Z$. Раскраска получается следующим образом: вершину, являющуюся барицентром $j$-мерного симплекса комплекса $Z$, красим в цвет $j+1$.

Оказывается, что $(n-1)$-мерные нормальные симплициально клеточные псевдомногообразия с правильной раскраской вершин находятся во взаимно однозначном соответствии с однородными графами степени $n$, имеющими правильную раскраску ребер. Конструкция, устанавливающая это соответствие, принадлежит М. Пеццана [26] в размерности три и М. Ферри [27] в общем случае (см. также [28]). (М. Пеццана и М. Ферри не рассматривали условие нормальности, поэтому у них соответствие не было взаимно однозначным.) Сформулируем в удобном для нас виде эту конструкцию. Без ограничения общности можно считать, что $A=\{1,2, \ldots, n\}$.

Построение графа по псевдомногообразию. Пусть $Y-(n-1)$-мерное симплициально клеточное псевдомногообразие с правильной раскраской вершин. Каждому $(n-1)$-мерному симплексу $\sigma$ комплекса $Y$ поставим в соответствие вершину $v_{\sigma}$. Каждому $(n-2)$-мерному симплексу $\tau$ комплекса $Y$ поставим в соответствие ребро $e_{\tau}$, соединяющее вершины $v_{\sigma_{1}}$ и $v_{\sigma_{2}}$, где $\sigma_{1}$ и $\sigma_{2}$ - два $(n-1)$-мерных симплекса, содержащих $\tau$. Покрасим ребро $e_{\tau}$ в цвет $j$, если симплекс $\tau$ не содержит вершины цвета $j$.

Построение псевдомногообразия по графу. Пусть $V$ - множество вершин графа Г и $\Phi_{1}, \Phi_{2}, \ldots, \Phi_{n}$ - соответствующие инволюции. Пусть

$$
\Delta^{n-1}=\left\{\left(t_{1}, t_{2}, \ldots, t_{n}\right) \in \mathbb{R}^{n} \mid t_{1}+t_{2}+\cdots+t_{n}=1, t_{j} \geqslant 0, j=1,2, \ldots, n\right\}
$$

- стандартный $(n-1)$-мерный симплекс. Положим,

$$
\mathbf{K}(\Gamma)=V \times \Delta^{n-1} / \sim,
$$

где отношение эквивалентности $\sim$ порождено отождествлениями

$$
\left(v, t_{1}, t_{2}, \ldots, t_{n}\right) \sim\left(\Phi_{j}(v), t_{1}, t_{2}, \ldots, t_{n}\right), \quad \text { если } \quad t_{j}=0 .
$$

Непосредственно проверяется, что $\mathbf{K}(\Gamma)$ - нормальное псевдомногообразие. Раскраска вершин комплекса $\mathbf{K}(\Gamma)$ индуцируется стандартной раскраской вершин симплекса $\Delta^{n-1}$. Несложно проверить, что приведенные конструкции являются взаимно обратными.

Частично упорядоченное множество симплексов комплекса $\mathbf{K}(\Gamma)$. Для любого подмножества $B \subset A$ обозначим через $\Gamma_{B}$ граф, получаемый из $\Gamma$ при удалении всех ребер, цвета которых не принадлежат $B$. Тогда $\Gamma_{B}-$ однородный граф степени, равной количеству элементов в множестве $B$. Обозначим через $\mathcal{K}(\Gamma)$ множество, элементами которого являются всевозможные пары $(B, \Upsilon)$, 
где $B$ - подмножество множества $A, B \neq A$, и $\Upsilon$ - компонента связности графа $\Gamma_{B}$. Введем упорядочение в множестве $\mathcal{K}(\Gamma)$, положив $\left(B_{1}, \Upsilon_{1}\right) \leqslant\left(B_{2}, \Upsilon_{2}\right)$ тогда и только тогда, когда $B_{2} \subset B_{1}$ и $\Upsilon_{2} \subset \Upsilon_{1}$.

ПРЕДЛОЖЕНИЕ 2.1. Частично упорядоченное по включению множество симплексов комплекса $\mathbf{K}(\Gamma)$ (не включающее пустой симплекс) изоморфно частично упорядоченному множеству $\mathcal{K}(\Gamma)$. При этом изоморфизме каждому симплексу $\sigma$ комплекса $\mathbf{K}(\Gamma)$ соответствует пара $(B, \Upsilon)$, где $B \subset A$ подмножество, состоящее из всех иветов, не являющихся иветами вершин симплекса $\sigma$.

ДокАЗАТЕЛьство. Через $\Delta_{v}$ мы будем обозначать $(n-1)$-мерный симплекс комплекса $\mathbf{K}(\Gamma)$, соответствующий вершине $v \in V$. Для каждого подмножества $B \varsubsetneqq A$ обозначим через $\Delta_{B, v}$ грань симплекса $\Delta_{v}$, натянутую на множество вершин, цвета которых не принадлежат подмножеству $B$. Тогда $\Delta_{B, v}-$ симплекс размерности $n-k-1$, где $k$ - количество элементов в множестве $B$. Очевидно, что каждый симплекс комплекса $\mathbf{K}(\Gamma)$ имеет вид $\Delta_{B, v}$ для некоторых $B$ и $v$. Рассматривая отождествления, производимые при склейке комплекса $\mathbf{K}(\Gamma)$, мы получаем, что $\Delta_{B, v_{1}}=\Delta_{B, v_{2}}$, если вершины $v_{1}$ и $v_{2}$ соединены ребром, цвет которого принадлежит множеству $B$. Значит, $\Delta_{B, v_{1}}=\Delta_{B, v_{2}}$, если вершины $v_{1}$ и $v_{2}$ лежат в одной компоненте связности графа $\Gamma_{B}$. Несложно также проверить, что симплексы $\Delta_{B, v_{1}}$ и $\Delta_{B, v_{2}}$ различны, если вершины $v_{1}$ и $v_{2}$ лежат в разных компонентах связности графа $\Gamma_{B}$.

Симплекс комплекса $\mathbf{K}(\Gamma)$, соответствующий паре $(B, \Upsilon)$, мы будем обозначать через $\Delta_{B, \Upsilon}$. Для $(n-1)$-мерных симплексов мы, как правило, вместо обозначения $\Delta_{\varnothing, v}$ будем использовать обозначение $\Delta_{v}$.

ПРЕДЛОЖЕНИЕ 2.2. Линк симплекса $\Delta_{B, \Upsilon}$ в симплициально клеточном комплексе $\mathbf{K}(\Gamma)$ изоморфен симплициально клеточному комплексу $\mathbf{K}(\Upsilon)$.

ДоКАЗАТЕЛЬСтво. Несложно проверить, что частично упорядоченное множество $\mathcal{K}(\Upsilon)$ изоморфно интервалу $((B, \Upsilon),+\infty)$ в частично упорядоченном множестве $\mathcal{K}(\Gamma)$. Значит, оно изоморфно частично упорядоченному множеству симплексов комплекса $\mathbf{K}(\Gamma)$, содержащих симплекс $\Delta_{B, \Upsilon}$ и не совпадающих с ним. Таким образом, частично упорядоченное множество симплексов комплекса $\mathbf{K}(\Upsilon)$ изоморфно частично упорядоченному множеству симплексов комплекса $\operatorname{link} \Delta_{B, \Upsilon \text {. }}$

Ориентация. Несложно проверить, что псевдомногообразие $\mathbf{K}(\Gamma)$ является ориентируемым тогда и только тогда, когда граф $Г$ не содержит циклов нечетной длины, т. е. является двудольным. Предположим, что задано разбиение множества $V$ на два непересекающихся подмножества $V_{+}$и $V_{-}$такое, что граф Г является двудольным по отношению к этому разбиению. Если $v \in V_{+}$, зададим на симплексе $\Delta_{v}$ ориентацию, индуцированную канонической ориентацией стандартного симплекса $\Delta^{n-1} \subset \mathbb{R}^{n}$. Если $v \in V_{-}$, зададим на симплексе $\Delta_{v}$ ориентацию, противоположную ориентации, индуцированной канонической ориентацией стандартного симплекса $\Delta^{n-1} \subset \mathbb{R}^{n}$. Легко проверить, что введенные ориентации на симплексах $\Delta_{v}$ согласованы. Таким образом, разбиение 
$V=V_{+} \sqcup V_{-}$задает ориентацию псевдомногообразия $\mathbf{K}(\Gamma)$. Обратно, ориентация псевдомногообразия $\mathbf{K}(\Gamma)$ задает разбиение множества $V$, относительно которого граф Г является двудольным.

В дальнейшем нам понадобится следующий аналог конструкции ПеццанаФерри для кубически клеточных комплексов. Пусть Г - однородный граф степени $2 n$, ребра которого раскрашены правильным образом в цвета из множества

$$
A=\left\{1^{0}, 2^{0}, \ldots, n^{0}, 1^{1}, 2^{1}, \ldots, n^{1}\right\} .
$$

Пусть $V$ - множество вершин графа Г. Инволюцию, соответствующую цвету $j^{e}$, обозначим через $\Phi_{j}^{e}$. Графу $Г$ может быть поставлено в соответствие нормальное $n$-мерное кубически клеточное псевдомногообразие

$$
\mathbf{Q}(\Gamma)=V \times I^{n} / \sim
$$

где $I=[0,1]$ и отношение эквивалентности $\sim$ порождено отождествлениями

$$
\begin{aligned}
& \left(v, t_{1}, t_{2}, \ldots, t_{n}\right) \sim\left(\Phi_{j}^{0}(v), t_{1}, t_{2}, \ldots, t_{n}\right), \quad \text { если } \quad t_{j}=0, \\
& \left(v, t_{1}, t_{2}, \ldots, t_{n}\right) \sim\left(\Phi_{j}^{1}(v), t_{1}, t_{2}, \ldots, t_{n}\right), \quad \text { если } \quad t_{j}=1 .
\end{aligned}
$$

Tипом вершины $x$ куба $v \times I^{n}$ будем называть набор координат соответствующей вершины стандартного куба $I^{n}$. Заметим, что отношение эквивалентности $\sim$ отождествляет вершины кубов, только если они имеют одинаковый тип. Поэтому для каждой вершины комплекса $\mathbf{Q}(\Gamma)$ корректно определен ее тип - $n$-мерный вектор, каждая координата которого равна 0 или 1 . Пусть $\mathbf{e}=\left(e_{1}, e_{2}, \ldots, e_{n}\right)-$ вектор, каждая координата которого равна 0 или 1 . Введем обозначения

$$
A_{\mathbf{e}}=\left\{1^{e_{1}}, 2^{e_{2}}, \ldots, n^{e_{n}}\right\}, \quad \Gamma_{\mathbf{e}}=\Gamma_{A_{\mathbf{e}}} .
$$

Подмножество $B \subset A$ мы будем называть правильным, если для любого $j$ подмножеству $B$ принадлежит не более чем один из элементов $j^{0}$ и $j^{1}$. Обозначим через $\mathcal{Q}(\Gamma)$ множество, элементами которого являются всевозможные пары $(B, \Upsilon)$, где $B$ - правильное подмножество множества $A$ и $\Upsilon$ - компонента связности графа $\Gamma_{B}$. Упорядочение в множестве $\mathcal{Q}(\Gamma)$ вводится точно так же, как в $\mathcal{K}(\Gamma)$. Следующие два предложения аналогичны предложениям 2.1 и 2.2 .

ПРЕДЛОЖЕНИЕ 2.3. Частично упорядоченное по включению множество кубов комплекса $\mathbf{Q}(\Gamma)$ (не включающее пустой куб) изоморфно частично упорядоченному множеству $\mathcal{Q}(\Gamma)$. При этом изоморфизме каждому кубу $\sigma$ комплекса $\mathbf{Q}(\Gamma)$ соответствует пара $(B, \Upsilon)$, где $B \subset A$ - подмножество, являющееся пересечением всех подмножеств $A_{\mathbf{e}}$ таких, что куб $\sigma$ содержит вершину типа е. В частности, вершинам типа е соответствуют пары $\left(A_{\mathbf{e}}, \Upsilon\right)$, где $\Upsilon$ - компоненты связности графа $\Gamma_{\mathbf{e}}$.

Куб комплекса $\mathbf{Q}(\Gamma)$, соответствующий паре $(B, \Upsilon)$, мы будем обозначать через $\square_{B, \Upsilon}$. Для $n$-мерных кубов мы, как правило, вместо обозначения $\square_{\varnothing, v}$ будем использовать обозначение $\square_{v}$.

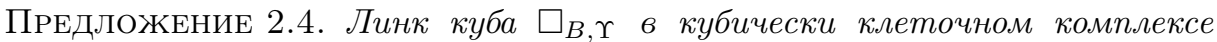
$\mathbf{Q}(\Gamma)$ изоморфен симпличиально клеточному комплексу $\mathbf{K}(\Upsilon)$. 
Псевдомногообразие $\mathbf{Q}(\Gamma)$ является ориентируемым тогда и только тогда, когда граф Г не содержит циклов нечетной длины. Разбиение множества $V$ на два непересекающихся подмножества $V_{+}$и $V_{-}$, относительно которого граф $\Gamma$ является двудольным, задает ориентацию псевдомногообразия $\mathbf{Q}(\Gamma)$.

Пусть $x=\square_{A_{\mathbf{e}}, \Upsilon}-$ произвольная вершина типа е. Из предложения 2.4 следует, что линк вершины $x$ изоморфен комплексу $\mathbf{K}(\Upsilon)$. Предположим теперь, что граф Г двудольный. Тогда ориентация псевдомногообразия $\mathbf{Q}(\Gamma)$ индуцирует ориентацию комплекса link $x$. C другой стороны, забывая о верхних индексах у цветов, мы можем считать, что ребра графа $\Gamma_{\mathbf{e}}$ раскрашены правильным образом в цвета $1,2, \ldots, n$. Разбиение графа $\Gamma$ на две доли задает разбиение графа $\Gamma_{\mathbf{e}}$ на две доли. Следовательно, граф $\Gamma_{\mathbf{e}}$ и его связная компонента $\Upsilon$ - двудольные графы с ребрами, раскрашенными правильным образом в цвета $1,2, \ldots, n$. Таким образом, симплициально клеточное псевдомногообразие $\mathbf{K}(\Upsilon)$ получает выделенную ориентацию. Сохраняет ли ориентацию изоморфизм комплексов $\operatorname{link} x$ и $\mathbf{K}(\Upsilon)$ ? Непосредственно проверяется, что имеет место следующее утверждение.

ПРЕДЛОЖЕНИЕ 2.5. Изоморфизм из предложения 2.4 между комплексами $\operatorname{link} x$ и $\mathbf{K}(\Upsilon)$ сохраняет ориентацию тогда и только тогда, когда число $e_{1}+$ $e_{2}+\cdots+e_{n}$ четно.

2.4. Переход к большим кубам. Рассмотрим куб $[0,1]^{n} \subset \mathbb{R}^{n}$. Разобьем его на $2^{n}$ кубов гиперплоскостями $t_{j}=\frac{1}{2}$. Полученное разбиение мы будем называть каноническим подразделением стандартного куба. Пусть теперь $X-$ произвольный кубически клеточный комплекс. Подразделим каждый его куб каноническим образом. Полученный кубически клеточный комплекс мы будем называть каноническим подразделением комплекса $X$.

В этом пункте мы покажем, что если граф Г удовлетворяет некоторым специальным условиям, то комплекс $\mathbf{Q}(\Gamma)$ является каноническим подразделением некоторого кубически клеточного комплекса $\widetilde{\mathbf{Q}}(\Gamma)$.

ПРЕДЛОжЕНИЕ 2.6. Предположим, что граф Г удовлетворяет следующим условиям:

1) $\Phi_{i}^{1} \circ \Phi_{j}^{1}=\Phi_{j}^{1} \circ \Phi_{i}^{1}$ для любъх $i u j$;

2) $\Phi_{i}^{0} \circ \Phi_{j}^{1}=\Phi_{j}^{1} \circ \Phi_{i}^{0}$, если $i \neq j$;

3) существует отображение $h: V \rightarrow \mathbb{Z}_{2}^{n}$ такое, что

$$
\begin{aligned}
h\left(\Phi_{i}^{0}(v)\right) & =h(v), & i & =1,2, \ldots, n, \\
h\left(\Phi_{i}^{1}(v)\right) & =h(v)+\varepsilon_{i}, & i & =1,2, \ldots, n,
\end{aligned}
$$

где $\left(\varepsilon_{1}, \ldots, \varepsilon_{n}\right)-$ базис группъ $\mathbb{Z}_{2}^{n}$.

Тогда псевдомногообразие $\mathbf{Q}(\Gamma)$ является каноническим подразделением некоторого кубически клеточного псевдомногообразия $\widetilde{\mathbf{Q}}(\Gamma)$.

ДокАзАтЕльство. Из свойства 1) следует, что инволюции $\Phi_{j}^{1}: V \rightarrow V$ задают действие группы $\mathbb{Z}_{2}^{n}$ на множестве $V$. Кроме того, из свойства 3) вытекает, что это действие свободное. Введем обозначение $\widetilde{V}=V / \mathbb{Z}_{2}^{n}$. Пусть $p: V \rightarrow \widetilde{V}-$ отображение факторизации. Из свойства 3) легко выводится, что отображение $p \times h: V \rightarrow \widetilde{V} \times \mathbb{Z}_{2}^{n}$ является биекцией. Поэтому мы можем рассматривать 
инволюции $\Phi_{j}^{e}$ как инволюции на множестве $\widetilde{V} \times \mathbb{Z}_{2}^{n}$. Определим отображения $\widetilde{\Phi}_{j}^{e}: \widetilde{V} \rightarrow \widetilde{V}$ по формулам

$$
\widetilde{\Phi}_{j}^{e}(u)=p\left(\Phi_{j}^{0}\left(u, e \varepsilon_{j}\right)\right) .
$$

Тогда $\Phi_{j}^{0}\left(u, e \varepsilon_{j}\right)=\left(\widetilde{\Phi}_{j}^{e}(u), e \varepsilon_{j}\right)$. Следовательно, $\widetilde{\Phi}_{j}^{e}\left(\widetilde{\Phi}_{j}^{e}(u)\right)=u$. Кроме того, из равенства $\widetilde{\Phi}_{j}^{e}(u)=u$ следовало бы равенство $\Phi_{j}^{0}\left(u, e \varepsilon_{j}\right)=\left(u, e \varepsilon_{j}\right)$, что невозможно. Таким образом, отображения $\widetilde{\Phi}_{j}^{e}-$ инволюции без неподвижных точек. Эти инволюции задают однородный граф степени $2 n$ на множестве $\widetilde{V}$ с ребрами, раскрашенными правильным образом в цвета из множества $A$. Обозначим этот граф через $\widetilde{\Gamma}$. Положим $\widetilde{\mathbf{Q}}(\Gamma)=\mathbf{Q}(\widetilde{\Gamma})$. Несложно проверить, что каноническое подразделение комплекса $\widetilde{\mathbf{Q}}(\Gamma)$ изоморфно комплексу $\mathbf{Q}(\Gamma)$.

Отметим, что кубически клеточное псевдомногообразие $\widetilde{\mathbf{Q}}(\Gamma)$ может быть задано следующим образом:

$$
\widetilde{\mathbf{Q}}(\Gamma)=V \times I^{n} / \sim,
$$

где отношение эквивалентности $\sim$ порождено отождествлениями

$$
\begin{aligned}
\left(v, t_{1}, t_{2}, \ldots, t_{n}\right) & \sim\left(\Phi_{j}^{0}(v), t_{1}, t_{2}, \ldots, t_{n}\right), \quad \text { если } \quad t_{j}=0, \\
\left(v, t_{1}, \ldots, t_{j-1}, t_{j}, t_{j+1}, \ldots, t_{n}\right) & \sim\left(\Phi_{j}^{1}(v), t_{1}, \ldots, t_{j-1}, 1-t_{j}, t_{j+1}, \ldots, t_{n}\right) .
\end{aligned}
$$

Вершины комплекса $\mathbf{Q}(\Gamma)$ - это в точности барицентры граней комплекса $\widetilde{\mathbf{Q}}(\Gamma)$. При этом вершины комплекса $\mathbf{Q}(\Gamma)$, имеющие тип $\mathbf{0}=(0, \ldots, 0),-$ это в точности вершины комплекса $\widetilde{\mathbf{Q}}(\Gamma)$. Очевидно, что для любой вершины комплекса $\widetilde{\mathbf{Q}}(\Gamma)$ ее линки в комплексах $\widetilde{\mathbf{Q}}(\Gamma)$ и $\mathbf{Q}(\Gamma)$ изоморфны.

2.5. Конструкция псевдомногообразия $X$. В этом пункте мы дадим явную конструкцию кубически клеточного псевдомногообразия $X$ из теоремы 2.3.

Покрасим барицентр каждого $j$-мерного симплекса комплекса $Y$ в цвет $j+1$. Таким образом, $Y^{\prime}$ - симплициальное псевдомногообразие с вершинами, раскрашенными правильным образом в $n$ цветов. Обозначим через $\Upsilon$ однородный граф степени $n$, соответствующий этому псевдомногообразию. Наша цель - построить такой однородный граф Г степени $2 n$ с правильной раскраской ребер в цвета $1^{0}, 2^{0}, \ldots, n^{0}, 1^{1}, 2^{1}, \ldots, n^{1}$, что $\widetilde{\mathbf{Q}}(\Gamma)$ - искомое псевдомногообразие $X$.

Пусть $U-$ множество вершин графа $\Upsilon$. Ориентация псевдомногообразия $Y$ задает разбиение $U=U_{+} \sqcup U_{-}$, относительно которого граф $\Upsilon$ является двудольным. Обозначим через $\Phi_{1}, \ldots, \Phi_{n}$ инволюции, задающие граф $\Upsilon$. Пусть $S$ - произвольное конечное множество из $r$ элементов. Введем обозначения

$$
V=U \times S, \quad V_{+}=U_{+} \times S, \quad V_{-}=U_{-} \times S .
$$

Определим инволюции $\Phi_{j}^{0}: V \rightarrow V$ по формулам $\Phi_{j}^{0}(u, s)=\left(\Phi_{j}(u), s\right)$.

ПреДЛОЖЕНИЕ 2.7. Предположим, ито $\Phi_{j}^{1}: V \rightarrow V, j=1,2, \ldots, n,-$ инволючии такие, что $\Phi_{j}^{1}\left(V_{+}\right)=V_{-}, \Phi_{j}^{1}\left(V_{-}\right)=V_{+}$, и инволющии $\Phi_{j}^{0}, \Phi_{j}^{1}$ удовлетворяют условиям 1)-3) из предложения 2.6. Пусть Г- граф, заданный 
инволюииями $\Phi_{j}^{0}, \Phi_{j}^{1}$. Тогда $X=\widetilde{\mathbf{Q}}(\Gamma)$ - ориентированное кубически клеточное псевдомногообразие с rk вериинами, которые могут быть разбиты на $k$ групп по $r$ вершин в каждой так, что линк каждой вершины в l-й группе изоморфен псевдомногообразию $Y_{l}^{\prime}$.

ДокАЗАТЕЛЬСтво. Из предложения 2.6 следует, что псевдомногообразие $\widetilde{\mathbf{Q}}(\Gamma)$ корректно определено. Граф Г двудольный по отношению к разбиению $V=V_{+} \sqcup V_{-}$. Поэтому псевдомногообразие $\widetilde{\mathbf{Q}}(\Gamma)$ ориентированно.

Обозначим через $\Upsilon_{l}$ граф, соответствующий псевдомногообразию $Y_{l}^{\prime}$. Графы $\Upsilon_{l}$ связны, и

$$
\Upsilon=\Upsilon_{1} \sqcup \Upsilon_{2} \sqcup \cdots \sqcup \Upsilon_{k}
$$

Вершины псевдомногообразия $\widetilde{\mathbf{Q}}(\Gamma)$ - это вершины псевдомногообразия $\mathbf{Q}(\Gamma)$, имеющие тип $\mathbf{0}=(0, \ldots, 0)$. Следовательно, они находятся во взаимно однозначном соответствии с компонентами связности графа $\Gamma_{\mathbf{0}}$. Граф $\Gamma_{\mathbf{0}}$ получается из графа Г после удаления всех ребер цветов $1^{1}, 2^{1}, \ldots, n^{1}$. Поэтомy

$$
\Gamma_{\mathbf{0}} \cong \Upsilon^{\sqcup r}=\left(\Upsilon_{1} \sqcup \Upsilon_{2} \sqcup \cdots \sqcup \Upsilon_{k}\right)^{\sqcup r}
$$

Из предложений 2.4 и 2.5 следует, что псевдомногообразие $\widetilde{\mathbf{Q}}(\Gamma)$ имеет $r k$ вершин, которые могут быть разбиты на $k$ групп по $r$ вершин в каждой так, что линк каждой вершины в $l$-й группе изоморфен комплексу $\mathbf{K}\left(\Upsilon_{l}\right) \cong Y_{l}^{\prime}$.

Таким образом, наша цель - найти конечное множество $S$ и инволюции $\Phi_{j}^{1}$, удовлетворяющие условиям предложения 2.7. Сначала мы проведем построение в следующем частном случае.

ПРЕДПОЛОЖЕНИЕ. Каждой вершине симплициалъного псевдомногообразия $Y$ сопоставлена метка из некоторого конечного множества $\mathcal{C}$ так, что выполнены следующие условия:

1) для любой вершины $y \in Y$ вершины подкомплекса star $y \subset Y$ имеют попарно различные метки;

2) для любой вершины $y \in Y$ антиизоморфизм $\chi_{y}:$ star $y \rightarrow \operatorname{star} \tilde{y}$ сохраняет метки вершин.

Обозначим через $W$ множество всех непустых симплексов комплекса $Y$. Вершины графа $\Upsilon$ находятся во взаимно однозначном соответствии с последовательностями

$$
\sigma^{0} \subset \sigma^{1} \subset \cdots \subset \sigma^{n-1}, \quad \sigma^{j} \in W .
$$

Вершину, соответствующую такой последовательности, мы будем обозначать через $u\left(\sigma^{0}, \sigma^{1}, \ldots, \sigma^{n-1}\right)$. Изоморфизмом (антиизоморфизмом) симплициальных комплексов с помеченными вершинами мы будем в дальнейшем называть изоморфизм (антиизоморфизм), сохраняющий метки вершин. Для любого симплекса $\sigma \in W$ все вершины звезды симплекса $\sigma$ имеют разные метки. Поэтому звезда симплекса $\sigma$ не имеет антиавтоморфизмов и для произвольных симплексов $\sigma_{1}, \sigma_{2} \in W$ существует не более одного антиизоморфизма star $\sigma_{1} \rightarrow \operatorname{star} \sigma_{2}$.

Для каждого $j=1,2, \ldots, n$ построим конечный граф $G_{j}$. Множество вершин графа $G_{j}$ совпадает с множеством $(j-1)$-мерных симплексов комплекса $Y$. Из каждой вершины $\sigma$ графа $G_{j}$ выходит ровно $j$ ребер: для каждой вершины $y$ симплекса $\sigma$ вершина $\sigma$ соединяется ребром с вершиной $\chi_{y}(\sigma)$. (Граф $G_{j}$ может содержать кратные ребра.) 
ПреДлОЖЕНИЕ 2.8. Все ицклъ в графе $G_{j}$ имеют четную длину.

ДокАзАтельство. Если два $(j-1)$-мерных симплекса комплекса $Y$ соединены ребром в графе $G_{j}$, то их звезды антиизоморфны. Поэтому из существования цикла нечетной длины следовало бы наличие антиавтоморфизма у звезды одного из симплексов комплекса $Y$, что невозможно.

СледСтвиЕ 2.1. Каждая компонента связности графа $G_{j}$ является двудольным графом с равным количеством вершин в обеих долях.

Обозначим через $G$ несвязное объединение графов $G_{1}, G_{2}, \ldots, G_{n}$. Множество вершин графа $G$ совпадает с множеством $W$. Обозначим через $P$ множество всех инволюций $\Lambda: W \rightarrow W$ таких, что для любого $\sigma \in W$ вершины $\sigma$ и $\Lambda(\sigma)$ графа $G$ лежат в одной его компоненте связности, но в разных ее долях. Из следствия 2.1 вытекает, что множество $P$ непусто. Из определения графа $G$ непосредственно следует, что звезды симплексов $\sigma$ и $\Lambda(\sigma)$ антиизоморфны для любых $\Lambda \in P, \sigma \in W$. Заметим также, что $\Lambda(y)=\tilde{y}$ для любой инволюции $\Lambda \in P$ и любой вершины $y$ комплекса $Y$. Положим

$$
S=P \times \mathbb{Z}_{2}^{n}, \quad V=U \times S, \quad V_{ \pm}=U_{ \pm} \times S .
$$

Для того чтобы определить инволюции $\Phi_{j}^{1}$, нам будут нужны некоторые дополнительные конструкции.

Для каждого симплекса $\sigma \in W$ обозначим через $c(\sigma)$ множество всех меток его вершин. Множество $c(\sigma)$ мы будем называть меткой симплекса $\sigma$. Так как все вершины симплекса $\sigma$ имеют различные метки, то множество $c(\sigma)$ содержит ровно $\operatorname{dim} \sigma+1$ элементов. Отображение $c$ можно интерпретировать как симплициальное отображение $Y \rightarrow \Delta^{|\mathcal{C}|-1}$. Пусть $c \subset \mathcal{C}-$ произвольное подмножество. Обозначим через $W_{c} \subset W$ множество, состоящее из всех симплексов $\sigma$ таких, что в комплексе $\operatorname{star} \sigma$ есть (единственный) симплекс $\rho$, для которого $c(\rho)=c$. Пусть $\Lambda \in P$. Определим инволюцию $\Lambda_{c}: W_{c} \rightarrow W_{c}$ следующим образом. Для каждого симплекса $\sigma \in W_{c}$ в качестве $\Lambda_{c}(\sigma)$ возьмем образ симплекса $\sigma$ при (единственном) антиизоморфизме $\operatorname{star} \rho \rightarrow \operatorname{star} \Lambda(\rho)$, где $\rho-$ симплекс в $\operatorname{star} \sigma$ такой, что $c(\rho)=c$.

Для каждого подмножества $c \subset \mathcal{C}$ определим отображение $\Theta_{c}: P \rightarrow P$ по формуле

$$
\Theta_{c}(\Lambda)(\sigma)=\left\{\begin{array}{lll}
\left(\Lambda_{c} \circ \Lambda \circ \Lambda_{c}\right)(\sigma), & \text { если } & c(\sigma) \supset c, \\
\Lambda(\sigma), & \text { если } & c(\sigma) \not \supset c .
\end{array}\right.
$$

Несложно проверить, что $\Theta_{c}(\Lambda) \in P$.

Теперь мы готовы к тому, чтобы определить отображения

$$
\Phi_{j}^{1}: U \times P \times \mathbb{Z}_{2}^{n} \rightarrow U \times P \times \mathbb{Z}_{2}^{n} .
$$

Положим

$$
\Phi_{j}^{1}\left(u\left(\sigma^{0}, \sigma^{1}, \ldots, \sigma^{n-1}\right), \Lambda, g\right)=\left(u\left(\Lambda_{c}\left(\sigma^{0}\right), \Lambda_{c}\left(\sigma^{1}\right), \ldots, \Lambda_{c}\left(\sigma^{n-1}\right)\right), \Theta_{c}(\Lambda), g+\varepsilon_{j}\right),
$$

где $c=c\left(\sigma^{j-1}\right)$.

ПРЕДЛОЖЕНИЕ 2.9. Отображения $\Phi_{j}^{1}$ сутъ корректно определенные инволющии, меняющие местами множества $V_{+} u V_{-}$и удовлетворяющие условиям 1)-3) из предложения 2.6 . 
ДоказАтельство. Очевидно, что для любых $\Lambda \in P, c \subset \mathcal{C}$ отображение $\Lambda_{c}: W_{c} \rightarrow W_{c}$ сохраняет размерности, метки симплексов и отношение включения. (Заметим, что само отображение $\Lambda: W \rightarrow W$ не сохраняет отношение включения.) Из этого следует, что отображения $\Phi_{j}^{1}$ корректно определены. Условие 2) и равенства $\Phi_{j}^{1}\left(V_{+}\right)=V_{-}$и $\Phi_{j}^{1}\left(V_{-}\right)=V_{+}$следуют из того, что симплекс $\Lambda_{c}\left(\sigma^{l}\right)$ является образом симплекса $\sigma^{l}$ при антиизоморфизме $\operatorname{star} \sigma^{j-1} \rightarrow \operatorname{star} \Lambda\left(\sigma^{j-1}\right)$. В качестве отображения $h$, удовлетворяющего условию 3 ), можно взять проекцию на последний сомножитель $U \times P \times \mathbb{Z}_{2}^{n} \rightarrow \mathbb{Z}_{2}^{n}$.

Если $c_{1} \subset c_{2} \subset \mathcal{C}$, то $W_{c_{2}} \subset W_{c_{1}}$. Поэтому для любой инволюции $\Lambda \in P$ корректно определено отображение $\Lambda_{c_{1}} \circ \Lambda_{c_{2}} \circ \Lambda_{c_{1}}: W_{c_{2}} \rightarrow W_{c_{2}}$. Для того чтобы доказать, что отображения $\Phi_{j}^{1}$ являются инволюциями и коммутируют, нам понадобятся следующие вспомогательные утверждения.

ПреДЛОЖЕНИЕ 2.10. Пустъ $c_{1} \subset c_{2} \subset \mathcal{C}, \Lambda \in P$. Тогда

$$
\begin{gathered}
\left(\Theta_{c_{2}}(\Lambda)\right)_{c_{1}}=\Lambda_{c_{1}}, \quad\left(\Theta_{c_{1}}(\Lambda)\right)_{c_{2}}=\Lambda_{c_{1}} \circ \Lambda_{c_{2}} \circ \Lambda_{c_{1}}, \\
\left(\Theta_{c_{1}}(\Lambda)\right)_{c_{2}} \circ \Lambda_{c_{1}}=\left(\Theta_{c_{2}}(\Lambda)\right)_{c_{1}} \circ \Lambda_{c_{2}} .
\end{gathered}
$$

ДокАЗАТЕЛЬСтво. Из того, что отображение $\Lambda_{c}$ сохраняет метки симплексов и отношение включения, следует, что первое равенство достаточно проверить для симплексов $\sigma$ таких, что $c(\sigma)=c_{1}$, а второе - для симплексов $\sigma$ таких, что $c(\sigma)=c_{2}$.

Если $c(\sigma)=c_{1}$, то

$$
\left(\Theta_{c_{2}}(\Lambda)\right)_{c_{1}}(\sigma)=\Theta_{c_{2}}(\Lambda)(\sigma)=\Lambda(\sigma)=\Lambda_{c_{1}}(\sigma)
$$

Если $c(\sigma)=c_{2}$, то

$$
\left(\Theta_{c_{1}}(\Lambda)\right)_{c_{2}}(\sigma)=\Theta_{c_{1}}(\Lambda)(\sigma)=\left(\Lambda_{c_{1}} \circ \Lambda \circ \Lambda_{c_{1}}\right)(\sigma)=\left(\Lambda_{c_{1}} \circ \Lambda_{c_{2}} \circ \Lambda_{c_{1}}\right)(\sigma) .
$$

Третье равенство является непосредственным следствием двух первых.

ПреДЛОЖЕНИЕ 2.11. Если $c_{1} \subset c_{2}$, mo $\Theta_{c_{1}} \circ \Theta_{c_{2}}=\Theta_{c_{2}} \circ \Theta_{c_{1}}$.

ДоказАтельство. Пусть $\Lambda \in P, \sigma \in W$. Если $c(\sigma) \not \supset c_{1}$, то

$$
\Theta_{c_{2}}\left(\Theta_{c_{1}}(\Lambda)\right)(\sigma)=\Lambda(\sigma)=\Theta_{c_{1}}\left(\Theta_{c_{2}}(\Lambda)\right)(\sigma) .
$$

Если $c(\sigma) \supset c_{1}$ и $c(\sigma) \not \supset c_{2}$, то

$$
\begin{aligned}
\Theta_{c_{1}}\left(\Theta_{c_{2}}(\Lambda)\right)(\sigma) & =\left(\left(\Theta_{c_{2}}(\Lambda)\right)_{c_{1}} \circ \Theta_{c_{2}}(\Lambda) \circ\left(\Theta_{c_{2}}(\Lambda)\right)_{c_{1}}\right)(\sigma) \\
& =\left(\Lambda_{c_{1}} \circ \Lambda \circ \Lambda_{c_{1}}\right)(\sigma) \\
\Theta_{c_{2}}\left(\Theta_{c_{1}}(\Lambda)\right)(\sigma) & =\Theta_{c_{1}}(\Lambda)(\sigma)=\left(\Lambda_{c_{1}} \circ \Lambda \circ \Lambda_{c_{1}}\right)(\sigma) .
\end{aligned}
$$

Если $c(\sigma) \supset c_{2}$, то

$$
\begin{aligned}
\Theta_{c_{1}}\left(\Theta_{c_{2}}(\Lambda)\right)(\sigma) & =\left(\left(\Theta_{c_{2}}(\Lambda)\right)_{c_{1}} \circ \Theta_{c_{2}}(\Lambda) \circ\left(\Theta_{c_{2}}(\Lambda)\right)_{c_{1}}\right)(\sigma) \\
& =\left(\Lambda_{c_{1}} \circ \Lambda_{c_{2}} \circ \Lambda \circ \Lambda_{c_{2}} \circ \Lambda_{c_{1}}\right)(\sigma), \\
\Theta_{c_{2}}\left(\Theta_{c_{1}}(\Lambda)\right)(\sigma) & =\left(\left(\Theta_{c_{1}}(\Lambda)\right)_{c_{2}} \circ \Theta_{c_{1}}(\Lambda) \circ\left(\Theta_{c_{1}}(\Lambda)\right)_{c_{2}}\right)(\sigma) \\
& =\left(\left(\Lambda_{c_{1}} \circ \Lambda_{c_{2}} \circ \Lambda_{c_{1}}\right) \circ\left(\Lambda_{c_{1}} \circ \Lambda \circ \Lambda_{c_{1}}\right) \circ\left(\Lambda_{c_{1}} \circ \Lambda_{c_{2}} \circ \Lambda_{c_{1}}\right)\right)(\sigma) \\
& =\left(\Lambda_{c_{1}} \circ \Lambda_{c_{2}} \circ \Lambda \circ \Lambda_{c_{2}} \circ \Lambda_{c_{1}}\right)(\sigma) .
\end{aligned}
$$


Из равенства $\left(\Theta_{c}(\Lambda)\right)_{c}=\Lambda_{c}$ следует, что отображения $\Phi_{j}^{1}$ являются инволюциями. Из третьего равенства предложения 2.10 и предложения 2.11 легко выводится, что эти инволюции коммутируют.

Таким образом, множество $S$ и инволюции $\Phi_{j}^{1}$ удовлетворяют условиям предложения 2.7. Значит, $\widetilde{\mathbf{Q}}(\Gamma)$ - искомое кубически клеточное псевдомногообразие. Условия согласования 1)-3) из теоремы 2.3 следуют из конструкции.

Рассмотрим теперь общий случай и сведем его к уже рассмотренному нами частному случаю. Пусть псевдомногообразие $Y$ имеет $q$ вершин. Положим $\mathcal{C}=$ $\{1,2, \ldots, q\}$. Обозначим через $\mathcal{B}$ множество биекций $\operatorname{Vert}(Y) \rightarrow \mathcal{C}$. Для каждой вершины $y$ псевдомногообразия $Y$ антиизоморфизм $\chi_{y}$ индуцирует биекцию $\operatorname{Vert}(\operatorname{star} y) \rightarrow \operatorname{Vert}(\operatorname{star} \tilde{y})$. Продолжим произвольным образом эти биекции до биекций $\varkappa_{y}: \operatorname{Vert}(Y) \rightarrow \operatorname{Vert}(Y)$ так, чтобы были выполнены условия $\varkappa_{\tilde{y}}=\varkappa_{y}^{-1}$. Положим $\bar{Y}=Y \times \mathcal{B}$. Каждую вершину $(y, \nu) \in \operatorname{Vert}(Y) \times \mathcal{B}$ мы пометим меткой $\nu(y) \in \mathcal{C}$. Определим инволюцию

$$
\bar{\lambda}: \operatorname{Vert}(\bar{Y}) \rightarrow \operatorname{Vert}(\bar{Y})
$$

по формуле

$$
\bar{\lambda}(y, \nu)=\left(\tilde{y}, \nu \circ \varkappa_{y}^{-1}\right) .
$$

Пусть $\bar{\chi}_{(y, \nu)}: \operatorname{star}(y, \nu) \rightarrow \operatorname{star} \bar{\lambda}(y, \nu)-$ антиизоморфизм, индуцированный антиизоморфизмом $\chi_{y}$. Тогда псевдомногообразие $\bar{Y}$ удовлетворяет условиям 1$)$ и 2) нашего предположения. Чтобы получить искомое псевдомногообразие $X$, нам осталось применить описанную выше конструкцию к полученному псевдомногообразию $\bar{Y}$.

2.6. Пример. Пусть $Y_{1}=Y_{2}$ - граница треугольника. Рассмотрим множество меток $\mathcal{C}=\{1,2,3\}$ и пометим вершины комплекса $Y_{1}$ метками $1,2,3$ по часовой стрелке, а вершины комплекса $Y_{2}$ - метками $1,2,3$ против часовой стрелки. Псевдомногообразие $Y=Y_{1} \sqcup Y_{2}$ удовлетворяет предположе-

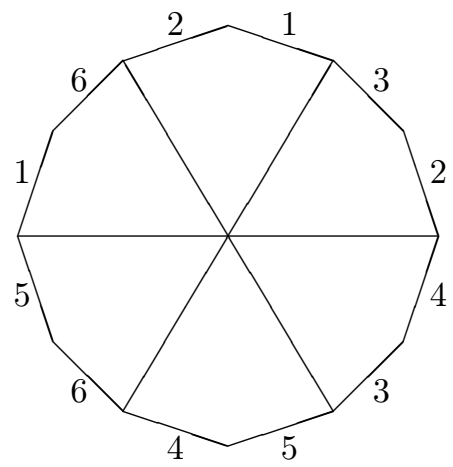

Рис. 1

нию из п. 2.5. Конструкция, описанная в п. 2.5, дает кубически клеточный комплекс, являющийся несвязным объединением двух экземпляров кубически клеточного разбиения кренделя, изображенного на рис. 1. На этом рисунке отрезки с одинаковыми номерами склеены так, чтобы получилась ориентированная поверхность. 
2.7. Оператор барицентрического подразделения. В этом пункте мы получим несколько результатов о цепном комплексе $\mathcal{T}_{*}$. В п. 2.8 эти результаты будут использованы для построения комбинаторного многообразия $K$ из теоремы 1.1. Напомним, что определение комплекса $\mathcal{T}_{*}$ было дано в $\S 1$.

Определим линейный оператор барицентрического подразделения $\beta$ : $\mathcal{T}_{*} \rightarrow \mathcal{T}_{*}$ на образующих по формуле $\beta Y=Y^{\prime}$.

ПредлОЖеНИЕ 2.12. Отображение $\beta$ является цепным по модулю элементов порядка 2 , т.е. $2(d \beta-\beta d) \xi=0$ для любого $\xi \in \mathcal{T}_{*}$.

ДокАЗАТЕЛЬСтво. Пусть $Y$ - ориентированная комбинаторная сфера. Линк каждой вершины комплекса $Y^{\prime}$, являющейся барицентром симплекса положительной размерности, обладает антиавтоморфизмом и, значит, является элементом порядка 2 в группе $\mathcal{T}_{*}$. Линк в комплексе $Y^{\prime}$ вершины $x$ комплекса $Y$ изоморфен барицентрическому подразделению линка вершины $x$ в комплексе $Y$. Поэтому

$$
d\left(Y^{\prime}\right)=\sum_{x \in \operatorname{Vert}(Y)}\left(\operatorname{link}_{Y} x\right)^{\prime}+[2] ;
$$

здесь и далее [2] - произвольные элементы порядка 2.

Существует стандартная конструкция, сопоставляющая каждому симплициальному комплексу $Y$ симплициальное разбиение $Z$ цилиндра $Y \times[0,1]$ такое, что ограничение $Z$ на нижнее основание $Y \times 0$ есть $Y$ и ограничение $Z$ на верхнее основание $Y \times 1$ есть $Y^{\prime}$ (см., например, [29]). Для каждого симплекса $\sigma$ комплекса $Y$ обозначим через $b(\sigma)$ его барицентр. Тогда $Z$ - симплициальный комплекс, составленный из симплексов, натянутых на всевозможные наборы вершин вида $\left(x_{1}, 0\right), \ldots,\left(x_{k}, 0\right),\left(b\left(\sigma_{1}\right), 1\right), \ldots,\left(b\left(\sigma_{l}\right), 1\right)$, где $x_{1}, \ldots, x_{k}$ - различные вершины комплекса $Y$, содержащиеся в некотором $(k-1)$-мерном симплексе $\tau$, и $\sigma_{1}, \ldots, \sigma_{l}$ - различные симплексы комплекса $Y$ такие, что $\tau \subset \sigma_{1} \subset \cdots \subset \sigma_{l}$.

Пусть теперь $Y$ является $(n-1)$-мерной комбинаторной сферой. Тогда $Z$ есть кусочно линейная триангуляция цилиндра $S^{n-1} \times[0,1]$ с краем, который состоит из двух компонент, одна из которых изоморфна $Y$, а вторая изоморфна $Y^{\prime}$. Заклеим эти компоненты края конусами cone $(Y)$ и cone $\left(Y^{\prime}\right)$ соответственно. В результате мы получим $n$-мерную комбинаторную сферу, для которой мы будем использовать обозначение $\widehat{Y}$.

Комбинаторная сфера $\widehat{Y}$ имеет вершины четырех типов:

1) вершина $u_{0}$ конуса cone $(Y)$;

$2)$ вершины вида $(x, 0)$, где $x$ - вершина комплекса $Y$;

3) вершины вида $(b(\sigma), 1)$, где $\sigma$ - симплекс комплекса $Y$;

4) вершина $u_{1}$ конуса cone $\left(Y^{\prime}\right)$.

Если комбинаторная сфера $Y$ ориентирована, мы выберем на комбинаторной сфере $\widehat{Y}$ такую ориентацию, что link $u_{1} \cong Y^{\prime}$ и link $u_{0} \cong-Y$.

ПРЕДЛОЖЕНИЕ 2.13. Отображение $\beta$ иепно гомотопно тождественному по модулю элементов порядка 2, т.е. существует линейное отображение $D: \mathcal{T}_{*} \rightarrow \mathcal{T}_{*}$ степени 1 такое, что

$$
\beta \xi-\xi=d D \xi+D d \xi+[2]
$$

для любого $\xi \in \mathcal{T}_{*}$. 
ДокАзАТЕЛЬСтво. Определим отображение $D$ на образующих по формуле $D Y=\widehat{Y}$. Линк каждой вершины $(x, 0)$ комбинаторной сферы $\widehat{Y}$ антиизоморфен комбинаторной сфере $\widehat{\operatorname{link}_{Y} x}$. Линк каждой вершины $(b(\sigma), 1)$ обладает антиавтоморфизмом и, следовательно, является элементом порядка 2 в группе $\mathcal{T}_{*}$. Значит,

$$
d D Y=Y^{\prime}-Y-\sum_{x \in \operatorname{Vert}(Y)} \widehat{\operatorname{link}_{Y} x}+[2]=\beta Y-Y-D d Y+[2] .
$$

СлеДСтвиЕ 2.2. Отображение $(2 \beta)_{*}: H_{*}\left(\mathcal{T}_{*}\right) \rightarrow H_{*}\left(\mathcal{T}_{*}\right)$ совпадает с умножением на 2.

2.8. Конструкция комбинаторного многообразия $K$. В этом пункте мы, опираясь на конструкцию кубически клеточного комплекса $X$ из п. 2.5, даем явную конструкцию комбинаторного многообразия $K$, удовлетворяющего условиям теоремы 1.1 .

Пусть $\left(Y_{1}, Y_{2}, \ldots, Y_{k}\right)$ - сбалансированный набор ориентированных комбинаторных сфер. Применим к нему конструкцию из п. 2.5. Полученное псевдомногообразие $X$ будет кубически клеточным комбинаторным многообразием, так как линки всех его вершин являются комбинаторными сферами. Набор линков вершин симплициального комбинаторного многообразия $X^{\prime}$ состоит из набора $\left(Y_{1}^{\prime \prime}, Y_{2}^{\prime \prime}, \ldots, Y_{k}^{\prime \prime}\right)$, взятого $r$ раз, и некоторого количества комбинаторных сфер, каждая из которых обладает антиавтоморфизмом. Значит,

$$
d\left(X^{\prime}\right)=r \sum_{i=1}^{k} Y_{i}^{\prime \prime}+[2] .
$$

Цепь $Y_{1}+Y_{2}+\cdots+Y_{k}$ является циклом в комплексе $\mathcal{T}_{*}$. Поэтому из предложений 2.12 и 2.13 следует, что

$$
\begin{aligned}
& d \sum_{i=1}^{k} \widehat{Y}_{i}=\sum_{i=1}^{k} Y_{i}^{\prime}-\sum_{i=1}^{k} Y_{i}+[2], \\
& d \sum_{i=1}^{k} \widehat{Y_{i}^{\prime}}=\sum_{i=1}^{k} Y_{i}^{\prime \prime}-\sum_{i=1}^{k} Y_{i}^{\prime}+[2] .
\end{aligned}
$$

Положим

$$
K=X^{\prime} \sqcup X^{\prime} \sqcup\left(\left(-\widehat{Y}_{1}\right) \sqcup \cdots \sqcup\left(-\widehat{Y}_{k}\right)\right)^{\sqcup 2 r} \sqcup\left(\left(-\widehat{Y_{1}^{\prime}}\right) \sqcup \cdots \sqcup\left(-\widehat{Y_{k}^{\prime}}\right)\right)^{\sqcup 2 r} .
$$

Тогда

$$
d K=2 r \sum_{i=1}^{k} Y_{i} .
$$

Значит, $K$ - искомое комбинаторное многообразие.

2.9. Малые накрытия. Малые накрытия представляют собой $\mathbb{Z}_{2}$-аналоги квазиторических многообразий (см. [4], [5]). Малым накрытием называется гладкое многообразие $M^{n}$ с локально стандартным действием группы $\mathbb{Z}_{2}^{n}$ такое, что $M^{n} / \mathbb{Z}_{2}^{n}=P^{n}$ - простой многогранник. (Действие называется локально 
стандартным, если оно локально моделируется стандартным действием группы $\mathbb{Z}_{2}^{n}$ отражениями на $\mathbb{R}^{n}$.) М. Дэвис и Т. Янушкевич в 1991 г. доказали [4], что каждое малое накрытие получается с помощью некоторой стандартной конструкции. В этом пункте мы изложим эту конструкцию Дэвиса-Янушкевича и покажем, как она связана с нашей конструкцией, описанной в п. 2.5.

Пусть $P$ - n-мерный простой многогранник с $m$ гипергранями $F_{1}, F_{2}, \ldots, F_{m}$. Пусть $Y$ - граница симплициального многогранника, двойственного многограннику $P$. Пусть $\left(a_{1}, a_{2}, \ldots, a_{m}\right)$ - базис группы $\mathbb{Z}_{2}^{m}$. Характеристической функиией называется произвольный гомоморфизм $\lambda: \mathbb{Z}_{2}^{m} \rightarrow \mathbb{Z}_{2}^{n}$ такой, что элементы $\lambda\left(a_{i_{1}}\right), \lambda\left(a_{i_{2}}\right), \ldots, \lambda\left(a_{i_{k}}\right)$ линейно независимы всякий раз, когда пересечение $F_{i_{1}} \cap F_{i_{2}} \cap \cdots \cap F_{i_{k}}$ не пусто. Пусть $F$ - грань многогранника $P$, являющаяся пересечением гиперграней $F_{i_{1}}, F_{i_{2}}, \ldots, F_{i_{k}}$. Обозначим через $G(F) \subset \mathbb{Z}_{2}^{n}$ подгруппу, порожденную элементами $\lambda\left(a_{i_{1}}\right), \lambda\left(a_{i_{2}}\right), \ldots, \lambda\left(a_{i_{k}}\right)$. Для каждой точки $x \in P$ обозначим через $F(x)$ (единственную) грань многогранника $P$, содержащую точку $x$ в своей относительной внутренности. Положим,

$$
M_{P, \lambda}=P \times \mathbb{Z}_{2}^{n} / \sim
$$

где $\left(x_{1}, g_{1}\right) \sim\left(x_{2}, g_{2}\right)$ тогда и только тогда, когда $x_{1}=x_{2}$ и $g_{1}^{-1} g_{2} \in G\left(F\left(x_{1}\right)\right)$. Тогда $M_{P, \lambda}$ - многообразие, разбитое на клетки, каждая из которых изоморфна многограннику $P$. Рассмотрим клеточное разбиение $X_{P, \lambda}$, двойственное полученному разбиению многообразия $M_{P, \lambda}$. Несложно проверить, что $X_{P, \lambda}-$ кубическое комбинаторное многообразие, линк каждой вершины которого изоморфен комплексу $Y$. K сожалению, конструкция многообразия $X_{P, \lambda}$ не дает ответа на вопрос 1.2 , так как ровно половина из $2^{n}$ вершин комплекса $X_{P, \lambda}$ имеют линки, изоморфные комбинаторной сфере $Y$ с сохранением ориентации, а остальные - с обращением ориентации.

Покажем теперь, что в некотором частном случае кубическое разбиение $X_{P, \lambda}$ может быть получено с помощью некоторого аналога предложения 2.7. Мы будем рассматривать случай так называемых многообразий, индуцированных из линейной модели (см. [4]). Малое накрытие $M_{P, \lambda}$ называется многообразием, индуиированным из линейной модели, если функция $\lambda$ переводит каждый вектор $a_{i}$ в некоторый базисный вектор из наперед фиксированного базиса группы $\mathbb{Z}_{2}^{n}$. Такая характеристическая функция задает правильную раскраску вершин комбинаторной сферы $Y$ в $n$ цветов. Пусть $\Upsilon$ - соответствующий однородный граф степени $n, U-$ множество его вершин, $\Phi_{j}-$ инволюции, задающие граф $\Upsilon$. Будем действовать по аналогии с предложением 2.7: для некоторого конечного множества $S$ положим

$$
V=U \times S, \quad \Phi_{j}^{0}(u, s)=\left(\Phi_{j}(u), s\right)
$$

и будем искать инволюции $\Phi_{j}^{1}: V \rightarrow V$, удовлетворяющие условиям 1)-3) из предложения 2.6. Единственное отличие: мы не хотим следить за ориентациями линков вершин получающегося комплекса, поэтому мы не будем налагать на инволюции $\Phi_{j}^{1}$ ограничений $\Phi_{j}^{1}\left(V_{+}\right)=V_{-}$и $\Phi_{j}^{1}\left(V_{-}\right)=V_{+}$. В этом случае все трудности, преодолению которых посвящен п. 2.5, можно легко обойти. Положим $S=\mathbb{Z}_{2}^{n}$ и

$$
\Phi_{j}^{1}(u, s)=\left(u, s+\varepsilon_{j}\right)
$$


где $\left(\varepsilon_{1}, \varepsilon_{2}, \ldots, \varepsilon_{n}\right)$ - базис группы $\mathbb{Z}_{2}^{n}$. Полученный однородный граф степени $2 n$ мы обозначим через $\Gamma$. Несложно проверить, что $\widetilde{\mathbf{Q}}(\Gamma) \cong X_{P, \lambda}$.

\section{§ 3. Кобордизмы многообразий с особенностями}

3.1. Классы псевдомногообразий. Напомним, что под псевдомногообразием мы всегда понимаем нормальное псевдомногообразие (см. п. 2.1). В этом параграфе мы, если не оговорено противное, всегда будем понимать под псевдомногообразием симплициальное псевдомногообразие. Пусть С - класс ориентированных псевдомногообразий, удовлетворяющий следующим аксиомам.

Аксиома I. Нулвмерное псевдомногообразие принадлежит классу $\mathrm{C}$ тогда и толъко тогда, когда оно является нульмерной сферой. Все псевдомногообразия положительной размерности, принадлежащие классу С, являются связными.

Аксиома II. Если псевдомногообразие $Y$ принадлежит классу С, то любое псевдомногообразие, кусочно линейно гомеоморфное псевдомногообразию $Y c$ сохранением или с обращением ориентации, также принадлежит классу С.

Аксиома III. Если п-мерное псевдомногообразие $Y$ принадлежит классу C, $\sigma$ - симплекс псевдомногообразия $Y$ такой, что $\operatorname{dim} \sigma<n$, то псевдомногообразие link $\sigma$ принадлежит классу $\mathrm{C}$.

Аксиома IV. Если псевдомногообразие $Y$ принадлежит классу $\mathrm{C}$, то неприведенная надстройка $\Sigma Y$ также принадлежит классу C.

Обозначим через РМ класс, состоящий из нульмерных сфер и всех связных ориентированных псевдомногообразий положительной размерности. Это наибольший класс, удовлетворяющий аксиомам I-IV. C другой стороны, из аксиом сразу следует, что любая комбинаторная сфера принадлежит классу С. Поэтому наименьшим классом, удовлетворяющим аксиомам I-IV, является класс CS всех ориентированных комбинаторных сфер.

Приведем примеры классов, удовлетворяющих аксиомам I-IV.

1. Пусть $P_{1}, P_{2}, \ldots$ - конечная или бесконечная последовательность комбинаторных многообразий. Класс $\mathrm{C}\left(P_{1}, P_{2}, \ldots\right)$ состоит из всех комбинаторных сфер и всех псевдомногообразий $X$, для которых существуют натуральные числа $i_{1}<i_{2}<\cdots<i_{k}$ и неотрицательное целое число $q$ такие, что псевдомногообразие $X$ кусочно линейно гомеоморфно (с сохранением или с обращением ориентации) $q$-кратной надстройке над джойном $P_{i_{1}} * P_{i_{2}} * \cdots * P_{i_{k}}$.

2. Симплициальный комплекс называется $n$-мерным симплициальным гомологическим многообразием, если линк каждого его $k$-мерного симплекса имеет гомологии $(n-k-1)$-мерной сферы. Симпличиальной гомологической сферой называется симплициальное гомологическое многообразие, имеющее гомологии сферы. Класс HS всех ориентированных симплициальных гомологических сфер удовлетворяет аксиомам I-IV. Рассматривая вместо целочисленных гомологий гомологии с коэффициентами в абелевой группе $A$, мы аналогично получаем класс $\operatorname{HS}(A)$ всех ориентированных симплициальных $A$-гомологических сфер. Этот класс также удовлетворяет аксиомам I-IV. 
3. В [30], [19] Дж. Чигер построил $L_{2}$-теорию Ходжа для так называемых псевдомногообразий с пренебрежимой границей. На каждом $n$-мерном псевдомногообразии $X$ можно ввести локально плоскую метрику, ограничение которой на каждый симплекс совпадает с евклидовой метрикой на правильном симплексе с ребром 1. Тогда $X \backslash X^{n-2}$ - неполное гладкое риманово многообразие, где $X^{n-2}-(n-2)$-мерный остов комплекса $X$. Обозначим через $H_{(2)}^{*}\left(X \backslash X^{n-2}\right)$ кольцо $L_{2}$-когомологий этого многообразия (см. [31]). По определению положим $H_{(2)}^{*}(X)=H_{(2)}^{*}\left(X \backslash X^{n-2}\right)$. Определенное таким образом кольцо $H_{(2)}^{*}(X)$ инвариантно относительно кусочно линейных гомеоморфизмов [30]. Псевдомногообразие $X$ называется псевдомногообразием с пренебрежимой гранищей, если для любого его симплекса $\sigma$ такого, что $\operatorname{dim} \operatorname{link} \sigma=2 k>0$, мы имеем $H_{(2)}^{k}(\operatorname{link} \sigma)=0$. Важность этого условия заключается в том, что оно обеспечивает сопряженность сильных замыканий операторов $d$ и $\delta$ в пространстве $L_{2}$-форм на многообразии $X \backslash X^{n-2}$ (см. [30], [31]). Обозначим через Ch класс, состоящий из нульмерных сфер, всех нечетномерных псевдомногообразий с пренебрежимой границей и всех четномерных псевдомногообразий $X$ с пренебрежимой границей таких, что $H_{(2)}^{m}(X)=0$, где $\operatorname{dim} X=2 m$. Непосредственно проверяется, что класс Ch удовлетворяет аксиомам I-IV.

В работе [2] автором был построен цепной комплекс $\mathcal{T}_{*}$ ориентированных комбинаторных сфер (см. определение в 1 настоящей работы). Дадим обобщение этой конструкции для случая произвольного класса С.

Для простоты мы будем обозначать класс изоморфизма псевдомногообразия той же буквой, что и само псевдомногообразие, и иногда вообще не будем различать псевдомногообразие и его класс изоморфизма.

Фиксируем некоторое натуральное число $n$. Рассмотрим свободную абелеву группу, порожденную всеми классами изоморфизма ориентированных $(n-1)$-мерных псевдомногообразий из класса С. Профакторизуем эту группу по соотношениям $Y+(-Y)=0$, где $-Y$ - псевдомногообразие $Y$ с обращенной ориентацией. Полученную группу обозначим через $\mathcal{T}_{n}^{\mathrm{C}}$. Таким образом, $\mathcal{T}_{n}^{\mathrm{C}}-$ абелева группа, разлагающаяся в прямую сумму слагаемых, каждое из которых изоморфно $\mathbb{Z}$ или $\mathbb{Z}_{2}$. Слагаемые $\mathbb{Z}$ соответствуют псевдомногообразиям, не обладающим антиавтоморфизмами, слагаемые $\mathbb{Z}_{2}$ - псевдомногообразиям, обладающим антиавтоморфизмами. Удобно считать, что $\mathcal{T}_{0}^{\mathrm{C}} \cong \mathbb{Z}-$ свободная группа, порожденная элементом $\varnothing$.

Определим дифференциал

$$
d: \mathcal{T}_{n}^{\mathrm{C}} \rightarrow \mathcal{T}_{n-1}^{\mathrm{C}}
$$

по формуле

$$
d Y=\sum_{y \in \operatorname{Vert}(Y)} \operatorname{link} y,
$$

где каждое псевдомногообразие link $y$ берется с ориентацией, индуцированной ориентацией псевдомногообразия $Y$. (Дифференциал $d: \mathcal{T}_{1}^{\mathrm{C}} \rightarrow \mathcal{T}_{0}^{\mathrm{C}}$ тривиален.) Несложно проверить, что $d^{2}=0$. Таким образом, $\mathcal{T}_{*}^{\mathrm{C}}$ - цепной комплекс.

Для произвольной абелевой группы $A$ введем обозначение

$$
\mathcal{T}_{\mathrm{C}}^{*}(A)=\operatorname{Hom}\left(\mathcal{T}_{*}^{\mathrm{C}}, A\right) .
$$


Элементами группы $\mathcal{T}_{\mathrm{C}}^{n}(A)$ являются $A$-значные функции $f$ на множестве классов изоморфизма $(n-1)$-мерных псевдомногообразий из класса С, удовлетворяющие условию $f(-Y)=-f(Y)$. При этом кограничный оператор

$$
\delta: \mathcal{T}_{\mathrm{C}}^{n}(A) \rightarrow \mathcal{T}_{\mathrm{C}}^{n+1}(A)
$$

задается по формуле

$$
(\delta f)(Y)=(-1)^{n} \sum_{y \in \operatorname{Vert}(Y)} f(\operatorname{link} y)
$$

Предположим теперь, что класс С удовлетворяет следующей аксиоме мультипликативности.

Аксиома V. Если псевдомногообразия $Y_{1}$ и $Y_{2}$ принадлежат классу $\mathrm{C}$, то их джойн $Y_{1} * Y_{2}$ тоже принадлежит классу C.

Тогда операция взятия джойна задает билинейное умножение

$$
*: \mathcal{T}_{m}^{\mathrm{C}} \times \mathcal{T}_{n}^{\mathrm{C}} \rightarrow \mathcal{T}_{m+n}^{\mathrm{C}}
$$

которое превращает градуированную группу $\mathcal{T}_{*}^{\mathrm{C}}$ в коммутативное градуированное кольцо. Имеет место тождество Лейбница

$$
d\left(Y_{1} * Y_{2}\right)=d Y_{1} * Y_{2}+(-1)^{m} Y_{1} * d Y_{2}
$$

Поэтому $H_{*}\left(\mathcal{T}_{*}^{\mathrm{C}}\right)$ - коммутативное градуированное кольцо.

Отметим, что классы $\mathrm{PM}, \mathrm{CS}, \mathrm{HS}(A)$, Ch удовлетворяют аксиоме $\mathrm{V}$, классы $\mathrm{C}\left(P_{1}, P_{2}, \ldots\right)$, вообще говоря, не удовлетворяют ей.

3.2. Группы С-кобордизмов. Пусть С - класс псевдомногообразий, удовлетворяющий аксиомам I-IV. Многообразием с особенностями из класса С мы будем называть псевдомногообразие, линки всех вершин которого принадлежат классу С. В частности, псевдомногообразия с особенностями из класса РМ это все нормальные псевдомногообразия, из класса CS - все комбинаторные многообразия, из класса HS - все симплициальные гомологические многообразия, из класса $\mathrm{Ch}$ - все псевдомногообразия с пренебрежимой границей. Класс всех ориентированных (не обязательно связных) многообразий с особенностями из класса $\mathrm{C}$ мы обозначим через $\widetilde{\mathrm{C}}$. Из аксиомы III следует, что $\mathrm{C} \subset \widetilde{\mathrm{C}}$.

Пусть $K=K_{1} \sqcup \cdots \sqcup K_{k}$ и $L=L_{1} \sqcup \cdots \sqcup L_{l}-n$-мерные многообразия с особенностями из класса $\mathrm{C}$ (комплексы $K_{i}$ и $L_{i}$ связны). Будем говорить, что многообразия $K$ и $L$ С-кобордантны, если существует замкнутое ориентированное симплициальное псевдомногообразие $Z$ с вершинами $x_{1}, \ldots, x_{k}$, $y_{1}, \ldots, y_{l}, z_{1}, \ldots, z_{m}$ такими, что $\operatorname{link} x_{i} \cong K_{i}$ при $i=1, \ldots, k, \operatorname{link} y_{i} \cong-L_{i}$ при $i=1, \ldots, l$ и link $z_{i} \in \mathrm{C}$ при $i=1, \ldots, m$. Обозначим через $\Omega_{n}^{\mathrm{C}}$ соответствующую полугруппу кобордизмов. Поскольку класс С удовлетворяет аксиоме IV, псевдомногообразие $\Sigma K$ осуществляет С-кобордизм между псевдомногообразием $K \sqcup(-K)$ и пустым псевдомногообразием. Поэтому полугруппа $\Omega_{n}^{\mathrm{C}}$ является группой. Имеем следующее: $\Omega_{0}^{\mathrm{C}} \cong \mathbb{Z}-$ группа, порожденная классом кобордизмов точки. 
ЗАмЕчАниЕ 3.1. Приведенное выше определение отношения кобордантности имеет простой геометрический смысл: если в псевдомногообразии $Z$ вырезать регулярные окрестности вершин $x_{1}, \ldots, x_{k}, y_{1}, \ldots, y_{l}$, мы получим многообразие с особенностями из класса С с краем, изоморфным псевдомногообразию $K \sqcup(-L)$.

Чтобы введенное отношение С-кобордантности было геометрически содержательным, нам нужно доказать следующее предложение.

ПрЕДЛОЖЕНИЕ 3.1. Пусть $K_{1}$ и $K_{2}$ - кусочно линейно гомеоморфные с сохранением ориентации многообразия с особенностями из класса С. Тогда многообразия $K_{1}$ и $K_{2}$ являются С-кобордантными.

ДоКАЗАТЕЛЬСтво. Без ограничения общности можно считать, что псевдомногообразия $K_{1}$ и $K_{2}$ связны. Рассмотрим кусочно линейную триангуляцию $Z$ полиэдра $\Sigma K_{1}$ такую, что линки вершин надстройки $x$ и $y$ изоморфны соответственно псевдомногообразиям $K_{1}$ и $\left(-K_{2}\right)$. Пусть $z$ - произвольная вершина триангуляции $Z$, отличная от $x$ и $y$. Рассмотрим симплекс $\sigma$ комплекса $K_{1}$, в относительной внутренности которого лежит образ точки $z$ при естественной проекции $\Sigma K_{1} \backslash\{x, y\} \rightarrow K_{1}$. (Относительной внутренностью вершины считается сама эта вершина.) Тогда линк вершины $z$ в триангуляции $Z$ кусочно линейно гомеоморфен полиэдру $\Sigma\left(\partial \sigma * \operatorname{link}_{K_{1}} \sigma\right)$. Следовательно, link $z \in \mathrm{C}$. Таким образом, псевдомногообразие $Z$ осуществляет С-кобордизм между псевдомногообразиями $K_{1}$ и $K_{2}$.

Если класс С удовлетворяет аксиоме $\mathrm{V}$, в градуированной группе $\Omega_{*}^{\mathrm{C}}$ можно ввести кольцевую структуру. Прямое произведение двух псевдомногообразий $K_{1}, K_{2} \in \widetilde{\mathrm{C}}$ конечно же не является симплициальным псевдомногообразием. Однако мы можем взять произвольную кусочно линейную триангуляцию $K$ полиэдра $K_{1} \times K_{2}$. Пусть $y$ - произвольная вершина триангуляции $K$ и $y_{i}$, $i=1,2$, - ее образы при проекциях $K_{1} \times K_{2} \rightarrow K_{i}$. Пусть $\sigma_{i}-$ симплекс триангуляции $K_{i}$, в относительной внутренности которого лежит точка $y_{i}$. Несложно проверить, что линк вершины $y$ в комплексе $K$ кусочно линейно гомеоморфен комплексу

$$
\partial \sigma_{1} * \operatorname{link}_{K_{1}} \sigma_{1} * \partial \sigma_{2} * \operatorname{link}_{K_{2}} \sigma_{2}
$$

и, значит, принадлежит классу С. Следовательно, псевдомногообразие $K$ принадлежит классу $\widetilde{\mathrm{C}}$. По предложению 3.1 его класс С-кобордизмов $[K]$ не зависит от произвольного выбора триангуляции $K$. Положим $\left[K_{1}\right]\left[K_{2}\right]=[K]$. Несложно проверить, что таким образом мы получаем корректно определенную операцию умножения в градуированной группе $\Omega_{*}^{\mathrm{C}}$, относительно которой она является коммутативным ассоциативным градуированным кольцом.

Непосредственно из определения следует, что каждое псевдомногообразие, принадлежащее классу С, представляет нулевой класс кобордизмов в $\Omega_{*}^{\mathrm{C}}$. В частности, имеет место следующее предложение.

ПрЕДЛОЖЕНИЕ 3.2. Имеем $\Omega_{n}^{\mathrm{PM}}=0$ nрu $n>0$.

Кольцо $\Omega_{*}^{\mathrm{CS}}$ совпадает с кольцом $\Omega_{*}^{\mathrm{SPL}}$ ориентированных кусочно линейных кобордизмов. Как известно, кольцо $\Omega_{*}^{\mathrm{SPL}} \otimes \mathbb{Q} \cong \Omega_{*}^{\mathrm{SO}} \otimes \mathbb{Q}$ является кольцом 
полиномов с образующими во всех размерностях, кратных четырем, и

$$
\operatorname{Hom}\left(\Omega_{*}^{\mathrm{SPL}}, \mathbb{Q}\right) \cong \operatorname{Hom}\left(\Omega_{*}^{\mathrm{SO}}, \mathbb{Q}\right) \cong H^{*}(\mathrm{BO} ; \mathbb{Q}) \cong \mathbb{Q}\left[p_{1}, p_{2}, \ldots\right], \quad \operatorname{deg} p_{i}=4 i .
$$

3.3. Гомоморфизм $d_{*}: \Omega_{*}^{\mathrm{C}} \rightarrow H_{*}\left(\mathcal{T}_{*}^{\mathrm{C}}\right)$. Имеет место следующий аналог теорем 1.1 и 2.1 .

ТЕОРема 3.1. Пусть $\left(Y_{1}, Y_{2}, \ldots, Y_{k}\right)$ - сбалансированный набор ориентированных $(n-1)$-мерных псевдомногообразий из класса С. Тогда существует ориентированное $n$-мерное (симплициальное) многообразие $K$ с особенностями из класса С, набор линков вершин которого совпадает с точностью до изоморфизма с набором

$$
(\underbrace{Y_{1}, \ldots, Y_{1}}_{r}, \underbrace{Y_{2}, \ldots, Y_{2}}_{r}, \ldots, \underbrace{Y_{k}, \ldots, Y_{k}}_{r}, Z_{1}, Z_{2}, \ldots, Z_{l},-Z_{1},-Z_{2}, \ldots,-Z_{l})
$$

для некоторого натурального числа $r$ u некоторых ориентированных $(n-1)$-мерных псевдомногообразий $Z_{1}, Z_{2}, \ldots, Z_{l}$ из класса $\mathrm{C}$.

Конструкция псевдомногообразия $K$ полностью повторяет конструкцию из $\S 2$. Действительно, все результаты из пп. 2.7, 2.8 остаются в силе, если везде заменить слова "комбинаторная сфера" на "псевдомногообразие из класса C", "комбинаторное многообразие" на "многообразие с особенностями из класса С" и "цепной комплекс $\mathcal{T}_{*}$ " на "цепной комплекс $\mathcal{T}_{*}^{\mathrm{C}}$ ". В частности, имеется оператор барицентрического подразделения $\beta: \mathcal{T}_{*}^{\mathrm{C}} \rightarrow \mathcal{T}_{*}^{\mathrm{C}}$, являющийся цепным отображением по модулю элементов порядка 2 и цепно гомотопный тождественному по модулю элементов порядка 2. Тот факт, что построенное псевдомногообразие $K$ является симплициальным многообразием с особенностями из класса $\mathrm{C}$, легко выводится из аксиом.

Пусть $K$ - ориентированное $n$-мерное многообразие с особенностями из класса С. Обозначим через $d K$ элемент группы $\mathcal{T}_{*}^{\mathrm{C}}$, определяемый по формуле

$$
d K=\sum_{y \in \operatorname{Vert}(K)} \operatorname{link} y
$$

ПРЕДЛОЖЕНИЕ 3.3. Соответствие d индуиирует корректно определенный аддитивнъй гомоморфизм $d_{*}: \Omega_{*}^{\mathrm{C}} \rightarrow H_{*}\left(\mathcal{T}_{*}^{\mathrm{C}}\right)$.

ДоказатеЛЬСтво. Очевидно, что $d d K=0$, т. е. $d K-$ цикл в цепном комплексе $\mathcal{T}_{*}^{\mathrm{C}}$. Если псевдомногообразие $K$ принадлежит классу С, то автоматически $d K$ - граница в $\mathcal{T}_{*}^{\mathrm{C}}$. Пусть $Z-\mathrm{C}$-кобордизм между псевдомногообразиями $K=K_{1} \sqcup \cdots \sqcup K_{k}$ и $L=L_{1} \sqcup \cdots \sqcup L_{l}$. Будем использовать те же обозначения для вершин комплекса $Z$, что и ранее. Тогда

$$
\sum_{i=1}^{k} d \operatorname{link} x_{i}+\sum_{i=1}^{l} d \operatorname{link} y_{i}+\sum_{i=1}^{m} d \operatorname{link} z_{i}=0 .
$$

Следовательно,

$$
d K-d L=\sum_{i=1}^{k} d K_{i}-\sum_{i=1}^{l} d L_{i}=-\sum_{i=1}^{m} d \operatorname{link} z_{i}
$$

что есть граница в комплексе $\mathcal{T}_{*}^{\mathrm{C}}$, так как $\operatorname{link} z_{i} \in \mathrm{C}, i=1, \ldots, m$. 
Теорема 3.2. Если класс C удовлетворяет аксиомам I-IV, то ядро и коядро гомоморфизма $d_{*}: \Omega_{*}^{\mathrm{C}} \rightarrow H_{*}\left(\mathcal{T}_{*}^{\mathrm{C}}\right)$ являются группами кручения. Если класс $\mathrm{C}$ удовлетворяет также аксиоме $\mathrm{V}$, то гомоморфизм $d_{*}$ мультипликативен с точностью до элементов порядка 2.

Автору не известно, является ли отображение $d_{*}$ мультипликативным.

СлЕДСТВИЕ 3.1. Если класс С удовлетворяет аксиомам I-V, то гомоморфизм

$$
d_{*} \otimes \mathbb{Q}: \Omega_{*}^{\mathrm{C}} \otimes \mathbb{Q} \rightarrow H_{*}\left(\mathcal{T}_{*}^{\mathrm{C}}\right) \otimes \mathbb{Q}
$$

является мультипликативным изоморфизмом. В частности, гомоморфизмы

$$
\begin{aligned}
& d_{*} \otimes \mathbb{Q}: \Omega_{*}^{\mathrm{SPL}} \otimes \mathbb{Q} \rightarrow H_{*}\left(\mathcal{T}_{*}^{\mathrm{CS}}\right) \otimes \mathbb{Q}, \\
& d_{*} \otimes \mathbb{Q}: \Omega_{*}^{\mathrm{HS}} \otimes \mathbb{Q} \rightarrow H_{*}\left(\mathcal{T}_{*}^{\mathrm{HS}}\right) \otimes \mathbb{Q}
\end{aligned}
$$

являются мультипликативными изоморфизмами.

СлеДСТВИЕ 3.2. Группы $H_{n}\left(\mathcal{T}_{*}^{\mathrm{PM}}\right)$ являются группами кручения при $n>0$. Таким образом, $H_{n}\left(\mathcal{T}_{*}^{\mathrm{PM}} \otimes \mathbb{Q}\right)=0 u H^{n}\left(\mathcal{T}_{\mathrm{PM}}^{*}(\mathbb{Q})\right)=0$ nри $n>0$.

ДоКАЗАТЕЛЬСТво ТЕОРЕМЫ 3.2. Из теоремы 3.1 сразу следует, что коядро гомоморфизма $d_{*}$ - группа кручения. Утверждение о том, что ядро гомоморфизма $d_{*}$ является группой кручения, эквивалентно следующему предложению.

ПРеДЛОЖЕНИЕ 3.4. Пусть набор $\left(K_{1}, K_{2}, \ldots, K_{k}\right)$ связных псевдомногообразий из класса $\widetilde{\mathrm{C}}$ сбалансирован. Тогда для некоторого натуралъного числа $r$ несвязное объединение $r$ экземпляров псевдомногообразия $K=K_{1} \sqcup \cdots \sqcup K_{k}$ С-кобордантно нулю.

ДокАЗАтельство. Применим теорему 2.2 к сбалансированному набору $\left(K_{1}, K_{2}, \ldots, K_{k}\right)$ псевдомногообразий. Пусть $X$ - полученное кубически клеточное псевдомногообразие. Линк любого симплекса каждого из комплексов $K_{i}^{\prime}$ принадлежит классу С. Следовательно, линки всех вершин псевдомногообразия $X^{\prime}$, не являющихся вершинами псевдомногообразия $X$, принадлежат классу С. Если $x$ - вершина комплекса $X$, то ее линк в комплексе $X^{\prime}$ изоморфен одному из псевдомногообразий $K_{i}^{\prime \prime}$, причем каждое из псевдомногообразий $K_{i}^{\prime \prime}$ возникает в качестве линка вершины комплекса $X$ ровно $r$ раз. Значит, псевдомногообразие $X^{\prime}$ осуществляет С-кобордизм между псевдомногообразием $\left(K^{\prime \prime}\right)^{\sqcup r}$ и пустым псевдомногообразием. Осталось заметить, что по предложению 3.1 псевдомногообразие $K^{\prime \prime}$ С-кобордантно псевдомногообразию $K$.

Докажем теперь, что $d_{*}$ - мультипликативный гомоморфизм с точностью до элементов порядка 2 . Пусть $K_{1}, K_{2}$ - два псевдомногообразия из класса С Рассмотрим произведение $K_{1} \times K_{2}$. Это комплекс, склеенный из клеток, каждая из которых является произведением симплексов. Тогда $K=\left(K_{1} \times K_{2}\right)^{\prime}-$ симплициальное псевдомногообразие из класса $\widetilde{\mathrm{C}}$ и $[K]=\left[K_{1}\right]\left[K_{2}\right]$ в кольце $\Omega_{*}^{\mathrm{C}}$. Каждая вершина псевдомногообразия $K$ имеет вид $\left(b\left(\sigma_{1}\right), b\left(\sigma_{2}\right)\right)$, где $\sigma_{1}$ - симплекс комплекса $K_{1}$ и $\sigma_{2}$ - симплекс комплекса $K_{2}$. При этом

$$
\operatorname{link}_{K}\left(b\left(\sigma_{1}\right), b\left(\sigma_{2}\right)\right) \cong(-1)^{\operatorname{codim} \sigma_{1} \operatorname{dim} \sigma_{2}}\left(\partial\left(\sigma_{1} \times \sigma_{2}\right)\right)^{\prime} *\left(\operatorname{link}_{K_{1}} \sigma_{1} * \operatorname{link}_{K_{2}} \sigma_{2}\right)^{\prime} .
$$


Если $\operatorname{dim} \sigma_{1} \neq 0$ или $\operatorname{dim} \sigma_{2} \neq 0$, то комплекс $\left(\partial\left(\sigma_{1} \times \sigma_{2}\right)\right)^{\prime}$ обладает антиавтоморфизмом. Значит, псевдомногообразие $\operatorname{link}_{K}\left(b\left(\sigma_{1}\right), b\left(\sigma_{2}\right)\right)$ обладает антиавтоморфизмом и, следовательно, является элементом порядка 2 в группе $\mathcal{T}_{*}^{\mathrm{C}}$. Поэтому

$$
d K=\sum_{\substack{y_{1} \in \operatorname{Vert}\left(K_{1}\right) \\ y_{2} \in \operatorname{Vert}\left(K_{2}\right)}}\left(\operatorname{link}_{K_{1}} y_{1} * \operatorname{link}_{K_{2}} y_{2}\right)^{\prime}+[2]=\beta\left(\left(d K_{1}\right) *\left(d K_{2}\right)\right)+[2] .
$$

Значит,

$$
2 d_{*}\left(\left[K_{1}\right]\left[K_{2}\right]\right)=(2 \beta)_{*}\left(\left(d_{*}\left[K_{1}\right]\right) *\left(d_{*}\left[K_{2}\right]\right)\right)=2\left(d_{*}\left[K_{1}\right]\right) *\left(d_{*}\left[K_{2}\right]\right) .
$$

3.4. Локальные формулы для инвариантов С-кобордизмов. $А \partial \partial u$ тивным инвариантом $n$-мерных С-кобордизмов мы будем называть произвольный аддитивный гомоморфизм $q: \Omega_{n}^{\mathrm{C}} \rightarrow A$, где $A$ - абелева группа. Значение инварианта $q$ на классе $\mathrm{C}$-кобордизмов многообразия $K$ мы будем обозначать через $q(K)$.

ОПРЕДЕЛЕНИЕ 3.1. Локалъной формулой для инварианта $q$ мы будем называть аддитивный гомоморфизм $f: \mathcal{T}_{n}^{\mathrm{C}} \rightarrow A$ такой, что

$$
q(K)=\sum_{y \in \operatorname{Vert}(K)} f(\operatorname{link} y)
$$

для любого ориентированного $n$-мерного многообразия $K$ с особенностями из класса С.

Из предложения 3.3 легко выводится следующее утверждение.

СледСТВие 3.3. Аддитивный гомоморфизм $f: \mathcal{T}_{n}^{\mathrm{C}} \rightarrow$ А является локальной формулой для некоторого аддитивного инварианта С-кобордизмов тогда и только тогда, когда он является коциклом в комплексе $\mathcal{T}_{\mathrm{C}}^{*}(A)=$ $\operatorname{Hom}\left(\mathcal{T}_{*}^{\mathrm{C}}, A\right)$. Когомологичные коцикль $f$ задают один и тот же аддитивный инвариант С-кобордизмов.

Таким образом, мы получаем аддитивный гомоморфизм

$$
\delta^{*}: H^{*}\left(\mathcal{T}_{\mathrm{C}}^{*}(A)\right) \rightarrow \operatorname{Hom}\left(\Omega_{*}^{\mathrm{C}}, A\right),
$$

сопряженный гомоморфизму $d_{*}$. Из следствия 3.1 вытекает такое утверждение.

СлЕДСТВИЕ 3.4. Если класс С удовлетворяет аксиомам I-IV, то гомоморфизм $\delta^{*}: H^{*}\left(\mathcal{T}_{\mathrm{C}}^{*}(\mathbb{Q})\right) \rightarrow \operatorname{Hom}\left(\Omega_{*}^{\mathrm{C}}, \mathbb{Q}\right)$ является изоморфизмом. Таким образом, для каждого рационального аддитивного инварианта С-кобордизмов имеется локальная формула, единственная с точностью до кограницы комплекса $\mathcal{T}_{\mathrm{C}}^{*}(\mathbb{Q})$.

Рассмотрим более подробно случай $\mathrm{C}=\mathrm{CS}$. Рациональные аддитивные инварианты ориентированных кусочно линейных кобордизмов - это в точности линейные комбинации чисел Понтрягина. Согласно следствию 3.4 имеем

$$
H^{*}\left(\mathcal{T}_{\mathrm{CS}}^{*}(\mathbb{Q})\right) \cong \operatorname{Hom}\left(\Omega_{*}^{\mathrm{SPL}}, \mathbb{Q}\right) \cong \mathbb{Q}\left[p_{1}, p_{2}, \ldots\right], \quad \operatorname{deg} p_{i}=4 i
$$


Это утверждение является одним из основных результатов работы [2]. Приведенное в [2] доказательство основано на использовании теории блочных расслоений и технически довольно сложно. В настоящей статье мы даем более прямое и чисто комбинаторное доказательство следствия 3.4.

В случае произвольной абелевой группы $A$ (в частности, при $A=\mathbb{Z}$ ) вопрос о характеризации инвариантов С-кобордизма, допускающих локальные формулы, остается открытым даже в случае C = CS. Например, как доказано автором в [2], первое число Понтрягина (а также, никакое его кратное) не допускает целочисленной локальной формулы.

Рассмотрим еще два примера.

1. Кольцо $\Omega_{*}^{\mathrm{HS}}$ - кольцо кобордизмов ориентированных симплициальных гомологических многообразий. Хорошо известно, что для ориентированных симплициальных гомологических многообразий корректно определены рациональные классы Понтрягина и, в частности, числа Понтрягина (см., например, [23]). Как доказал Ч. Р. Ф. Маундер [32], числа Понтрягина являются инвариантами кобордизмов ориентированных гомологических многообразий. Следовательно, для них существуют рациональные локальные формулы. Таким образом, группа когомологий $H^{*}\left(\mathcal{T}_{\mathrm{HS}}^{*}(\mathbb{Q})\right)$ содержит подгруппу, аддитивно изоморфную кольцу полиномов от классов Понтрягина с рациональными коэффициентами. Н. Мартин [33] доказал, что $\Omega_{4}^{\mathrm{HS}} \cong \mathbb{Z} \oplus \Theta_{3}^{H}$, где $\Theta_{3}^{H}-$ группа $H$-кобордизмов (гомологических кобордизмов) трехмерных гомологических сфер. М. Фурута [34] доказал, что группа $\Theta_{3}^{H}$ содержит бесконечно порожденную свободную абелеву подгруппу. Таким образом, полиномы от классов Понтрягина далеко не исчерпывают группу $H^{*}\left(\mathcal{T}_{\mathrm{HS}}^{*}(\mathbb{Q})\right)$. Имеем

$$
H^{4}\left(\mathcal{T}_{\mathrm{HS}}^{*}(\mathbb{Q})\right) \cong \operatorname{Hom}\left(\Omega_{4}^{\mathrm{HS}}, \mathbb{Q}\right) \cong \mathbb{Q} \oplus \operatorname{Hom}\left(\Theta_{3}^{H}, \mathbb{Q}\right)
$$

Прямое слагаемое $\mathbb{Q}$ порождено первым числом Понтрягина. Локальные формулы для первого числа Понтрягина и локальные формулы для инвариантов кобордизмов, соответствующих элементам группы $\operatorname{Hom}\left(\Theta_{3}^{H}, \mathbb{Q}\right)$, имеют принципиально разную природу. Рассмотрим инвариант HS-кобордизмов $q$, соответствующий гомоморфизму $h: \Theta_{3}^{H} \rightarrow \mathbb{Q}$. Функция

$$
\mathcal{T}_{4}^{\mathrm{HS}} \stackrel{\iota}{\rightarrow} \Theta_{3}^{H} \stackrel{h}{\rightarrow} \mathbb{Q},
$$

где $\iota$ - отображение, сопоставляющее каждой симплициальной гомологической сфере ее класс $H$-кобордизмов, является локальной формулой для инварианта q. Отметим, что значение этой локальной формулы на трехмерной симплициальной гомологической сфере $Y$ зависит лишь от класса $H$-кобордизмов гомологической сферы $Y$ и не зависит от комбинаторики триангуляции $Y$. В частности, ее значение на любой комбинаторной сфере равно нулю. Если же $f$ - локальная формула для первого класса Понтрягина, значение $f(Y)$ с необходимостью существенно зависит от комбинаторики триангуляции $Y$. В частности, обязательно существует трехмерная комбинаторная сфера $Y$ такая, что $f(Y) \neq 0$.

ЗАмЕчАНИЕ 3.2. Локальные формулы для инвариантов кобордизмов, соответствующих элементам группы $\operatorname{Hom}\left(\Theta_{3}^{H}, A\right)$, где $A$ - абелева группа, тесно связаны со следующей конструкцией. В [11] Д. Сулливан сопоставил каждому 
ориентированному симплициальному гомологическому многообразию $Z$ симплициальный цикл

$$
\sum_{\substack{\sigma \text { симплекс } \\ \text { codim } \sigma=4}} \sigma \otimes[\operatorname{link} \sigma] \in C_{\operatorname{dim} Z-4}\left(Z ; \Theta_{3}^{H}\right),
$$

класс гомологий которого является полным препятствием к существованию разрешения особенностей $g: M \rightarrow Z$ такого, что $M$ - комбинаторное многообразие и множество $g^{-1}(z)$ ациклично для любой точки $z \in Z$.

2. В [19] Дж. Чигер построил вещественные локальные формулы для гомологических $L$-классов Хирцебруха псевдомногообразий с пренебрежимой границей. В частности, для каждого натурального $k$ он получил вещественную локальную формулу для сигнатуры $4 k$-мерного псевдомногообразия с пренебрежимой границей. Таким образом, для каждого натурального числа $n$, кратного $4, \mathbb{R}$-модуль $H^{n}\left(\mathcal{T}_{\mathrm{Ch}}^{*}(\mathbb{R})\right)$ содержит одномерный подмодуль, порожденный классом когомологий локальных формул для сигнатуры. На самом деле это же утверждение верно и над полем рациональных чисел, так как рациональные локальные формулы для сигнатуры получаются из вещественных при помощи произвольной $\mathbb{Q}$-линейной проекции $\mathbb{R} \rightarrow \mathbb{Q}$. $\mathrm{K}$ сожалению, никакого более естественного способа получения рациональных локальных формул для сигнатуры псевдомногообразий с пренебрежимой границей пока не известно.

3.5. Связь с кобордизмами Сулливана-Бааса. Пусть $\mathrm{C}=\mathrm{C}\left(P_{1}, P_{2}, \ldots\right)$. В этом случае кольцо кобордизмов $\Omega_{*}^{\mathrm{C}}$ можно рассматривать как кольцо кобордизмов с особенностями "типа джойна" $P_{i_{1}} * \cdots * P_{i_{k}}, i_{1}<i_{2}<\cdots<i_{k}$. Идея рассмотрения таких кобордизмов и их первые приложения принадлежат Д. Сулливану (см. [8], [9], [11]). Первое строгое построение теории кобордизмов с особенностями типа джойна было произведено Н. Баасом [10]. (Он работал в гладкой категории, но мы будем рассматривать кусочно линейный аналог его конструкции.) Группы кобордизмов, построенные Н. Баасом, отличны от групп $\Omega_{*}^{\mathrm{C}}$. Проиллюстрируем причины этого отличия на примере случая с одним типом особенности $P$.

Многообразие с особенностями типа $P$ в смысле Сулливана-Бааса - это полиэдр вида

$$
V \cup_{W \times P}(W \times \text { cone }(P)),
$$

где $W$ - кусочно линейное многообразие без края и $V$ - кусочно линейное многообразие с краем $W \times P$. Оказывается, что определенное нами выше понятие многообразия с особенностями из класса $\mathrm{C}(P)$ шире, чем понятие многообразия с особенностями типа $P$ в смысле Сулливана-Бааса. Действительно, пусть $E \rightarrow B-$ произвольное кусочно линейное расслоение со слоем $P$ и замкнутым кусочно линейным многообразием в качестве базы, и пусть $V$ - кусочно линейное многообразие с краем $E$. Обозначим через $Z \rightarrow B$ ассоциированное с $E$ расслоение со слоем соne $(P)$. Рассмотрим полиэдр $X=V \cup_{E} Z$. Несложно проверить, что согласно нашему определению из п. 3.2 полиэдр $X$ является многообразием с особенностями из класса $\mathrm{C}(P)$. Однако $X$ может не быть многообразием с особенностями типа $P$ в смысле Сулливана-Бааса, если расслоение $E$ нетривиально. 


\section{§4. Разрешение особенностей и реализация циклов}

В этом и следующем параграфах мы не будем использовать индекс CS в обозначениях групп $\mathcal{T}_{*}^{\mathrm{CS}}$ и $\mathcal{T}_{\mathrm{CS}}^{*}(A)$.

4.1. Простые клетки. Пусть $K$ - комбинаторное многообразие. Для каждой вершины $y$ комплекса $K$ определим двойственную ей клетку равенством $D y=\operatorname{star}_{K^{\prime}} y$. Таким образом, $D y$ - объединение всех замкнутых симплексов комплекса $K^{\prime}$, содержащих вершину $y$. Клетку $D \sigma$, двойственную симплексу $\sigma$ комбинаторного многообразия $K$, определим как пересечение клеток, двойственных вершинам симплекса $\sigma$. Если комбинаторное многообразие $K$ и симплекс $\sigma$ ориентированы, клетка $D \sigma$ будет наделяться такой ориентацией, что произведение ориентации симплекса $\sigma$ на ориентацию клетки $D \sigma$ дает заданную ориентацию многообразия $K$. Клеточное разбиение, состоящее из клеток $D \sigma$, мы будем обозначать через $K^{*}$ и называть клеточным разбиением, двойственным триангуляиии $K$. Определение клеточного разбиения, двойственного кубически клеточному комбинаторному многообразию, дословно повторяет определение клеточного разбиения, двойственного симплициальному комбинаторному многообразию, если везде рассматривать кубы вместо симплексов.

Пусть теперь $Y$ - комбинаторная сфера. Рассмотрим симплициальный комплекс cone $\left(Y^{\prime}\right)$, кусочно линейно гомеоморфный стандартному диску. Разбиение $Y^{*}$ границы этого симплициального комплекса задает на нем структуру многообразия с углами. Дополним разбиение $Y^{*}$ до клеточного разбиения всего симплициального комплекса cone $\left(Y^{\prime}\right)$, положив $D \varnothing=\operatorname{cone}\left(Y^{\prime}\right)$. Симплициальный комплекс cone $\left(Y^{\prime}\right)$ с таким разбиением на клетки мы будем называть простой клеткой, двойственной комбинаторной сфере $Y$. Если комбинаторная сфера $Y$ реализована как граница выпуклого симплициального многогранника, двойственная ей простая клетка может быть реализована как двойственный простой многогранник.

Клетка $D \sigma$, двойственная симплексу $\sigma$ комбинаторного многообразия $K$, имеет каноническую структуру $(\operatorname{dim} K-\operatorname{dim} \sigma)$-мерной простой клетки, двойственной комбинаторной сфере $\operatorname{link} \sigma$. (Аналогичное утверждение верно для кубически клеточных комбинаторных многообразий.) В частности, каждая грань простой клетки имеет каноническую структуру простой клетки.

Работая с простыми клетками, нам будет удобно всегда помнить о триангуляции cone $\left(Y^{\prime}\right)$. Это означает, что простая клетка всегда рассматривается как полиэдр с двумя клеточными разбиениями: более крупным - разбиением на грани и его измельчением - триангуляцией cone $\left(Y^{\prime}\right)$. В частности, изоморфизмом простых клеток $P_{1}$ и $P_{2}$, двойственных комбинаторным сферам $Y_{1}$ и $Y_{2}$ соответственно, мы будем называть симплициальный изоморфизм $\operatorname{cone}\left(Y_{1}^{\prime}\right) \rightarrow \operatorname{cone}\left(Y_{2}^{\prime}\right)$, сохраняющий разбиение на грани.

(Конечным) комплексом из простых клеток называется факторпространство несвязного объединения конечного количества простых клеток $P_{1}, P_{2}, \ldots, P_{q}$ по отношению эквивалентности $\sim$ такому, что:

$1)$ отношение эквивалентности $\sim$ не отождествляет никакие две различные точки одной простой клетки $P_{i}$; 
2) если отношение эквивалентности $\sim$ отождествляет точку $x_{1} \in P_{i}$ с точкой $x_{2} \in P_{j}$, то оно отождествляет некоторую грань $F_{1} \subset P_{i}$, содержащую точку $x_{1}$, с некоторой гранью $F_{2} \subset P_{j}$, содержащей точку $x_{2}$, вдоль некоторого изоморфизма.

Образы граней простых клеток $P_{i}$ при такой факторизации называются клетками этого комплекса. Если все простые клетки $P_{i}$ являются симплексами или кубами, мы приходим к определению симплициально клеточного или кубически клеточного комплекса соответственно. Основным примером комплекса из простых клеток является разбиение $K^{*}$, двойственное комбинаторному многообразию $K$.

Барицентрическим подразделением простой клетки $P$, двойственной комбинаторной сфере $Y$, называется симплициальный комплекс $\operatorname{cone}\left(Y^{\prime}\right)$. Пусть $Z-$ комплекс из простых клеток. Подразделив барицентрически все клетки этого комплекса, мы получим симплициальный комплекс, который мы будем обозначать через $Z^{\prime}$ и называть барицентрическим подразделением комплекса $Z$. В частности, $\left(K^{*}\right)^{\prime}=K^{\prime}$.

Комплекс из простых клеток называется $n$-мерным псевдомногообразием, разбитым на простые клетки, если каждая его клетка содержится в некоторой $n$-мерной клетке, причем каждая $(n-1)$-мерная клетка содержится ровно в двух $n$-мерных клетках. Так же, как для симплициально клеточных и кубически клеточных псевдомногообразий, псевдомногообразие, разбитое на простые клетки, называется нормальным, если его старшая локальная группа гомологий в каждой точке изоморфна группе целых чисел. В дальнейшем все псевдомногообразия предполагаются нормальными.

4.2. Кобордизмы простых клеток. Границей $\partial P$ ориентированной простой клетки $P$ мы будем называть формальную сумму ее гиперграней, взятых с индуцированными ориентациями. Будем говорить, что сумма ориентированных $n$-мерных простых клеток $P_{1}+P_{2}+\cdots+P_{k}$ кобордантна сумме ориентированных $n$-мерных простых клеток $Q_{1}+Q_{2}+\cdots+Q_{l}$, если существуют ориентированные $n$-мерные простые клетки $R_{1}, R_{2}, \ldots, R_{m}$ и ориентированные $(n+1)$-мерные простые клетки $T_{1}, T_{2}, \ldots, T_{q}$ такие, что имеет место изоморфизм

$$
\begin{aligned}
\partial\left(T_{1}\right. & \left.+T_{2}+\cdots+T_{q}\right) \cong P_{1}+P_{2}+\cdots+P_{k}+R_{1}+R_{2}+\cdots+R_{m} \\
& +\left(-Q_{1}\right)+\left(-Q_{2}\right)+\cdots+\left(-Q_{l}\right)+\left(-R_{1}\right)+\left(-R_{2}\right)+\cdots+\left(-R_{m}\right) .
\end{aligned}
$$

Соответствующую группу кобордизмов мы будем называть группой кобордизмов $n$-мерных ориентированных простых клеток и обозначать через $\mathcal{P}_{n}$. Градуированная группа $\mathcal{P}_{*}$ является градуированным кольцом относительно операции прямого произведения простых клеток. Операция взятия границы простой клетки двойственна операции взятия суммы линков вершин комбинаторной сферы. Прямое произведение простых клеток двойственно джойну комбинаторных сфер. Поэтому кольцо $\mathcal{P}_{*}$ канонически изоморфно кольцу $H_{*}\left(\mathcal{T}_{*}\right)$. Таким образом, результаты из пп. $3.2,3.3$ могут быть переформулированы следующим образом.

Теорема 4.1. Функтор, ставящий в соответствие каждому ориентированному $n$-мерному комбинаторному многообразию $K$ формальную сумму 
$n$-мерных клеток двойственного разбиения $K^{*}$, задает корректно определеннъй аддитивный гомоморфизм $\psi: \Omega_{n}^{\mathrm{SPL}} \rightarrow \mathcal{P}_{n}$. Гомоморфизм $\psi$ является мультипликативным с точностью до элементов порядка 2. Ядро и коядро гомоморфизма $\psi$ являются группами кручения. Таким образом, имеет место мультипликативный изоморфизм

$$
\mathcal{P}_{*} \otimes \mathbb{Q} \cong \Omega_{*}^{\mathrm{SPL}} \otimes \mathbb{Q} \cong \Omega_{*}^{\mathrm{SO}} \otimes \mathbb{Q} .
$$

ЗАмечАниЕ 4.1. Пусть $X$ - топологическое пространство. Рассматривая сингулярные простые клетки пространства $X$, т. е. непрерывные отображения $P \rightarrow X$, можно стандартным образом определить экстраординарную теорию гомологий $\mathcal{P}_{*}(X)$. При этом имеется естественный гомоморфизм $\psi: \Omega_{*}^{\mathrm{SPL}}(X) \rightarrow$ $\mathcal{P}_{*}(X)$, индуцирующий изоморфизм

$$
\mathcal{P}_{*}(X) \otimes \mathbb{Q} \cong \Omega_{*}^{\mathrm{SPL}}(X) \otimes \mathbb{Q} \cong H_{*}\left(X ; \Omega_{*}^{\mathrm{SPL}} \otimes \mathbb{Q}\right) \cong H_{*}\left(X ; \Omega_{*}^{\mathrm{SO}} \otimes \mathbb{Q}\right) .
$$

4.3. Разрешение особенностей псевдомногообразия. В этом пункте мы для каждого ориентированного $n$-мерного псевдомногообразия $Z$, разбитого на простые клетки, явно построим кубически клеточное комбинаторное многообразие $M$ и кусочно гладкое отображение $g: M \rightarrow Z$ такие, что:

$1)$ ограничение отображения $g$ на множество $g^{-1}(Z \backslash \Sigma)$ есть конечнолистное накрытие

$$
g^{-1}(Z \backslash \Sigma) \rightarrow Z \backslash \Sigma,
$$

где $\Sigma$ - остов коразмерности 2 разбиения $Z$;

2) $\operatorname{dim} g^{-1}(\Sigma)=n-1$.

Пусть $P_{1}, P_{2}, \ldots, P_{k}$ - все $n$-мерные клетки псевдомногообразия $Z$. Рассмотрим ориентированные $(n-1)$-мерные комбинаторные сферы $Y_{1}, Y_{2}, \ldots, Y_{k}$ такие, что $P_{i}=\operatorname{cone}\left(Y_{i}^{\prime}\right)$ - простая клетка, двойственная комбинаторной сфере $Y_{i}$. Обозначим через $f_{i}$ вложение

$$
\operatorname{cone}\left(Y_{i}^{\prime}\right)=P_{i} \subset Z
$$

Для каждого симплекса $\sigma$ комплекса $Y_{i}$ положим $z(\sigma)=f_{i}(b(\sigma))$, где $b(\sigma)-$ барицентр симплекса $\sigma$. Положим

$$
Y=Y_{1} \sqcup Y_{2} \sqcup \cdots \sqcup Y_{k} .
$$

Так же, как в $\S 2$, мы будем обозначать через $U$ множество $(n-1)$-мерных симплексов комбинаторного многообразия $Y^{\prime}$. Симплексы $u \in U$ находятся во взаимно однозначном соответствии с последовательностями $\sigma^{0} \subset \sigma^{1} \subset \cdots \subset \sigma^{n-1}$ симплексов комплекса $Y$. Пусть $u$ - симплекс комбинаторной сферы $Y_{i}^{\prime}$, соответствующий последовательности $\sigma^{0} \subset \sigma^{1} \subset \cdots \subset \sigma^{n-1}$. Введем обозначения

$$
b_{j}(u)=b\left(\sigma^{j-1}\right), \quad z_{j}(u)=z\left(\sigma^{j-1}\right)=f_{i}\left(b_{j}(u)\right), \quad j=1,2, \ldots, n .
$$

Тогда $b_{1}(u), b_{2}(u), \ldots, b_{n}(u)$ - вершины симплекса $u$. Обозначим через $z_{0}(u)$ барицентр простой клетки $P_{i} \subset Z$. Несложно проверить, что каждая точка $z_{j}(u)$ является барицентром $(n-j)$-мерной клетки $F_{j}(u)$ разбиения $Z$, причем

$$
F_{n}(u) \subset F_{n-1}(u) \subset \cdots \subset F_{0}(u)=P_{i} .
$$


Рассмотрим процесс склейки псевдомногообразия $Z$ из простых клеток $P_{i}$. Он имеет следующий вид: гиперграни клеток $P_{1}, P_{2}, \ldots, P_{k}$ разбиваются на пары и в каждой паре склеиваются вдоль некоторого антиизоморфизма. Переходя к двойственным объектам, мы получаем инволюцию $\lambda: y \mapsto \tilde{y}$ на множестве $\operatorname{Vert}(Y)$ и набор антиизоморфизмов $\chi_{y}: \operatorname{star} y \rightarrow \operatorname{star} \tilde{y}$ таких, что $\chi_{\tilde{y}}=\chi_{y}^{-1}$. Применим к комбинаторному многообразию $Y$ конструкцию из п. 2.5. Эта конструкция дает нам однородный граф $Г$ степени $2 n$ такой, что $X=\widetilde{\mathbf{Q}}(\Gamma)-$ кубически клеточное комбинаторное многообразие, удовлетворяющее требованиям теоремы 2.3. (Псевдомногообразие $X$ является комбинаторным многообразием, так как линки всех его вершин являются комбинаторными сферами.) Сейчас нам будет удобно не переходить к большим кубам и работать с кубически клеточным комбинаторным многообразием $M=\mathbf{Q}(\Gamma)$. Напомним, что многообразие $M$ имеет вид

$$
M=\left(U \times \mathcal{B} \times S \times[0,1]^{n}\right) / \sim,
$$

где отношение эквивалентности $\sim$ порождено отождествлениями

$$
\begin{aligned}
& (u, \nu, s, \mathbf{t}) \sim\left(\Phi_{j}^{0}(u, \nu, s), \mathbf{t}\right), \quad \text { если } \quad t_{j}=0, \\
& (u, \nu, s, \mathbf{t}) \sim\left(\Phi_{j}^{1}(u, \nu, s), \mathbf{t}\right), \quad \text { если } \quad t_{j}=1 .
\end{aligned}
$$

Здесь $\mathbf{t}=\left(t_{1}, t_{2}, \ldots, t_{n}\right)$ - точка куба $[0,1]^{n}$.

Конечные множества $\mathcal{B}$ и $S$ и инволюции

$$
\Phi_{j}^{e}: U \times \mathcal{B} \times S \rightarrow U \times \mathcal{B} \times S
$$

были описаны в п. 2.5. Инволюции $\Phi_{j}^{0}$ имеют вид

$$
\Phi_{j}^{0}(u, \nu, s)=\left(\Phi_{j}(u), \nu, s\right) .
$$

Непосредственно из определения инволюций $\Phi_{j}$ следует, что $b_{l}\left(\Phi_{j}(u)\right)=b_{l}(u)$ при $l \neq j$. Кроме того, симплексы $u$ и $\Phi_{j}(u)$ лежат в одной компоненте связности комбинаторного многообразия $Y^{\prime}$. Следовательно, $z_{l}\left(\Phi_{j}(u)\right)=z_{l}(u)$ при $0 \leqslant l \leqslant n, l \neq j$.

Нам понадобится следующее свойство инволюций $\Phi_{j}^{1}$. Его доказательство мы отложим до п. 4.4.

ПРЕДЛОЖЕНИЕ 4.1. Если $\Phi_{j}^{1}\left(u_{1}, \nu_{1}, s_{1}\right)=\left(u_{2}, \nu_{2}, s_{2}\right)$, mo $z_{l}\left(u_{1}\right)=z_{l}\left(u_{2}\right)$ nрu $l \geqslant j$.

Если $z_{0}, z_{1}, \ldots, z_{n}$ - вершины некоторого симплекса $\rho$ комплекса $Z^{\prime}$ и $\alpha_{0}, \alpha_{1}, \ldots, \alpha_{n}$ - неотрицательные вещественные числа такие, что $\alpha_{0}+\alpha_{1}+\cdots$ $\cdots+\alpha_{n}=1$, мы будем использовать обозначение $\alpha_{0} z_{0}+\alpha_{1} z_{1}+\cdots+\alpha_{n} z_{n}$ для точки симплекса $\rho$ с барицентрическими координатами $\alpha_{0}, \alpha_{1}, \ldots, \alpha_{n}$.

Рассмотрим функции

$$
\begin{aligned}
\alpha_{0}(\mathbf{t}) & =\left(1-t_{1}\right)\left(1-t_{2}\right)\left(1-t_{3}\right) \ldots\left(1-t_{n}\right), \\
\alpha_{1}(\mathbf{t}) & =t_{1}\left(1-t_{2}\right)\left(1-t_{3}\right) \ldots\left(1-t_{n}\right), \\
\alpha_{2}(\mathbf{t}) & =t_{2}\left(1-t_{3}\right) \ldots\left(1-t_{n}\right), \\
\ldots \ldots \ldots \ldots \ldots \ldots \ldots & \ldots \ldots \ldots \\
\alpha_{n-1}(\mathbf{t}) & =t_{n-1}\left(1-t_{n}\right), \\
\alpha_{n}(\mathbf{t}) & =t_{n} .
\end{aligned}
$$


Очевидно, что

$$
\alpha_{0}(\mathbf{t})+\alpha_{1}(\mathbf{t})+\cdots+\alpha_{n}(\mathbf{t})=1 .
$$

Определим отображение $\underline{g}: U \times \mathcal{B} \times S \times[0,1]^{n} \rightarrow Z$ по формуле

$$
\underline{g}(u, \nu, s, \mathbf{t})=\alpha_{0}(\mathbf{t}) z_{0}(u)+\alpha_{1}(\mathbf{t}) z_{1}(u)+\cdots+\alpha_{n}(\mathbf{t}) z_{n}(u) .
$$

ПРЕДЛОЖЕНИЕ 4.2. Имеют место равенства

$$
\begin{array}{lll}
\underline{g}(u, \nu, s, \mathbf{t})=\underline{g}\left(\Phi_{j}^{0}(u, \nu, s), \mathbf{t}\right), & \text { если } \quad t_{j}=0, \\
\underline{g}(u, \nu, s, \mathbf{t})=\underline{g}\left(\Phi_{j}^{1}(u, \nu, s), \mathbf{t}\right), \quad \text { еслu } \quad t_{j}=1 .
\end{array}
$$

Таким образом, отображение g индуцирует корректно определенное отображение $g: M \rightarrow Z$.

ДокАЗАТЕЛЬство. Первое равенство следует из того, что $z_{l}\left(\Phi_{j}(u)\right)=z_{l}(u)$ при $l \neq j$ и $\alpha_{j}(\mathbf{t})=0$, если $t_{j}=0$. Второе равенство следует из предложения 4.1, потому что $\alpha_{l}(\mathbf{t})=0$, если $t_{j}=1$ и $l<j$.

Обозначим через $H$ объединение гиперграней $\left\{t_{j}=1\right\}, j=2,3, \ldots, n$, куба $[0,1]^{n}$, через $\Xi \subset M-$ образ множества $U \times \mathcal{B} \times S \times H$ при отображении факторизации

$$
U \times \mathcal{B} \times S \times[0,1]^{n} \rightarrow M .
$$

Очевидно, что $\Xi=g^{-1}(\Sigma)$ и $\operatorname{dim} \Xi=n-1$. То, что отображение

$$
\left.g\right|_{M \backslash \Xi: M \backslash \Xi \rightarrow Z \backslash \Sigma}
$$

является накрытием, легко следует из того, что система уравнений

$$
\alpha_{j}(\mathbf{t})=\beta_{j}, \quad j=0,1, \ldots, n,
$$

где $\beta_{j}$ - неотрицательные вещественные числа такие, что $\beta_{0}+\beta_{1}+\cdots+\beta_{n}=1$, однозначно разрешима, если $\beta_{0} \neq 0$ или $\beta_{1} \neq 0$.

ЗАмечАниЕ 4.2. Комплекс $M$ является каноническим подразделением кубически клеточного комбинаторного многообразия $X$ (см. п. 2.4). Рассмотрим двойственное разбиение $X^{*}$. Клетка $Q$ разбиения $X^{*}$, двойственная вершине $x$ комплекса $X$, есть объединение всех замкнутых кубов разбиения $M$, содержащих вершину $x$. Если $\operatorname{link} x \cong Y_{i}^{\prime}$, то $Q$ - простая клетка, двойственная комбинаторной сфере $Y_{i}^{\prime}$. По построению $g$ отображает простую клетку $Q$ на простую клетку $P_{i}$. При этом $g$ взаимно однозначно на внутренности клетки $Q$ и на внутренностях гиперграней клетки $Q$, двойственных тем вершинам $y \in Y_{i}^{\prime}$, которые являются вершинами комплекса $Y_{i}$. Остальные гиперграни простой клетки $Q$ отображение $g$ "схлопывает" на грани клетки $P_{i}$ меньшей размерности. Пусть $F$ - грань клетки $Q$, двойственная симплексу $\tau$ комбинаторной сферы $Y_{i}^{\prime}$. Возможны два варианта.

1. Симплекс $\tau$ не содержится ни в каком таком симплексе $\sigma$ комплекса $Y_{i}$, что $\operatorname{dim} \sigma=\operatorname{dim} \tau$. Тогда линк симплекса $\tau$ обладает антиавтоморфизмом. Значит, грань $F$ обладает антиавтоморфизмом. Несложно проверить, что каждую такую грань $F$ отображение $g$ переводит в грань меньшей размерности. 
2. Симплекс $\tau$ содержится в некотором симплексе $\sigma$ комплекса $Y_{i}$ таком, что $\operatorname{dim} \sigma=\operatorname{dim} \tau$. Пусть $E-$ грань клетки $P_{i}$, двойственная симплексу $\sigma$. Тогда $g$ отображает клетку $F$ на клетку $E$, так что внутренность клетки $F$ отображается гомеоморфно на внутренность клетки $E$. Отметим, что комбинаторная сфера $\operatorname{link}_{Y_{i}^{\prime}} \tau$, двойственная клетке $F$, изоморфна барицентрическому подразделению комбинаторной сферы $\operatorname{link}_{Y_{i}} \sigma$, двойственной клетке $E$.

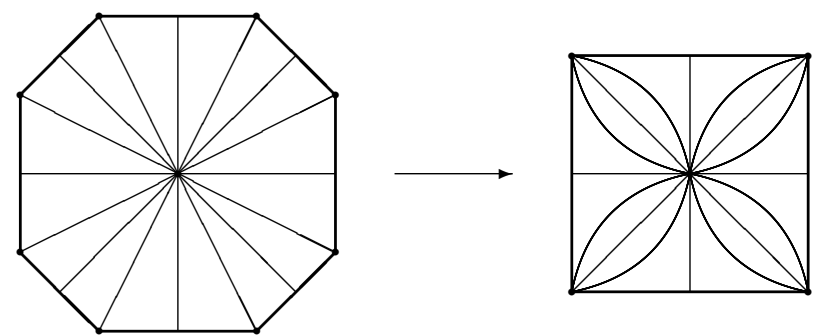

Рис. 2

Например, пусть $Y_{i}$ - граница четырехугольника. Тогда $P_{i}$ - четырехугольник, $Q$ - восьмиугольник. Отображение $\left.g\right|_{Q}$ изображено на рис. 2. Наклонные стороны восьмиугольника $Q$ отображаются на вершины четырехугольника $P_{i}$.
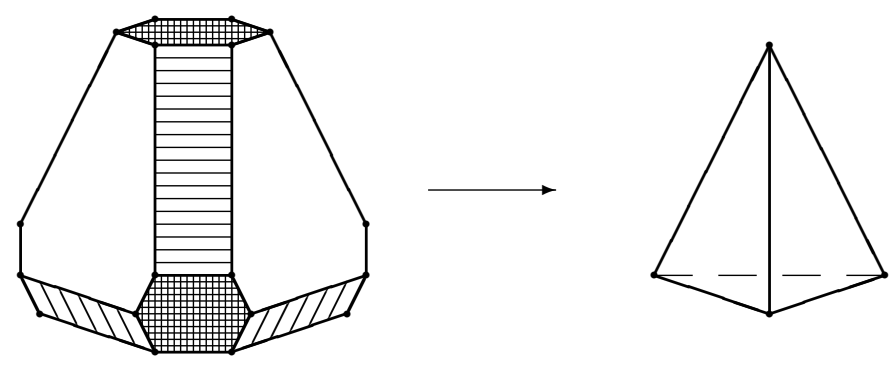

Рис. 3

Другой пример изображен на рис. 3. Здесь $Y_{i}$ - граница тетраэдра. Тогда $P_{i}$ - тетраэдр, $Q$ - многогранник, изображенный на рис. 3 слева. Заштрихованные шестиугольные грани многогранника $Q$ отображаются на соответствующие вершины тетраэдра. Четырехугольные грани многогранника $Q$ отображаются на ребра тетраэдра, причем каждый отрезок, параллельный направлению штриховки, "схлопывается" в точку. Внутренности незаштрихованных шестиугольных граней многогранника $Q$ отображаются гомеоморфно на внутренности соответствующих граней тетраэдра.

4.4. Доказательство предложения 4.1. Мы будем пользоваться всеми обозначениями из п. 2.5. Единственным отличием будет то, что сейчас мы рассматриваем множество $S$ и инволюции $\Phi_{j}^{e}$, построенные по комбинаторному 
многообразию $\bar{Y}=Y \times \mathcal{B}$, а не по $Y$. В частности, граф $G$ будет графом на множестве вершин $\bar{W}=W \times \mathcal{B}$, а не на множестве $W$. Мы будем использовать обозначение $\pi$ для проекции $W \times \mathcal{B} \rightarrow W$. Положим $\bar{z}=z \circ \pi$. Как было отмечено в п. 2.5, для двух симплексов $\rho_{1}$ и $\rho_{2}$ комплекса $\bar{Y}$ может существовать не более одного антиизоморфизма star $\rho_{1} \rightarrow \operatorname{star} \rho_{2}$, сохраняющего метки вершин. Если такой антиизоморфизм существует, мы будем обозначать его через $\omega_{\rho_{1}, \rho_{2}}$. В частности, если $\bar{y}=(y, \nu)-$ вершина комплекса $\bar{Y}$, то $\omega_{\bar{y}, \tilde{y}}=\bar{\chi}_{\bar{y}}-$ антиизоморфизм, индуцированный антиизоморфизмом $\chi_{y}$.

Из определения антиизоморфизмов $\chi_{y}$ сразу следует, что $z\left(\chi_{y}(\sigma)\right)=z(\sigma)$ для любого симплекса $\sigma \in W$, содержащего вершину $y$. Значит, $\bar{z}\left(\bar{\chi}_{\bar{y}}(\sigma)\right)=\bar{z}(\sigma)$ для любого симплекса $\sigma \in \bar{W}$, содержащего вершину $\bar{y}$. Пусть $\rho_{1}, \rho_{2} \in \bar{W}-$ два симплекса, соединенных ребром в графе $G$. Тогда существует вершина $\bar{y} \in \rho_{1}$ такая, что $\bar{\chi}_{\bar{y}}\left(\rho_{1}\right)=\rho_{2}$. Антиизоморфизм $\omega_{\rho_{1}, \rho_{2}}$ является ограничением антиизоморфизма $\bar{\chi}_{\bar{y}}$ на подкомплекс $\operatorname{star} \rho_{1} \subset \operatorname{star} \bar{y}$. Поэтому $\bar{z}\left(\omega_{\rho_{1}, \rho_{2}}(\sigma)\right)=\bar{z}(\sigma)$ для любого симплекса $\sigma \supset \rho_{1}$. Следовательно, это же равенство $\bar{z}\left(\omega_{\rho_{1}, \rho_{2}}(\sigma)\right)=\bar{z}(\sigma)$ имеет место для любых симплексов $\rho_{1}, \rho_{2}$, лежащих в разных долях одной компоненты связности графа $G$, и любого симплекса $\sigma \supset \rho_{1}$. Значит, $\bar{z}\left(\Lambda_{c}(\sigma)\right)=\bar{z}(\sigma)$ для любых $\Lambda \in P, c \subset \mathcal{C}$ таких, что $c(\sigma) \supset c$.

Пусть $\sigma_{i}^{0} \subset \sigma_{i}^{1} \subset \cdots \subset \sigma_{i}^{n-1}, i=1,2,-$ последовательности симплексов комплекса $\bar{Y}$, соответствующие симплексам $\left(u_{i}, \nu_{i}\right)$ комплекса $\bar{Y}^{\prime}$. Положим $c=c\left(\sigma_{1}^{j-1}\right)$. Тогда $\sigma_{2}^{l-1}=\Lambda_{c}\left(\sigma_{1}^{l-1}\right), l=1,2, \ldots, n$. Следовательно, при $l \geqslant j$ получаем $\bar{z}\left(\sigma_{2}^{l-1}\right)=\bar{z}\left(\sigma_{1}^{l-1}\right)$, что доказывает предложение 4.1.

4.5. Класс кобордизмов многообразия $M$. Разрешение особенностей псевдомногообразия может приводить к многообразиям, представляющим разные классы кобордизмов. Мы занимаемся разрешением особенностей с кратностями, т. е. рассматриваем отображения $M \rightarrow Z$, которые на открытом всюду плотном подмножестве являются конечнолистными накрытиями. Поэтому в нашем случае имеет смысл рассматривать класс $\frac{[M]}{r} \in \Omega_{n}^{\mathrm{SPL}} \otimes \mathbb{Q}=\Omega_{n}^{\mathrm{SO}} \otimes \mathbb{Q}$, где $r$ - число листов накрытия. Оказывается, что наша конструкция из п. 4.3 дает многообразие $[M]$ с вполне определенным классом $\frac{[M]}{r}$, полностью определяемым набором простых клеток $P_{1}, P_{2}, \ldots, P_{k}$.

Теорема 4.2. Пусть $Z$ - ориентированное $n$-мерное псевдомногообразие, разбитое на простые клетки, $\left(P_{1}, P_{2}, \ldots, P_{k}\right)$ - набор его $n$-мерных клеток. Пусть $M$ - многообразие, построенное в $n .4 .3, r$ - число листов накрытия $M \backslash \Xi \rightarrow Z \backslash \Sigma$. Тогда

$$
\frac{[M]}{r}=\psi^{-1}\left(\left[P_{1}+P_{2}+\cdots+P_{k}\right]\right),
$$

где $\psi: \Omega_{n}^{\mathrm{SPL}} \otimes \mathbb{Q} \rightarrow \mathcal{P}_{*} \otimes \mathbb{Q}-$ изоморбизм из теоремь 4.1 .

ДоказАтельство. Рассмотрим комбинаторное многообразие $M^{\prime}$. Набор линков его вершин состоит из набора $\left(Y_{1}^{\prime \prime}, Y_{2}^{\prime \prime}, \ldots, Y_{k}^{\prime \prime}\right)$, взятого $r$ раз, и некоторого набора комбинаторных сфер, каждая из которых обладает антиавтоморфизмом. Значит,

$$
2 d\left(M^{\prime}\right)=2 r \sum_{i=1}^{k} Y_{i}^{\prime \prime}
$$


в группе $\mathcal{T}_{n}$. Из предложения 2.13 следует, что циклы $2 \sum_{i=1}^{k} Y_{i}^{\prime \prime}$ и $2 \sum_{i=1}^{k} Y_{i}$ представляют одинаковые классы гомологий в группе $H_{n}\left(\mathcal{T}_{*}\right)$. Гомоморфизм $\psi$ соответствует гомоморфизму $d_{*}$ при каноническом изоморфизме $\mathcal{P}_{n} \cong H_{n}\left(\mathcal{T}_{*}\right)$. Следовательно,

$$
2 \psi([M])=2 r\left[P_{1}+P_{2}+\cdots+P_{k}\right]
$$

в группе $\mathcal{P}_{n}$.

4.6. Реализация симплициальных циклов. Пусть $R$ - топологическое пространство, $C_{*}^{\text {sing }}(R)$ - его сингулярный симплициальный цепной комплекс.

Пусть $\xi \in C_{n}^{\text {sing }}(R)$ - цикл. Цикл $\xi$ легко может быть реализован как образ фундаментального класса ориентированного симплициального псевдомногообразия $Z$ при некотором непрерывном отображении $h: Z \rightarrow R$. Симплекс можно рассматривать как простую клетку, двойственную границе симплекса. Таким образом, симплициальное псевдомногообразие есть частный случай псевдомногообразия, разбитого на простые клетки. Разрешим особенности псевдомногообразия $Z$ при помощи конструкции, описанной в п. 4.3. Образ фундаментального класса многообразия $M$ при сквозном отображении

$$
\varphi: M \stackrel{g}{\longrightarrow} Z \stackrel{h}{\longrightarrow} R
$$

равен классу $r[\xi]$, где $r$ - число листов накрытия $M \backslash \Xi \rightarrow Z \backslash \Sigma$.

Из того что все комбинаторные сферы $Y_{i}$, двойственные $n$-мерным клеткам псевдомногообразия $Z$, изоморфны границе симплекса, следует, что при $j<n$ линк каждого $j$-мерного симплекса комбинаторного многообразия $M^{\prime}$ обладает антиавтоморфизмом. Согласно результату Н. Левитта и К. Рурка [1] это свойство обеспечивает тривиальность всех рациональных классов Понтрягина многообразия $M$. Рассмотрим числа Понтрягина отображения $\varphi$ (см. [35]). Пусть $\omega=\left(i_{1}, i_{2}, \ldots, i_{m}\right),|\omega|=\sum i_{j}, b \in H^{n-|\omega|}(R)$. Тогда

$$
\left\langle p_{\omega}(M) \smile \varphi^{*} b,[M]\right\rangle=\left\{\begin{array}{lll}
0, & \text { если } & |\omega|>0, \\
r\langle b,[\xi]\rangle, & \text { если } & |\omega|=0 .
\end{array}\right.
$$

В [35] доказано, что класс $[\varphi] \in \Omega_{n}^{\mathrm{SPL}}(R) \otimes \mathbb{Q} \cong \Omega_{n}^{\mathrm{SO}}(R) \otimes \mathbb{Q}$, представленный отображением $\varphi$, полностью характеризуется своими числами Понтрягина. Следовательно, элемент $\frac{[\varphi]}{r} \in \Omega_{n}^{\mathrm{SPL}}(R) \otimes \mathbb{Q}$ не зависит от произвольного построения многообразия $M$ и не меняется при замене цикла $\xi$ на гомологичный. Таким образом, наша конструкция задает для каждого топологического пространства $R$ отображение

$$
\theta_{R}: H_{*}(R ; \mathbb{Z}) \rightarrow \Omega_{*}^{\mathrm{SPL}}(R) \otimes \mathbb{Q},
$$

являющееся правым обратным к отображению аугментации $\Omega_{*}^{\mathrm{SPL}}(R) \otimes \mathbb{Q} \rightarrow$ $H_{*}(R ; \mathbb{Q})$. Очевидно, что $\theta_{R}$ является естественным преобразованием теорий гомологий $H_{*}(\cdot ; \mathbb{Z}) \rightarrow \Omega_{*}^{\mathrm{SPL}}(\cdot) \otimes \mathbb{Q}$, совпадающим для одноточечного пространства со стандартным вложением $\mathbb{Z} \subset \Omega_{0}^{\mathrm{SPL}} \otimes \mathbb{Q}$. Следовательно, отображение $\theta_{R}$ совпадает с композицией

$$
H_{*}(R ; \mathbb{Z}) \stackrel{\eta}{\longrightarrow} H_{*}\left(R ; \Omega_{*}^{\mathrm{SPL}} \otimes \mathbb{Q}\right) \stackrel{\left(\mathrm{ch}^{\mathrm{SPL}}\right)^{-1}}{\longrightarrow} \Omega_{*}^{\mathrm{SPL}}(R) \otimes \mathbb{Q},
$$


где $\eta$ - гомоморфизм, индуцированный стандартным вложением $\mathbb{Z} \subset \Omega_{0}^{\mathrm{SPL}} \otimes \mathbb{Q}$,

$$
\operatorname{ch}^{\mathrm{SPL}}: \Omega_{*}^{\mathrm{SPL}}(R) \rightarrow H_{*}\left(R ; \Omega_{*}^{\mathrm{SPL}} \otimes \mathbb{Q}\right)
$$

- характер Чженя-Дольда в теории ориентированных кусочно линейных бордизмов.

4.7. Реализация циклов, двойственных симплициальным коциклам. Пусть $R$ - ориентированное $m$-мерное комбинаторное многообразие, $C^{*}(R ; \mathbb{Z})$ - комплекс его симплициальных коцепей. Пусть $c \in C^{m-n}(R ; \mathbb{Z})-$ коцикл. Двойственный ему цикл $\xi$ лежит в группе $C_{n}\left(R^{*} ; \mathbb{Z}\right)$ клеточных цепей разбиения $R^{*}$. Предположим, что мы хотим реализовать класс гомологий, кратный классу $[\xi]$, как образ фундаментального класса многообразия. Конечно, мы легко можем заменить цикл $\xi$ на гомологичный ему симплициальный цикл в группе $C_{n}\left(R^{\prime} ; \mathbb{Z}\right)$ и таким образом свести задачу к ситуации, рассмотренной в п. 4.6. Более интересные результаты получаются, если рассматривать цикл $\xi$ как цикл, состоящий из простых клеток разбиения $R^{*}$.

Итак, цикл $\xi$ легко может быть представлен как образ фундаментального цикла псевдомногообразия $Z$, разбитого на простые клетки, при отображении $h$, которое отображает каждую клетку разбиения $Z$ изоморфно на некоторую клетку разбиения $R^{*}$. Разрешим особенности псевдомногообразия $Z$ при помощи конструкции, описанной в п. 4.3. Образ фундаментального класса многообразия $M$ при сквозном отображении

$$
\varphi: M \stackrel{g}{\longrightarrow} Z \stackrel{h}{\longrightarrow} R
$$

равен классу $r[\xi]$, где $r$ - число листов накрытия $M \backslash \Xi \rightarrow Z \backslash \Sigma$.

ПРЕДЛОЖЕНИЕ 4.3. Отображение $\varphi^{*}$ переводит рационалъные классы Понтрягина многообразия $R$ в рачиональные классы Понтрягина многообразия $M$.

Доказательство этого утверждения будет дано в следующем пункте.

Числа Понтрягина отображения $\varphi$ имеют вид

$$
\left\langle p_{\omega}(M) \smile \varphi^{*} b,[M]\right\rangle=r\left\langle p_{\omega}(R) \smile b,[\xi]\right\rangle .
$$

Значит, элемент $\frac{[\varphi]}{r} \in \Omega_{n}^{\mathrm{SPL}}(R) \otimes \mathbb{Q}$ не зависит от произвольного построения многообразия $M$ и не меняется при замене цикла $\xi$ на гомологичный. Таким образом, наша конструкция задает для каждого комбинаторного многообразия $R$ отображение

$$
\theta_{R^{*}}: H_{*}(R ; \mathbb{Z}) \rightarrow \Omega_{*}^{\mathrm{SPL}}(R) \otimes \mathbb{Q},
$$

являющееся правым обратным к отображению аугментации $\Omega_{*}^{\mathrm{SPL}}(R) \otimes \mathbb{Q} \rightarrow$ $H_{*}(R ; \mathbb{Q})$. В отличие от отображения $\theta_{R}$, отображение $\theta_{R^{*}}$ не является естественным преобразованием теорий гомологий. Оказывается, что отображение теорий когомологий, двойственное отображению $\theta_{R^{*}}$, является естественным преобразованием теорий когомологий. 
ПРЕДЛОЖЕНИЕ 4.4. Отображение $\theta_{R^{*}}$ совпадает с композицией

$$
\begin{aligned}
H_{*}(R ; \mathbb{Z}) \stackrel{D}{\rightarrow} H^{*}(R ; \mathbb{Z}) \stackrel{\eta}{\longrightarrow} H^{*}\left(R ; \Omega_{\mathrm{SPL}}^{*} \otimes \mathbb{Q}\right) \stackrel{\mathrm{ch}_{\mathrm{SPL}}^{-1}}{\longrightarrow} \\
\stackrel{\mathrm{ch}_{\mathrm{SPL}}^{-1}}{\longrightarrow} \Omega_{\mathrm{SPL}}^{*}(R) \otimes \mathbb{Q} \stackrel{D_{\mathrm{SPL}}^{-1} \otimes \mathbb{Q}}{\longrightarrow} \Omega_{*}^{\mathrm{SPL}}(R) \otimes \mathbb{Q},
\end{aligned}
$$

где $\eta$ - гомоморфизм, индуцированный вложением $\mathbb{Z} \subset \Omega_{\mathrm{SPL}}^{*} \otimes \mathbb{Q}$, a $D$ и $D_{\mathrm{SPL}}-$ операторы двойственности Пуанкаре в когомологиях и в ориентированных кусочно линейных кобордизмах соответственно и

$$
\operatorname{ch}_{\mathrm{SPL}}: \Omega_{\mathrm{SPL}}^{*}(R) \rightarrow H^{*}\left(R ; \Omega_{\mathrm{SPL}}^{*} \otimes \mathbb{Q}\right)
$$

- характер Чженя-Дольда в теории ориентированных кусочно линейных кобордизмов.

ДокАЗАТЕЛЬСтво. Обозначим через $\pi^{j}(R)=\lim _{\longrightarrow}\left[\Sigma^{q} R^{+}, S^{q+j}\right]$ стабильную когомотопическую группу пространства $R$. По теореме Ж.-П. Серра имеется естественный изоморфизм $H^{*}(R ; \mathbb{Q}) \cong \pi^{*}(R) \otimes \mathbb{Q}$. Согласно конструкции B. M. Бухштабера [36] отображение $\mathrm{ch}_{\mathrm{SPL}}^{-1}$, обратное характеру Чженя-Дольда в теории ориентированных кусочно линейных кобордизмов, представляется в виде композиции:

$$
H^{*}\left(R ; \Omega_{\mathrm{SPL}}^{*} \otimes \mathbb{Q}\right) \stackrel{\cong}{\longrightarrow} \pi^{*}(R) \otimes \Omega_{\mathrm{SPL}}^{*} \otimes \mathbb{Q} \stackrel{H \otimes \mathbb{Q}}{\longrightarrow} \Omega_{\mathrm{SPL}}^{*}(R) \otimes \mathbb{Q},
$$

где $H: \pi^{*}(R) \otimes \Omega_{\mathrm{SPL}}^{*} \rightarrow \Omega_{\mathrm{SPL}}^{*}(R)$ - гомоморфизм Гуревича, переводящий элемент $(a, \alpha), a \in \pi^{j}(R), \alpha \in \Omega_{\mathrm{SPL}}^{-l}$, в образ элемента $\alpha$ при гомоморфизме

$$
\Omega_{\mathrm{SPL}}^{-l} \cong \widetilde{\Omega}_{\mathrm{SPL}}^{q+j-l}\left(S^{q+j}\right) \stackrel{a^{*}}{\longrightarrow} \widetilde{\Omega}_{\mathrm{SPL}}^{q+j-l}\left(\Sigma^{q} R^{+}\right) \cong \Omega_{\mathrm{SPL}}^{j-l}(R) .
$$

Пусть $\alpha=1 \in \Omega_{\mathrm{SPL}}^{0}, R$ - ориентированное $m$-мерное комбинаторное многообразие. Положим $x=\left(D_{\mathrm{SPL}}^{-1} \circ H\right)(a, 1) \in \Omega_{m-j}^{\mathrm{SPL}}(R)$. Рассмотрим класс кобордизмов

$$
\gamma \in \widetilde{\Omega}_{\mathrm{SPL}}^{q+j}\left(\Sigma^{q} R^{+}\right)=\Omega_{\mathrm{SPL}}^{q+j}\left(R \times D^{q}, R \times S^{q-1}\right),
$$

являющийся образом фундаментального класса сферы $S^{q+j}$ при отображении $a^{*}$, и двойственный ему по Пуанкаре-Лефшецу класс бордизмов $y \in$ $\Omega_{m-j}^{\mathrm{SPL}}\left(R \times D^{q}\right)$. Очевидно, что класс $y$ переходит в класс $x$ при естественном изоморфизме $\Omega_{m-j}^{\mathrm{SPL}}\left(R \times D^{q}\right) \rightarrow \Omega_{m-j}^{\mathrm{SPL}}(R)$. Класс $y$ представляется подмногообразием с тривиальным нормальным расслоением - трансверсальным прообразом точки при отображении из гомотопического класса $a$. Таким образом, класс $x$ представляется отображением $\varkappa: N \rightarrow R$ таким, что $\varkappa^{*}\left(p_{i}(R)\right)=p_{i}(N)$, где $p_{i}$ - рациональные классы Понтрягина.

Пусть

$$
z=\left(\left(D_{\mathrm{SPL}}^{-1} \otimes \mathbb{Q}\right) \circ \operatorname{ch}_{\mathrm{SPL}}^{-1} \circ \eta \circ D\right)([\xi]) \in \Omega_{n}^{\mathrm{SPL}}(R) \otimes \mathbb{Q} .
$$

Тогда $z \in\left(D_{\mathrm{SPL}}^{-1} \circ H\right)\left(\pi^{*}(R)\right) \otimes \mathbb{Q}$. Значит, с некоторой кратностью класс $z$ представляется образом многообразия, рациональные классы Понтрягина которого индуцируются рациональными классами Понтрягина многообразия $R$. С другой стороны, по предложению 4.3 класс $\theta_{R^{*}}([\xi])$ также с некоторой кратностью 
представляется образом многообразия, рациональные классы Понтрягина которого индуцируются рациональными классами Понтрягина многообразия $R$. Равенство $\theta_{R^{*}}([\xi])=z$ следует из равенства чисел Понтрягина элементов $\theta_{R^{*}}([\xi])$ и $z$.

4.8. Доказательство предложения 4.3. В [2] автором было доказано следующее утверждение (см. также п. 5.1 настоящей статьи).

ПреДЛОЖЕНИЕ 4.5. Для каждого натуралъного l существует $\mathbb{Q}$-значная бункция $f$ на множестве классов изоморфизмов ориентированных $(4 l-1)$ мерных комбинаторных сфер такая, что $f(-Y)=-f(Y)$ и для любого комбинаторного многообразия $K$ цепь

$$
f_{\sharp}(K)=\sum_{\substack{\sigma-\text { симплекс } K \\ \text { codim } \sigma=4 l}} f(\operatorname{link} \sigma) \sigma
$$

является ииклом, класс гомологий которого двойствен по Пуанкаре l-му раииональному классу Понтрягина многообразия $K$.

СЛЕДСТВИЕ 4.1. Для любого комбинаторного многообразия $K$ и любого натурального числа $ј$ иепь

$$
f_{\sharp}^{(j)}(K)=\sum_{\substack{\sigma \text { - симплекс } \\ \text { содim } \sigma=4 l}} f\left((\operatorname{link} \sigma)^{(j)}\right) \sigma
$$

является циклом, класс гомологий которого двойствен по Пуанкаре классу $p_{l}(K)$. (Через $Y^{(j)}$ обозначено $j$-е барищентрическое подразделение комплекca $Y$.)

Это следствие можно вывести из предложения 2.13, но проще непосредственно заметить, что цепь $f_{\sharp}^{(j)}(K)$ является циклом, гомологичным циклу $f_{\sharp}\left(K^{(j)}\right)$. Точно так же получается следующее утверждение.

СЛЕДСТВИЕ 4.2. Для любого кубически клеточного комбинаторного многообразия $K$ и любого неотрицательного челого числа $j$ кубическая цепь

$$
f_{\sharp}^{(j)}(K)=\sum_{\substack{\sigma-\text { куб } K \\ \operatorname{codim} \sigma=4 l}} f\left((\operatorname{link} \sigma)^{(j)}\right) \sigma
$$

является ииклом, класс гомологий которого двойствен по Пуанкаре классу $p_{l}(K)$.

Пусть $Z$ - псевдомногообразие, разбитое на простые клетки. Определим клеточную коцепь $c^{(j)}(Z) \in C^{4 l}(Z ; \mathbb{Q})$, положив $c^{(j)}(Z)(P)=f\left(Y^{(j)}\right)$, если простая клетка $P$ двойственна комбинаторной сфере $Y$.

СлЕДСТВИЕ 4.3. Если $K$ - симплициалъно или кубически клеточное комбинаторное многообразие, то $\left[c^{(j)}\left(K^{*}\right)\right]=p_{l}(K)$.

Пусть $X$ - кубически клеточный комплекс, получаемый из $M$ после перехода к большим кубам (см. п. 2.4). Рассмотрим $4 l$-мерную клетку $Q$ комплекса $X^{*}$. Возможны два случая (см. замечание 4.2$)$ : 
1) $\operatorname{dim} g(Q)<4 l$;

2) $g$ отображает клетку $Q$ на некоторую $4 l$-мерную клетку $P$ разбиения $R^{*}$, причем внутренность клетки $Q$ отображается гомеоморфно на внутренность клетки $P$.

В случае 1$)$ клетка $Q$ обладает антиавтоморфизмом. Значит,

$$
c^{(0)}\left(X^{*}\right)(Q)=0
$$

В случае 2) если $P$ - простая клетка, двойственная комбинаторной сфере $Y$, то $Q$ - простая клетка, двойственная комбинаторной сфере $Y^{\prime}$. Значит,

$$
c^{(0)}\left(X^{*}\right)(Q)=c^{(1)}\left(R^{*}\right)(P) .
$$

Таким образом, $g^{*}\left(c^{(1)}\left(R^{*}\right)\right)=c^{(0)}\left(X^{*}\right)$. Следовательно, $g^{*}\left(p_{l}(R)\right)=p_{l}(M)$.

\section{§ 5. Локальные формулы для рациональных классов Понтрягина}

В этом параграфе мы рассматриваем комбинаторные сферы и комбинаторные многообразия и не используем индекс $\mathrm{CS}$ в обозначениях групп $\mathcal{T}_{*}^{\mathrm{CS}}$ и $\mathcal{T}_{\mathrm{CS}}^{*}(A)$. Отметим, однако, что все результаты из пп. 5.1-5.8 остаются в силе, если заменить класс CS на класс HS, т. е. рассматривать симплициальные гомологические сферы вместо комбинаторных сфер и симплициальные гомологические многообразия вместо комбинаторных многообразий.

Напомним, что согласно следствию 3.4 гомоморфизм

$$
\delta^{*}: H^{*}\left(\mathcal{T}^{*}(\mathbb{Q})\right) \rightarrow \operatorname{Hom}\left(\Omega_{*}^{\mathrm{SPL}}, \mathbb{Q}\right) \cong \mathbb{Q}\left[p_{1}, p_{2}, \ldots\right]
$$

является изоморфизмом.

Для простоты мы будем предполагать, что все рассматриваемые в данном параграфе комбинаторные многообразия являются ориентированными. Все результаты переносятся практически без изменений на случай неориентируемых комбинаторных многообразий, если вместо обычных симплициальных цепей использовать коориентированные симплициальные иепи (см. [16], а также [2]). Каждой функции $f \in \mathcal{T}^{n}(\mathbb{Q})$ и каждому $m$-мерному комбинаторному многообразию $K$ можно поставить в соответствие симплициальную цепь

$$
f_{\sharp}(K)=\sum_{\substack{\sigma-\text { симплекс } K \\ \text { codim } \sigma=n}} f(\operatorname{link} \sigma) \sigma .
$$

Отметим, что слагаемое $f(\operatorname{link} \sigma) \sigma$ не зависит от выбора ориентации симплекса $\sigma$. В [2] автором было доказано следующее утверждение.

ПРЕДЛОЖЕНИЕ 5.1. Цепъ $f_{\sharp}(K)$ является ииклом для любого комбинаторного многообразия $K$ тогда и толъко тогда, когда функиия $f$ является кочиклом комплекса $\mathcal{T}^{*}(\mathbb{Q})$. Класс гомологий цикла $f_{\sharp}(K)$ зависит только от класса когомологий функции $f$. Если $\delta^{*}[f]=F\left(p_{1}, p_{2}, \ldots\right), F$ - полином, то для любого комбинаторного многообразия $K$ класс гомологий $\left[f_{\sharp}(K)\right]$ двойствен по Пуанкаре классу когомологий $F\left(p_{1}(K), p_{2}(K), \ldots\right)$, где $p_{i}(K)$ - рачиональные классъ Понтрягина многообразия $K$. 
Таким образом, функция $f$ такая, что $\delta^{*}[f]=F\left(p_{1}, p_{2}, \ldots\right)$, задает универсальную локальную формулу для полинома $F$ от рациональных классов Понтрягина. Свойство универсальности заключается в том, что функция $f$ не зависит от рассматриваемого комбинаторного многообразия $K$ и коэффициент при симплексе в цикле $f_{\sharp}(K)$ зависит только от комбинаторного типа линка этого симплекса. Мы будем говорить, что такая функция $f$ является локальной формулой для полинома $F\left(p_{1}, p_{2}, \ldots\right)$. Согласно предложению 5.1 для каждого полинома от рациональных классов Понтрягина существует локальная формула, единственная с точностью до прибавления кограницы комплекса $\mathcal{T}^{*}(\mathbb{Q})$.

5.1. Локальные формулы для $L$-классов Хирцебруха. В этом пункте мы опишем явно все локальные комбинаторные формулы для $L$-полиномов Хирцебруха от классов Понтрягина. Напомним, что $L_{l}\left(p_{1}, p_{2}, \ldots, p_{l}\right)$ - однородный полином суммарной степени $4 l$ (степень переменной $p_{i}$ считается равной $4 i$ ), задаваемый по формуле

$$
1+\sum_{l=1}^{\infty} L_{l}\left(p_{1}, p_{2}, \ldots, p_{l}\right)=\prod_{j=1}^{\infty} \frac{\sqrt{t_{j}}}{\operatorname{th}\left(\sqrt{t_{j}}\right)},
$$

где $p_{i}-i$-й элементарный симметрический полином от переменных $t_{j}$. Для любого $4 l$-мерного ориентированного замкнутого многообразия $M$ имеется классическая формула Хирцебруха

$$
\operatorname{sign} M=\left\langle L_{l}\left(p_{1}(M), p_{2}(M), \ldots, p_{l}(M)\right),[M]\right\rangle .
$$

ПредложениЕ 5.2. Пустъ $f \in \mathcal{T}^{4 l}(\mathbb{Q})$ - локальная формула для l-го полинома Хирцебруха. Тогда для любого сбалансированного набора $\left(Y_{1}, Y_{2}, \ldots, Y_{k}\right)$ ориентированных $(4 l-1)$-мерных комбинаторных сфер функция $f$ удовлетворяет уравнению

$$
f\left(Y_{1}\right)+f\left(Y_{2}\right)+\cdots+f\left(Y_{k}\right)=\frac{\operatorname{sign} X}{r},
$$

где X и r-cоответственно ориентированное кубически клеточное комбинаторное многообразие и натуральное число из теоремы 1.2. Обратно, всякая функция $f \in \mathcal{T}^{4 l}(\mathbb{Q})$, удовлетворяющая системе уравнений (5.1), является локальной формулой для полинома $L_{l}$.

ДокАзАтЕльство. Пусть $f$ - локальная формула для полинома $L_{l}$. Тогда

$$
\begin{aligned}
\operatorname{sign} X & =\left\langle L_{l}\left(p_{1}(X), p_{2}(X), \ldots, p_{l}(X)\right),[X]\right\rangle=\varepsilon\left(f_{\sharp}\left(X^{\prime}\right)\right) \\
& =r\left(f\left(Y_{1}^{\prime}\right)+f\left(Y_{2}^{\prime}\right)+\cdots+f\left(Y_{k}^{\prime}\right)\right)=r\left(f\left(Y_{1}\right)+f\left(Y_{2}\right)+\cdots+f\left(Y_{k}\right)\right),
\end{aligned}
$$

где $\varepsilon: C_{0}\left(X^{\prime} ; \mathbb{Z}\right) \rightarrow \mathbb{Z}$ - аугментация. Последнее равенство вытекает из предложения 2.13 , так как функция $f$ является коциклом, а сумма $Y_{1}+Y_{2}+\cdots+Y_{k}-$ циклом.

Локальная формула для полинома $L_{l}\left(p_{1}, p_{2}, \ldots, p_{l}\right)$ определена однозначно с точностью до кограницы комплекса $\mathcal{T}^{*}(\mathbb{Q})$. С другой стороны, циклы комплекса $\mathcal{T}_{*}$ - это в точности сбалансированные наборы комбинаторных сфер. Поэтому решение $f \in \mathcal{T}^{4 l}(\mathbb{Q})$ системы уравнений (5.1) также определено однозначно с точностью до кограницы комплекса $\mathcal{T}^{*}(\mathbb{Q})$. Таким образом, решения системы (5.1) - это в точности локальные формулы для полинома $L_{l}$. 
Система уравнений (5.1) дает явное комбинаторное описание всех локальных формул для $l$-го полинома Хирцебруха, так как правые части уравнений (5.1) допускают явное комбинаторное вычисление. Действительно, кубически клеточное комбинаторное многообразие $X$ строится с помощью явной комбинаторной конструкции, описанной в $§ 2$. Его сигнатура может быть вычислена либо непосредственно по определению, либо с помощью явной (нелокальной) комбинаторной формулы, полученной А. Раницким и Д. Сулливаном в 1976 г. [24]. Поскольку $X$ - кубически клеточное комбинаторное многообразие, необходимо использовать следующую модификацию формулы Раницкого-Сулливана.

ПрЕДЛОЖЕНИЕ 5.3. Пусть $X$ - ориентированное $4 l$-мерное кубически клеточное комбинаторное многообразие. Обозначим через $C_{i}$ группу $i$-мерных клеточных цепей комплекса $X$ с фиксированным базисом, состоящим из $i$-мерных кубов комплекса $X$. Тогда

$$
\operatorname{sign} X=\operatorname{sign}\left(\begin{array}{cc}
A & B \\
B^{t} & 0
\end{array}\right),
$$

где $B$ - матрица граничного оператора $\partial: C_{2 l+1} \rightarrow C_{2 l}$ и $A-$ матрица симметрической билинейной бормы $\alpha$ на пространстве $C_{2 l}$ такой, что

$$
\alpha(\sigma, \tau)=\sum \gamma(\sigma, \tau, \eta)
$$

где суммирование ведется по всем $4 l$-мерным кубам $\eta$ комплекса $X, \gamma(\sigma, \tau, \eta)=$ \pm 1, если $\operatorname{dim}(\sigma \cap \tau)=0$ и $\sigma, \tau \subset \eta$ (знак числа $\gamma(\sigma, \tau, \eta)$ положителен, если ориентация куба $\sigma$, умноженная на ориентацию куба $\tau$, дает ориентацию куба $\eta$, совпадаюшую с заданной ориентащией многообразия $X), u \gamma(\sigma, \tau, \eta)=0$ во всех остальных случаях.

Полученное явное описание всех локальных формул для полиномов Хирцебруха является очень неэффективным. Конструкция многообразия $X$ довольно сложна, поэтому необходимо вычислять сигнатуры матриц очень большого размера. Поэтому описанные формулы вряд ли пригодны для конкретных вычислений.

5.2. Выбор канонической формулы. Кроме описания всех локальных формул для какого-либо полинома от рациональных классов Понтрягина всегда желательно уметь явно строить какую-нибудь одну каноническую локальную формулу для этого полинома. Чтобы выбрать какую-нибудь каноническую локальную формулу для полинома $L_{l}\left(p_{1}, p_{2}, \ldots, p_{l}\right)$, нам необходимо выбрать каноническое решение $f_{0}$ системы (5.1). Это может быть сделано с помощью следующего стандартного приема.

У. Пахнер в 1987 г. [37], [38] доказал, что каждая комбинаторная сфера может быть получена из границы симплекса с помощью конечного числа бизвездных преобразований (см. также [5]). Обозначим через $T_{4 l}^{(q)}$ множество ориентированных $(4 l-1)$-мерных комбинаторных сфер, которые могут быть получены из границы $4 l$-мерного симплекса с помощью последовательности из не более чем $q$ бизвездных преобразований. Заметим, что множество $T_{4 l}^{(q)}$ допускает алгоритмическое описание. Будем последовательно выбирать ограничения функции $f_{0}$ на множества $T_{4 l}^{(q)}$. Пусть уже выбрано ограничение функции $f_{0}$ 
на множество $T_{4 l}^{(q-1)}$. Из всех функций $f: T_{4 l}^{(q)} \rightarrow \mathbb{Q}$, которые совпадают на $T_{4 l}^{(q-1)}$ с уже выбранным ограничением функции $f_{0}$ и удовлетворяют уравнениям $f(-Y)=-f(Y)$ и уравнениям (5.1) для всех сбалансированных наборов комбинаторных сфер $Y_{1}, Y_{2}, \ldots, Y_{k} \in T_{4 l}^{(q)}$, в качестве ограничения функции $f_{0}$ выберем ту, для которой величина

$$
\sum_{Y \in T_{4 l}^{(q)}}(f(Y))^{2}
$$

принимает наименьшее значение. Такая функция существует и единственна, задача ее нахождения сводится к решению системы линейных уравнений и, следовательно, может быть решена алгоритмически.

ЗАмечАниЕ 5.1. Вместо множеств $T_{4 l}^{(q)}$ нельзя было бы рассматривать множества комбинаторных сфер, имеющих не более $q$ вершин, так как такие множества не допускают алгоритмического описания. Если мы работаем с симплициальными гомологическими многообразиями, то вместо множеств $T_{4 l}^{(q)}$ можно рассматривать множества симплициальных гомологических сфер, имеющих не более $q$ вершин.

ЗАмечАниЕ 5.2. Описанная в настоящем пункте процедура выбора канонического решения системы (5.1) не нужна для того, чтобы получить симплициальный цикл, класс гомологий которого двойствен по Пуанкаре $L$-классу Хирцебруха конкретного комбинаторного многообразия $K$. Действительно, чтобы получить такой цикл, нам достаточно знать значения $f(Y)$ только для тех $(4 l-1)$-мерных комбинаторных сфер $Y$, которые встречаются среди линков симплексов многообразия $K$. Поэтому мы должны рассмотреть только те уравнения (5.1), которые соответствуют сбалансированным наборам $\left(Y_{1}, Y_{2}, \ldots, Y_{k}\right)$ таким, что каждая из комбинаторных сфер $Y_{i}$ изоморфна линку некоторого симплекса многообразия $K$. Среди таких уравнений есть лишь конечное число линейно независимых. Таким образом, мы получаем конечную систему линейных уравнений, и нам достаточно взять произвольное ее решение.

5.3. Умножение локальных формул. Согласно следствию 3.4 имеется аддитивный изоморфизм

$$
\delta^{*}: H^{*}\left(\mathcal{T}^{*}(\mathbb{Q})\right) \rightarrow \operatorname{Hom}\left(\Omega_{*}^{\mathrm{SPL}}, \mathbb{Q}\right) \cong \mathbb{Q}\left[p_{1}, p_{2}, \ldots\right] .
$$

Естественно возникает вопрос о том, как описать каким-либо явным комбинаторным способом умножение, возникающее в когомологиях комплекса $\mathcal{T}^{*}(\mathbb{Q})$ (см. [2]). В этом параграфе мы комбинаторно определим операцию умножения на коциклах комплекса $\mathcal{T}^{*}(\mathbb{Q})$, которая индуцирует искомое умножение в когомологиях. K сожалению, эта операция умножения не является ни билинейной, ни ассоциативной, ни коммутативной, не удовлетворяет тождеству Лейбница и, по-видимому, не имеет естественного продолжения на весь комплекс $\mathcal{T}^{*}(\mathbb{Q})$. Таким образом, следующий вопрос остается открытым.

Вопрос 5.1. Существует ли в комплексе $\mathcal{T}^{*}(\mathbb{Q})$ билинейное ассоциативное умножение, удовлетворяющее формуле Лейбница и индуцирующее в когомологиях умножение, относительно которого изоморфизм $\delta^{*}$ является мультипликативным? 
Комбинаторное определение даже небилинейного и неассоциативного умножения коциклов комплекса $\mathcal{T}^{*}(\mathbb{Q})$ сразу дает нам возможность по двум заданным локальным формулам для двух полиномов от рациональных классов Понтрягина построить явно локальную формулу для произведения этих полиномов. Таким образом, в сочетании с явными локальными формулами для $L$-полиномов Хирцебруха, построенными в пп. 5.1, 5.2, этот результат дает нам явные локальные формулы для всех полиномов от рациональных классов Понтрягина. (Напомним, что полиномы Хирцебруха порождают кольцо $\left.\mathbb{Q}\left[p_{1}, p_{2}, \ldots\right].\right)$

Коциклы коцепного комплекса $\mathcal{T}^{*}(\mathbb{Q})$ задают локальные формулы для циклов, классы гомологии которых двойственны по Пуанкаре полиномам от классов Понтрягина. Однако когда мы хотим по локальным формулам для двух полиномов от классов Понтрягина построить локальную формулу для их произведения, нам удобнее работать с коциклами, представляющими полиномы от классов Понтрягина. Впервые локальные формулы для коциклов, представляющих характеристические классы, рассматривались Н. Левиттом и К. Рурком [1]. При этом рассматривались симплициальные коциклы в первом барицентрическом подразделении данного комбинаторного многообразия, значения которых на каждом симплексе зависят только от комбинаторного строения звезды младшей вершины этого симплекса. (Младиая вериина - вершина, являющаяся барицентром симплекса наименьшей размерности.) Нам будет удобнее работать не с барицентрическим подразделением, а с каноническим кубическим подразделением комбинаторного многообразия (см. далее п. 5.5). В следующем пункте мы определяем коцепной комплекс $\mathcal{W}^{*}(A)$, являющийся аналогом комплекса $\mathcal{T}^{*}(A)$ в рассматриваемой ситуации. При этом для любого кольца $\Lambda$ в комплексе $\mathcal{W}^{*}(\Lambda)$ вводится ассоциативное умножение, удовлетворяющее формуле Лейбница. Основным результатом является теорема 5.1, устанавливающая изоморфизм $H^{*}\left(\mathcal{T}^{*}(\mathbb{Q})\right) \cong H^{*}\left(\mathcal{W}^{*}(\mathbb{Q})\right)$. Эта теорема дает возможность использовать умножение в комплексе $\mathcal{W}^{*}(\mathbb{Q})$ для построения искомого умножения коциклов комплекса $\mathcal{T}^{*}(\mathbb{Q})$ (см. далее п. 5.7).

5.4. Коцепной комплекс $\mathcal{W}^{*}(A)$. Как было отмечено в п. 2.1, имеются два эквивалентных определения линков симплексов симплициального комплекса. В оставшейся части данной статьи нам будет удобно использовать не то определение, которое было принято ранее. Таким образом, с настоящего момента мы будем определять линк симплекса $\sigma$ симплициального комплекса $K$ как подкомплекс комплекса $K$, состоящий из всех симплексов $\tau$ таких, что $\sigma \cap \tau=\varnothing$ и в комплексе $K$ имеется симплекс, содержащий и симплекс $\sigma$, и симплекс $\tau$.

Для каждой абелевой группы $A$ определим коцепной комплекс $\mathcal{W}^{*}(A)$ следующим образом. Элементами группы $\mathcal{W}^{n}(A)$ будут функции $h$ такие, что $h$ сопоставляет каждой комбинаторной сфере $Y$ (произвольной размерности и без выделенной ориентации) симплициальную коцепь $h(Y) \in C^{n-1}(Y ; A)$, так что выполнено следующее условие: если $i: Y_{1} \rightarrow Y_{2}$ - изоморфизм комбинаторных сфер, то индуцированное отображение коцепей переводит коцепь $h\left(Y_{2}\right)$ в коцепь $h\left(Y_{1}\right)$. В частности, коцепь $h(Y)$ должна быть инвариантна относительно всех (в том числе, меняющих ориентацию) автоморфизмов комбинаторной сферы $Y$. Значение коцепи $h(Y)$ на цепи $\xi \in C_{n-1}(Y ; \mathbb{Z})$ мы будем обозначать 
через $h(Y, \xi)$. Как и раньше, мы часто не будем различать комбинаторную сферу и ее класс изоморфизма. Определим отображение

$$
\delta: \mathcal{W}^{n}(A) \rightarrow \mathcal{W}^{n+1}(A)
$$

по формуле

$$
(\delta h)(Y, \xi)=(-1)^{n} h(Y, \partial \xi)+(-1)^{n-1} \sum_{y \in \operatorname{Vert}(Y)} h\left(\operatorname{link} y, \xi_{y}\right),
$$

где $\xi_{y}$ - цепь, в которую каждый симплекс $\sigma$ комплекса link $y$ входит с коэффициентом, равным коэффициенту при симплексе $y * \sigma$ в цепи $\xi$.

ПРЕДЛОЖЕНИЕ 5.4. Имеем $\delta^{2}=0$.

ДокАзАТЕЛЬСтво. Введем обозначения

$$
\left(\delta_{1} h\right)(Y, \xi)=(-1)^{n} h(Y, \partial \xi), \quad\left(\delta_{2} h\right)(Y, \xi)=(-1)^{n-1} \sum_{y \in \operatorname{Vert}(Y)} h\left(\operatorname{link} y, \xi_{y}\right) .
$$

Очевидно, что $\delta_{1}^{2}=0$. Для доказательства равенства $\delta_{2}^{2}=0$ достаточно заметить, что $\left(\xi_{x}\right)_{y}=-\left(\xi_{y}\right)_{x}$ для любой цепи $\xi \in C_{n-1}(Y ; \mathbb{Z})$ и любых двух вершин $x, y \in Y$, соединенных ребром. Для доказательства равенства $\delta_{1} \delta_{2}+\delta_{2} \delta_{1}=0$ достаточно заметить, что $\partial\left(\xi_{y}\right)=-(\partial \xi)_{y}$ для любой цепи $\xi \in C_{n-1}(Y ; \mathbb{Z})$ и любой вершины $y \in Y$.

Таким образом, $\mathcal{W}^{*}(A)$ - коцепной комплекс с дифференциалом $\delta$.

Определим отображение $\alpha: \mathcal{W}^{n}(A) \rightarrow \mathcal{T}^{n}(A)$ по формуле

$$
\alpha(h)(Y)=h(Y,[Y]),
$$

где $[Y]$ - фундаментальный цикл комбинаторной сферы $Y$. Из того, что коцепь $h(Y)$ не зависит от выбора ориентации комбинаторной сферы $Y$, следует, что значение $\alpha(h)(Y)$ меняет знак при изменении ориентации комбинаторной сферы $Y$. Несложно проверить, что $\alpha$ - цепное отображение. Следовательно, оно индуцирует гомоморфизм

$$
\alpha^{*}: H^{*}\left(\mathcal{W}^{*}(A)\right) \rightarrow H^{*}\left(\mathcal{T}^{*}(A)\right) .
$$

Теорема 5.1. При $A=\mathbb{Q}$ гомоморфизм $\alpha^{*}$ является изоморфизмом.

Доказательство теоремы 5.1 мы проведем далее, в п. 5.8.

Пусть $Y$ - комбинаторная сфера, $\xi \in C_{l}(Y ; \mathbb{Z})$ - симплициальная цепь, $\tau$ ориентированный $(n-1)$-мерный симплекс комплекса $Y$. Обозначим через $\xi_{\tau} \in C_{l-n}(\operatorname{link} \tau ; \mathbb{Z})$ цепь, в которую каждый симплекс $\rho$ комплекса $\operatorname{link} \tau$ входит с коэффициентом, равным коэффициенту при симплексе $\tau * \rho$ в цепи $\xi$.

Пусть $\Lambda$ - ассоциативное кольцо. Определим умножение

$$
\mathcal{W}^{n}(\Lambda) \otimes \mathcal{W}^{k}(\Lambda) \rightarrow \mathcal{W}^{n+k}(\Lambda)
$$

по формуле

$$
\left(h_{1} h_{2}\right)(Y, \xi)=(-1)^{n k} \sum_{\substack{\tau-\text { симплекс } Y \\ \operatorname{dim} \tau=n-1}} h_{1}(Y, \tau) h_{2}\left(\operatorname{link} \tau, \xi_{\tau}\right),
$$


где $\xi \in C_{n+k-1}(Y ; \mathbb{Z})$. (Слагаемое $h_{1}(Y, \tau) h_{2}\left(\operatorname{link} \tau, \xi_{\tau}\right)$ не зависит от выбора ориентации симплекса $\tau$.) Несложно проверить, что введенное умножение является ассоциативным. Непосредственно проверяется, что

$$
\delta\left(h_{1} h_{2}\right)=\left(\delta h_{1}\right) h_{2}+(-1)^{n} h_{1} \delta h_{2}
$$

для любых $h_{1} \in \mathcal{W}^{n}(\Lambda), h_{2} \in \mathcal{W}^{k}(\Lambda)$. Следовательно, построенное умножение индуцирует ассоциативное умножение в когомологиях комплекса $\mathcal{W}^{*}(\Lambda)$.

\section{5. Каноническое кубическое подразделение симплициального} комплекса. Для каждого симплициального комплекса $K$ определено его каноническое кубическое подразделение $\operatorname{cub}(K)$ (см., например, [5]). Опишем здесь эту конструкцию. Пусть комплекс $K$ имеет $q$ вершин. Будем отождествлять множество вершин комплекса $K$ с множеством $\{1,2, \ldots, q\}$. Пусть $I^{q}=[0,1]^{q}-$ стандартный куб. Для любых непустых симплексов $\tau \subset \sigma$ комплекса $K$ положим

$$
C_{\tau \subset \sigma}=\left\{\left(y_{1}, y_{2}, \ldots, y_{q}\right) \in I^{q}: y_{j}=0 \text { при } j \in \tau, \quad y_{j}=1 \text { при } j \notin \sigma\right\} .
$$

Тогда $C_{\tau \subset \sigma}-$ замкнутая грань куба $I^{q}$ размерности $\operatorname{dim} \sigma-\operatorname{dim} \tau$. Пусть $i: K \rightarrow I^{q}$ - отображение, переводящее барицентр каждого симплекса $\sigma$ комплекса $K$ в вершину $C_{\sigma \subset \sigma}$ и линейное на всех симплексах барицентрического подразделения комплекса $K$. Тогда $i$ - вложение, образ которого совпадает с объединением всех граней $C_{\tau \subset \sigma}$, где $\tau \subset \sigma$ - симплексы $K$. Прообразы граней $C_{\tau \subset \sigma}$ при отображении $i$ (которые мы будем также обозначать через $C_{\tau \subset \sigma}$ ) образуют кубическое подразделение $\operatorname{cub}(K)$ симплициального комплекса $K$.

Если симплексы $\tau$ и $\sigma$ ориентированы, то на клетке $C_{\tau \subset \sigma}$ выбирается ориентация такая, что произведение ориентации симплекса $\tau$ на ориентацию клетки $C_{\tau \subset \sigma}$ дает ориентацию симплекса $\sigma$. Определим умножение в клеточных коцепях $C^{*}(\operatorname{cub}(K) ; \Lambda)$ разбиения $\operatorname{cub}(K)$ по формуле

$$
(a b)\left(C_{\tau \subset \sigma}\right)=(-1)^{n k} \sum_{\substack{\rho-\text { симплекс } K \\ \operatorname{dim} \rho=\operatorname{dim} \tau+n}} a\left(C_{\tau \subset \rho}\right) b\left(C_{\rho \subset \sigma}\right),
$$

где $a \in C^{n}(\operatorname{cub}(K) ; \Lambda), b \in C^{k}(\operatorname{cub}(K) ; \Lambda), \sigma$ и $\tau$ - ориентированные симплексы комплекса $K$ такие, что $\operatorname{dim} \sigma-\operatorname{dim} \tau=n+k$.

ПРЕДЛОЖЕНИЕ 5.5. Введенное умножение ассоииативно, удовлетворяет формуле Лейбница и индуиирует стандартное умножение в когомологиях пространства $K$.

5.6. Локальные формулы для коциклов. Пусть $K$ - комбинаторное многообразие, $h$ - произвольный элемент группы $\mathcal{W}^{n}(A)$. Определим коцепь

$$
h^{\sharp}(K) \in C^{n}(\operatorname{cub}(K) ; A)
$$

по формуле

$$
h^{\sharp}(K)\left(C_{\tau \subset \sigma}\right)=h\left(\operatorname{link} \tau, \sigma_{\tau}\right) .
$$

Следующие предложения проверяются непосредственно. 
ПРЕДЛОЖЕНИЕ 5.6. Имеем $\delta\left(h^{\sharp}(K)\right)=(\delta h)^{\sharp}(K)$.

СлеДСтвиЕ 5.1. Коцепь $h^{\sharp}(K)$ является коциклом для любого комбинаторного многообразия $K$ тогда и только тогда, когда $h$ является коииклом в комплексе $\mathcal{W}^{*}(A)$. Если $h$ является кограницей в комплексе $\mathcal{W}^{*}(A)$, то коцепь $h^{\sharp}(K)$ является когранищей для любого комбинаторного многообразия $K$.

ПреДЛОЖЕНИЕ 5.7. Пусть $\Lambda-$ кольцо, $h_{1}, h_{2} \in \mathcal{W}^{*}(\Lambda)$. Тогда

$$
\left(h_{1} h_{2}\right)^{\sharp}(K)=h_{1}^{\sharp}(K) h_{2}^{\sharp}(K)
$$

для любого комбинаторного многообразия $K$.

ПредлОЖенИЕ 5.8. Пусть $h \in \mathcal{W}^{n}(A)-\kappa о ц и к л, f=\alpha(h)$. Тогда для любого ориентированного комбинаторного многообразия $K$ класс гомологий, представляемый ииклом $f_{\sharp}(K)$, двойствен по Пуанкаре классу когомологий, представляемому коциклом $h^{\sharp}(K)$.

ДокАЗАТЕЛЬСтво. Обозначим через $m$ размерность многообразия $K$. Пусть $\tau$ - симплекс размерности $m-n$. Разбиение $\mathrm{cub}(K)$ является подразделением разбиения $K^{*}$, двойственного триангуляции $K$. При этом $n$-мерная клетка $D \tau$, двойственная симплексу $\tau$, является объединением кубов $C_{\tau \subset \sigma}$, где $\sigma-$ всевозможные $m$-мерные симплексы, содержащие $\tau$. Пусть $c$ - образ коцепи $h^{\sharp}(K)$ при естественном отображении $C^{n}(\operatorname{cub}(K) ; A) \rightarrow C^{n}\left(K^{*} ; A\right)$. Тогда

$$
c(D \tau)=\sum_{\substack{\sigma \supset \tau \\ \operatorname{dim} \sigma=m}} h^{\sharp}(K)\left(C_{\tau \subset \sigma}\right)=h(\operatorname{link} \tau,[\operatorname{link} \tau])=f(\operatorname{link} \tau) .
$$

Следовательно, коцикл $c$ двойствен по Пуанкаре циклу $f_{\sharp}(K)$.

Рассмотрим изоморфизмы

$$
H^{*}\left(\mathcal{W}^{*}(\mathbb{Q})\right) \stackrel{\alpha^{*}}{\longrightarrow} H^{*}\left(\mathcal{T}^{*}(\mathbb{Q})\right) \stackrel{\delta^{*}}{\longrightarrow} \Omega_{*}^{\mathrm{SPL}} \otimes \mathbb{Q}=\mathbb{Q}\left[p_{1}, p_{2}, \ldots\right] .
$$

Пусть $h \in \mathcal{W}^{*}(\mathbb{Q})$ - коцикл, $F\left(p_{1}, p_{2}, \ldots\right)=\delta^{*} \alpha^{*}[h]-$ соответствующий полином от рациональных классов Понтрягина. Из предложения 5.8 сразу следует, что коцикл $h^{\sharp}(K)$ представляет класс когомологий $F\left(p_{1}(K), p_{2}(K), \ldots\right)$ для любого комбинаторного многообразия $K$. В частности, класс когомологий $\left[h^{\sharp}(K)\right]$ не зависит от выбора коцикла $h$, представляющего класс когомологий $[h]$, и кусочно линейной триангуляции $K$ заданного многообразия. Мы будем говорить, что коцикл $h$ является локальной формулой для полинома $F$ от классов Понтрягина. Из теоремы 5.1 следует, что для любого полинома от рациональных классов Понтрягина существует локальная формула $h \in \mathcal{W}^{*}(\mathbb{Q})$, единственная с точностью до прибавления кограницы комплекса $\mathcal{W}^{*}(\mathbb{Q})$.

5.7. Произведение коциклов комплекса $\mathcal{T}^{*}(\mathbb{Q})$. Обозначим через $Z^{n}$ подгруппу группы $\mathcal{T}^{n}(\mathbb{Q})$, состоящую из всех коциклов, т. е. локальных формул для полиномов от классов Понтрягина (см. предложение 5.1). Построим явно отображение $\gamma: Z^{n} \rightarrow \mathcal{W}^{n}(\mathbb{Q})$ такое, что $\gamma(f)-$ коцикл для любой локальной формулы $f$ и $\alpha \circ \gamma$ - тождественное отображение.

Пусть $f \in Z^{n}$ - коцикл. Коцепь $h=\gamma(f)$ должна удовлетворять условиям $\delta h=0$ и $\alpha(h)=f$, т. е. системе уравнений двух типов: 
1) $h(Y, \partial \xi)=\sum_{y \in \operatorname{Vert}(Y)} h\left(\operatorname{link} y, \xi_{y}\right)$ для любой комбинаторной сферы $Y$ и любой цепи $\xi \in C_{n}(Y ; \mathbb{Z})$;

2) $h(Y,[Y])=f(Y)$ для любой ориентированной $(n-1)$-мерной комбинаторной сферы $Y$.

Мы будем строить набор коцепей $h(Y)$, удовлетворяющий этой системе уравнений, применяя индукцию по размерности комбинаторной сферы $Y$.

Для каждой ориентированной $(n-1)$-мерной комбинаторной сферы $Y$, имеющей $q$ симплексов старшей размерности, положим

$$
h(Y, \sigma)=\frac{f(Y)}{q}
$$

для любого положительно ориентированного $(n-1)$-мерного симплекса $\sigma$ комплекса $Y$. Очевидно, что коцепь $h(Y)$ не меняется при изменении ориентации комбинаторной сферы $Y$.

Пусть $m \geqslant n$. Предположим, что коцепи $h(Y)$ уже определены для всех комбинаторных сфер $Y$, размерность которых меньше $m$. Определим коцепь $h(Y)$ для $m$-мерной комбинаторной сферы $Y$. Пусть $\sigma_{1}, \sigma_{2}, \ldots, \sigma_{q}-$ все $(n-1)$-мерные симплексы комбинаторной сферы $Y$, для каждого из которых фиксирована какая-нибудь ориентация. Рассмотрим всевозможные уравнения вида

$$
h(Y, \partial \xi)=\sum_{y \in \operatorname{Vert}(Y)} h\left(\operatorname{link} y, \xi_{y}\right),
$$

где $\xi \in C_{n}(Y ; \mathbb{Z})$. Каждое из этих уравнений можно рассматривать как линейное уравнение с неизвестными $h\left(Y, \sigma_{1}\right), h\left(Y, \sigma_{2}\right), \ldots, h\left(Y, \sigma_{q}\right)$.

ПРЕДЛОЖЕНИЕ 5.9. Система линейных уравнений (5.2) совместна.

ДоказАтельство. Достаточно доказать, что

$$
\sum_{y \in \operatorname{Vert}(Y)} h\left(\operatorname{link} y, \xi_{y}\right)=0
$$

если $\xi$ - цикл. Рассмотрим два случая.

Если $m=n$, то $\xi=l[Y]$ для некоторого $l \in \mathbb{Z}$. Тогда

$$
\sum_{y \in \operatorname{Vert}(Y)} h\left(\operatorname{link} y, \xi_{y}\right)=l \sum_{y \in \operatorname{Vert}(Y)} f(\operatorname{link} y)=(-1)^{n} l(\delta f)(Y)=0 .
$$

Если $m>n$, то $\xi=\partial \eta$ для некоторой цепи $\eta \in C_{n+1}(Y ; \mathbb{Z})$. Значит,

$$
\begin{aligned}
\sum_{y \in \operatorname{Vert}(Y)} h\left(\operatorname{link} y, \xi_{y}\right) & =\sum_{y \in \operatorname{Vert}(Y)} h\left(\operatorname{link} y,(\partial \eta)_{y}\right)=-\sum_{y \in \operatorname{Vert}(Y)} h\left(\operatorname{link} y, \partial\left(\eta_{y}\right)\right) \\
& =\sum_{(x, y)} h\left(\operatorname{link}(x * y),\left(\eta_{y}\right)_{x}\right)=0,
\end{aligned}
$$

где последняя сумма берется по всем парам вершин $(x, y)$, соединенных ребром в комплексе $Y$. Последнее равенство следует из того, что $\left(\eta_{x}\right)_{y}=-\left(\eta_{y}\right)_{x}$. 
В качестве коцепи $h(Y)$ выберем то решение системы линейных уравнений (5.2), для которого сумма

$$
h\left(Y, \sigma_{1}\right)^{2}+h\left(Y, \sigma_{2}\right)^{2}+\cdots+h\left(Y, \sigma_{q}\right)^{2}
$$

принимает наименьшее значение. Такое решение существует, единственно, рационально, его вычисление сводится к решению системы линейных уравнений.

Заметим, что для вычисления коцепи $h(Y)$, где $\operatorname{dim} Y=m$, достаточно знать только значения функции $f$ на линках всех $(m-n)$-мерных симплексов комбинаторной сферы $Y$.

Определим операцию умножения $Z^{n} \times Z^{k} \rightarrow Z^{n+k}$ по формуле

$$
f_{1} \diamond f_{2}=\alpha\left(\gamma\left(f_{1}\right) \gamma\left(f_{2}\right)\right)
$$

Это умножение не будет ни ассоциативным, ни коммутативным, ни линейным по первому аргументу. Можно проверить, что оно будет линейным по второму аргументу. Из предложений 5.7 и 5.8 немедленно следует, что если $f_{1}$ и $f_{2}$ - локальные формулы для полиномов $F_{1}\left(p_{1}, p_{2}, \ldots\right)$ и $F_{2}\left(p_{1}, p_{2}, \ldots\right)$ соответственно, то $f_{1} \diamond f_{2}$ - локальная формула для полинома $F_{1}\left(p_{1}, p_{2}, \ldots\right) F_{2}\left(p_{1}, p_{2}, \ldots\right)$. Таким образом, умножение $\diamond$ индуцирует в когомологиях комплекса $\mathcal{T}^{*}(\mathbb{Q})$ умножение, совпадающее с умножением, определенным с помощью изоморфизма $\delta^{*}$.

ЗАмЕчАниЕ 5.3. Для вычисления значения функции $f_{1} \diamond f_{2}$ на $(n+k-1)$ мерной комбинаторной сфере $Y$ нам достаточно знать только значения функции $f_{1}$ на линках всех $(k-1)$-мерных симплексов комбинаторной сферы $Y$ и значения функции $f_{2}$ на линках всех $(n-1)$-мерных симплексов комбинаторной сферы $Y$. При этом процедура вычисления значения $\left(f_{1} \diamond f_{2}\right)(Y)$ сводится к решению систем линейных уравнений. Таким образом, согласно замечанию 5.2 для вычисления цикла, класс гомологий которого двойствен по Пуанкаре данному полиному от рациональных классов Понтрягина данного комбинаторного многообразия $K$, нам не нужно использовать процедуру выбора канонических локальных формул для $L$-классов Хирцебруха, описанную в п. 5.2. В процессе вычисления нам придется оперировать лишь с теми комбинаторными сферами, которые встречаются среди линков симплексов многообразия $K$.

5.8. Доказательство теоремы 5.1. Эпиморфность отображения $\alpha^{*}$ сразу следует из того, что для каждого коцикла $f \in \mathcal{T}^{n}(\mathbb{Q})$ существует коцикл $h=\gamma(f) \in \mathcal{W}^{n}(\mathbb{Q})$ такой, что $\alpha(h)=f$.

Пусть $h \in \mathcal{W}^{n}(\mathbb{Q})$ - коцикл, $\alpha(h)=\delta f$. Тогда $\alpha(h-\delta \gamma(f))=0$. Поэтому для доказательства мономорфности отображения $\alpha^{*}$ достаточно доказать, что если $\alpha(h)=0$, то существует элемент $g \in \mathcal{W}^{n-1}(\mathbb{Q})$ такой, что $h=\delta g$. Условие $h=\delta g$ можно переписать в виде системы уравнений

$$
g(Y, \partial \xi)=\sum_{y \in \operatorname{Vert}(Y)} g\left(\operatorname{link} y, \xi_{y}\right)+(-1)^{n-1} h(Y, \xi),
$$

где $Y$ - произвольная комбинаторная сфера и $\xi \in C_{n-1}(Y ; \mathbb{Z})-$ произвольная цепь.

Будем последовательно определять коцепи $g(Y)$. Для любой $(n-2)$-мерной комбинаторной сферы $Y$ возьмем $g(Y)=0$. 
Пусть $m \geqslant n-1$. Предположим, что коцепи $g(Y)$ уже определены для всех комбинаторных сфер размерности меньше $m$. Определим коцепь $g(Y)$ для $m$-мерной комбинаторной сферы $Y$. Пусть $\sigma_{1}, \sigma_{2}, \ldots, \sigma_{q}$ - все $(n-2)$-мерные симплексы комбинаторной сферы $Y$, для каждого из которых фиксирована какая-нибудь ориентация. Рассмотрим всевозможные уравнения вида (5.3), где $\xi \in C_{n-1}(Y ; \mathbb{Z})$. Каждое из этих уравнений можно рассматривать как линейное уравнение с неизвестными $g\left(Y, \sigma_{1}\right), g\left(Y, \sigma_{2}\right), \ldots, g\left(Y, \sigma_{q}\right)$.

ПРЕДЛОЖЕНИЕ 5.10. Рассматриваемая система линейных уравнений совместна.

ДокАЗАТЕЛЬСТво аналогично доказательству предложения 5.9. В качестве коцепи $g(Y)$ выберем произвольное решение рассматриваемой системы линейных уравнений, инвариантное относительно всех автоморфизмов комбинаторной сферы $Y$.

\section{Список литературы}

1. N. Levitt, C. Rourke, "The existence of combinatorial formulae for characteristic classes", Trans. Amer. Math. Soc., 239 (1978), 391-397.

2. А.А. Гайфуллин, "Локальные формулы для комбинаторных классов Понтрягина", Изв. РАН. Сер. матем., 68:5 (2004), 13-66; англ. пер.: A. A. Gaifullin, "Local formulae for combinatorial Pontryagin classes", Izv. Math., 68:5 (2004), 861-910.

3. А.А. Гайфуллин, "Вычисление характеристических классов многообразия по его триангуляции", УМН, 60:4 (2005), 37-66; англ. пер.: A. A. Gaifullin, "Computation of characteristic classes of a manifold from a triangulation of it", Russian Math. Surveys, 60:4 (2005), 615-644.

4. M. W. Davis, T. Januszkiewicz, "Convex polytopes, Coxeter orbifolds and torus actions", Duke Math. J., 62:2 (1991), 417-451.

5. В. М. Бухштабер, Т. Е. Панов, Торические действия в топологии и комбинаторике, МЦНМО, М., 2004.

6. V. M. Buchstaber, A. P. Veselov, "On a remarkable functional equation in the theory of generalized Dunkl operators and transformations of elliptic genera", Math. Z., 223:4 (1996), 595-607.

7. В. М. Бухштабер, С. П. Новиков, "Формальные группы, степенные системы и операторы Адамса”, Матем. сб., 84:1 (1971), 81-118; англ. пер.: V. M. Bukhshtaber, S.P. Novikov, "Formal groups, power systems and Adams operators", Math. USSR-Sb., 13:1 (1971), 80-116.

8. D. Sullivan, "On the Hauptvermutung for manifolds", Bull. Amer. Math. Soc., 73:4 (1967), 598-600.

9. Д. Сулливан, Геометрическая топология, ИО НФМИ, Новокузнецк, 1999; пер. с англ.: D. Sullivan, Geometric topology. Part I. Localization, periodicity, and Galois symmetry, Massachusetts Institute of Technology, Cambridge, MA, 1971.

10. N. A. Baas, "On bordism theory of manifolds with singularities", Math. Scand., 33:2 (1973), 279-302.

11. D. Sullivan, "Singularities in spaces", Proc. of Liverpool Singularities Symposium II, Lecture notes in Math., 209, Springer, Berlin, 1971, 196-206.

12. Р. Том, "Некоторые свойства "в целом" дифференцируемых многообразий", Рacслоенные пространства, ИЛ, М., 1958, 291-348.

13. M. Kato, "Topological resolution of singularities", Topology, 12:4 (1973), 355-372. 
14. В. М. Бухштабер, "Модули дифференциалов спектральной последовательности Атья-Хирцебруха", Матем. сб., 78:2 (1969), 307-320; англ. пер.: V. M. Buchstaber, "Modules of differentials of the Atiyah-Hirzebruch spectral sequence", Math. USSR Sb., 7:2 (1969), 299-313; В. М. Бухштабер, "Модули дифференциалов спектральной последовательности Атья-Хирцебруха. II", Матем. сб., 83:1 (1970), 61-76; англ. пер.: V.M. Buchstaber, "Modules of differentials of the Atiyah-Hirzebruch spectral sequence. II", Math. USSR Sb., 12:1 (1970), 59-75.

15. S. Buoncristiano, M. Dedò, "On resolving singularities and relating bordisms to homology", Ann. Scuola Norm. Sup. Pisa Cl. Sci. (4), 7:4 (1980), 605-624.

16. А. М. Габриэлов, И. М. Гельфанд, М. В. Лосик, "Комбинаторное вычисление характеристических классов", Функи. анализ и его прил., 9:2 (1975), 12-28; англ. пер.: A. M. Gabriélov, I. M. Gel'fand, M.V. Losik, "Combinatorial computation of characteristic classes", Funct. Anal. Appl., 9:2 (1975), 103-115; А. М. Габриэлов, И. М. Гельфанд, М.В. Лосик, "Комбинаторное вычисление характеристических классов. II", Функи. анализ и его прил., 9:3 (1975), 5-26; англ. пер.: A. M. Gabriélov, I. M. Gel'fand, M. V. Losik, "Combinatorial calculus of characteristic classes", Funct. Anal. Appl., 9:3 (1975), 186-202.

17. R. MacPherson, "The combinatorial formula of Gabrielov, Gelfand and Losik for the first Pontrjagin class", Sèminaire Bourbaki, Lecture Notes in Math., 677, Springer, Berlin, 1978, 105-124.

18. I. M. Gelfand, R. D. MacPherson, "A combinatorial formula for the Pontrjagin classes", Bull. Amer. Math. Soc. (N.S.), 26:2 (1992), 304-309.

19. J. Cheeger, "Spectral geometry of singular Riemannian spaces", J. Differential Geom., 18:4 (1983), 575-657.

20. А. С. Мищенко, “Локальная комбинаторная формула Хирцебруха", Тр. МИАН, 244, Наука, M., 1999, 249-263; англ. пер.: A. S. Mishchinko, "Local combinatorical Hirzebruch formula", Proc. Steklov Inst. Math., 224:1 (1999), 226-239.

21. В. А. Рохлин, А. С. Шварц, "О комбинаторной инвариантности классов Понтрягина", Докл. АН СССР, 114:3 (1957), 490-493.

22. R. Thom, "Les classes charactéristiques de Pontrjagin des variétés triangulées", International symposium on algebraic topology, Universidad Nacional Autonoma de Mexico and UNESCO, Mexico, 1958, 54-67.

23. Дж. Милнор, Дж. Сташеф, Характеристические классы, Мир, М., 1979; пер. с англ.: J. W. Milnor, J.D. Stasheff, Characteristic classes, Annals of Mathematics Studies, 76, Princeton University Press, Princeton, NJ; University of Tokyo Press, Tokyo, 1974.

24. A. Ranicki, D. Sullivan, "A semi-local combinatorial formula for the signature of a 4k-manifold", J. Differential Geom., 11:1 (1976), 23-29.

25. M. Goresky, R. MacPherson, "Intersection homology theory", Topology, 19:2 (1980), $135-162$.

26. M. Pezzana, "Diagrammi di Heegaard e triangolazione contratta", Boll. Un. Mat. Ital. (4), 12:3 (1975), 98-105.

27. M. Ferri, "Una rappresentazione delle $n$-varietà topologiche triangolabili mediante grafi $(n+1)$-colorati", Boll. Un. Mat. Ital. B (5), 13:1 (1976), 250-260.

28. M. Ferri, C. Gagliardi, L. Grasselli, "A graph-theoretical representation of PL-manifolds - A survey on crystallizations", Aequationes Math., 31:2-3 (1986), $121-141$.

29. Б. А. Дубровин, С. П. Новиков, А. Т. Фоменко, Современная геометрия: Методъ и приложения. Т. 3: Теория гомологий, Эдиториал УРСС, М., 2001.

30. J. Cheeger, "On the Hodge theory of Riemannian pseudomanifolds", Geometry of the Laplace operator. Proc. Sympos. Pure Math. (Univ. Hawaii, Honolulu, 1979), Proc. Sympos. Pure Math., XXXVI, Amer. Math. Soc., Providence, RI, 1980, 91-145. 
31. М. Горески, Р. Мак-Ферсон, Дж. Чигер, " $\mathcal{L}^{2}$-когомологии и ГМ-гомологии особых алгебраических многообразий”, Добавление к кн., У. Фултон, Р. Мак-Ферсон, Kaтегорный подход $к$ изучению пространств с особенностями. Математика: новое в зарубежной науке, 33, Мир, М., 1983.

32. C. R.F. Maunder, "On the Pontrjagin classes of homology manifolds", Topology, 10:2 (1971), 111-118.

33. N. Martin, "On the difference between homology and piecewise-linear bundles", J. London Math. Soc. (2), 6:2 (1973), 197-204.

34. M. Furuta, "Homology cobordism group of homology 3-spheres", Invent. Math., 100:1 (1990), 339-355.

35. П. Коннер, Э. Флойд, Гладкие периодические отображения, Мир, М., 1969; пер. с англ.: P.E. Conner, E.E. Fluyd, Differentiable periodic maps, Ergebnisse der Mathematik und ihrer Grenzgebiete, 33, Academic Press, New York; Springer-Verlag, Berlin-Göttingen-Heidelberg, 1964.

36. В. М. Бухштабер, "Характеристические классы в кобордизмах и топологические приложения теорий однозначных и двузначных формальных групп", Итоги науки и техн. Соврем. пробл. математики, 10, ВИНИТИ, М., 1978, 5-178.

37. U. Pachner, "Konstruktionsmethoden und das kombinatorische Homöomorphieproblem für Triangulationen kompakter semilinearer Mannigfaltigkeiten", Abh. Math. Sem. Univ. Hamburg, 57 (1987), 69-86.

38. U. Pachner, "P. L. homeomorphic manifolds are equivalent by elementary shellings", European J. Combin., 12:2 (1991), 129-145.

A. А. ГАйфУллин (А. А. Gaifullin)

Поступило в редакцию

Московский государственный университет

20.06.2007

им. М. В. Ломоносова

E-mail: gaifull@mccme.ru 